\title{
DEVELOPING HUMAN FACTORS METRICS AND TOOLS TO SUPPORT DESIGN AND MANAGEMENT OF PRODUCTION
}

\author{
by \\ Michael Alexander Greig \\ M.Sc. Kinesiology, University of Waterloo, 2001 \\ B.Sc. Hons Kinesiology, University of Western Ontario, 1998
}

\author{
A dissertation \\ presented to Ryerson University \\ in partial fulfillment of the \\ requirements for the degree of \\ Doctor of Philosophy \\ in the Program of
}

Mechanical and Industrial Engineering

Toronto, Ontario, Canada, 2016

(C)Michael Greig 2016 


\section{AUTHOR'S DECLARATION FOR ELECTRONIC SUBMISSION OF A DISSERTATION}

I hereby declare that I am the sole author of this dissertation. This is a true copy of the dissertation, including any required final revisions, as accepted by my examiners.

I authorize Ryerson University to lend this dissertation to other institutions or individuals for the purpose of scholarly research.

I further authorize Ryerson University to reproduce this dissertation by photocopying or by other means, in total or in part, at the request of other institutions or individuals for the purpose of scholarly research.

I understand that my dissertation may be made electronically available to the public. 
DEVELOPING HUMAN FACTORS METRICS AND TOOLS TO SUPPORT DESIGN AND MANAGEMENT OF PRODUCTION

Doctor of Philosophy, 2016

\author{
Michael Alexander Greig \\ Mechanical and Industrial Engineering \\ Ryerson University
}

\begin{abstract}
This dissertation takes an exploratory look at the role of human factors (HF) metrics within an electronics manufacturing organization by focussing on three objectives: 1) determining company stakeholder views of HF metrics, metrics development and HF application, 2) developing a workstation level HF assessment tool for light assembly work, and 3) creating a tool that reports the level of HF integration and maturity in an organization. Mixed methods were used in an action research framework. Research at the case organization was predominantly qualitative and included field notes, audio recordings, and company documents. Identified gaps between engineering and HF metrics were due to HF metrics focussed more on health and safety measures and activities being completed, gaps in the understanding of HF contributions, and the need for new HF tools to generate reporting measures. Five identified themes affecting HF metrics development included 1) knowledge of engineer processes and of HF principles, 2) connection of metrics to the organization, 3) support of the organization and of the information to the organization, 4) resource availability and limitations, and 5) communication format of metrics information. Collaborative user-centered development of a
\end{abstract}


workstation efficiency evaluator tool helped determine data of interest and effective communication of output variables for users. Design stage inputs create outputs that include HF and system information. The tool performed well in a comparison to an observation-based analysis and also demonstrated tolerance to input errors on workstation outcomes. The developed Human Factors Integration Tool assesses HF maturity across organizational functions. Face and content validity of the tool were tested in field testing and workshops. Participants communicated a need for the tool and its contents. Industry stakeholders found the consensusbased tool helped to establish the status of HF in the organization, plan projects to further develop HF capabilities, and initiate discussions on HF for performance and well-being. The created tools demonstrated approaches to the development of future HF tools. These dissertation findings illustrate the need for more HF metric work, including developing HF measures that contribute to organization metrics, and that the development of HF measures and processes need HF considerations in their development. 


\section{Acknowledgements}

I'd like to thank Dr. Patrick Neumann for providing me the opportunity to expand my boundaries into engineering. Many thanks for your patience as I broke off the writing and research rust. Your guidance has made me better at both tasks. A heartfelt thank you for your understanding and support during the extended and slow progressing finishing stretch. It was greatly appreciated.

Thank you to Drs. Salustri, Searcy, and Zolfaghari for your contributions to not only this dissertation but for your collegiality which has made the department a great place to work and do research. Thank you to Dr. Nancy Black and Dr. Deborah de Lange for providing the external review of the dissertation.

All of this research is not possible without the generous funding from the Natural Sciences and Engineering Research Council of Canada, the Ontario Centres of Excellence and the industry partner. Thank you to the industry research partner for the donation of their generous time as well.

To all of the people that have passed through the lab, thanks for your help, advice, listening ear and breaks from research to enjoy the other parts of life.

A special thanks to Dr. Judy Village for being a great research partner and research role model. The time in the field would have been a great struggle without you.

Many thanks to Dr. Shane Dixon, whether it was research or witty repartee your weekly visit to the lab was a nice change from the norm. Your contributions to helping me understand the qualitative side of research was greatly appreciated.

Thanks to Aileen Lim and Petrit Dode for the travel companionship heading out to the field. It made for a much shorter and more enjoyable ride.

A huge thanks to the administrative staff, Lynn, Shirley, Mimi, Karen, and Lisa. Your smiles, waves and gracious assistance that was delivered with a touch fun made asking for assistance an enjoyable experience. 
To Jastej Gill, thank you for helping me keep the proper perspective and priorities during the challenges of the last year.

For my colleagues at the research partner, you were generous with your time and great to work with. I hope the interaction provided you with as much benefit as it did me.

For my friends who made sure I didn't forget life outside of school - you provided a welcome distraction that I likely all too easily agreed to.

Finally, to my family, you continue to support me, no questions asked and whatever is needed. I can't thank you enough. 


\section{Table of Contents}

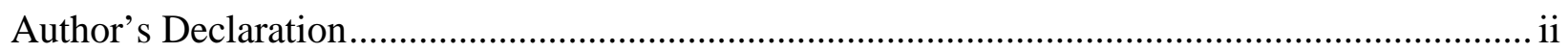

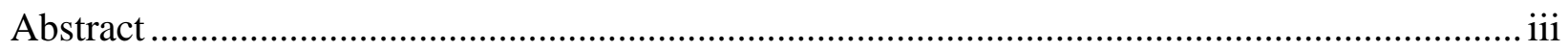

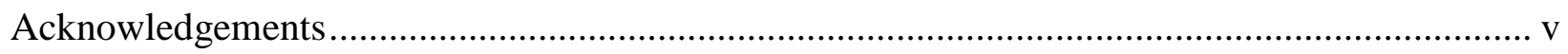

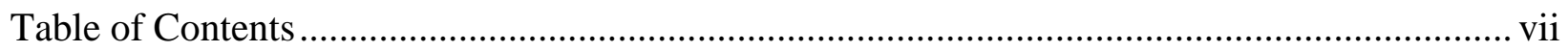

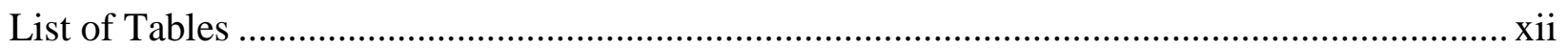

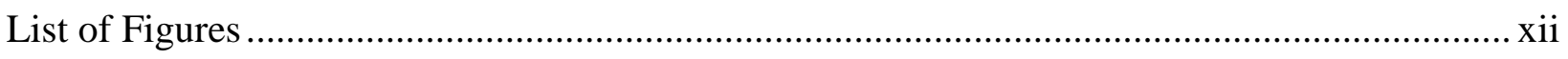

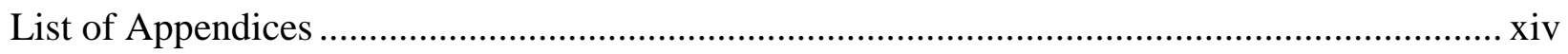

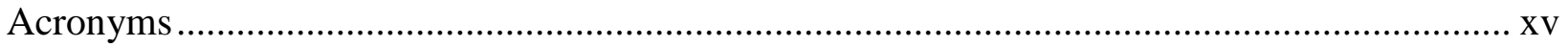

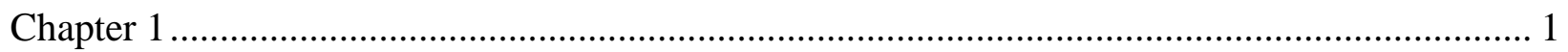

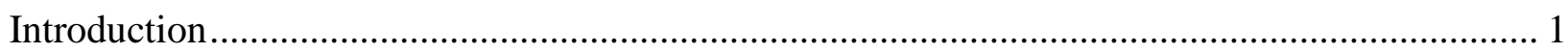

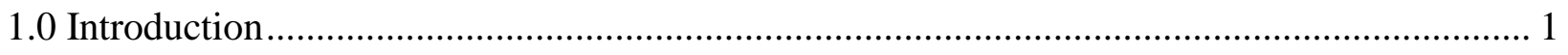

1.1 Human factors is more than injury prevention............................................................... 1

1.2 Human factors needs measurement and reporting ............................................................. 2

1.3 Management through measurement ……………………................................................ 6

1.4 Reporting on human factors .......................................................................................... 10

1.5 Development of workstation level metrics................................................................... 12

1.6 Participative design of HF tools for metrics ................................................................. 15

$1.7 \mathrm{HF}$ from the organization perspective ......................................................................... 17

1.8 Performance management and metrics systems................................................................... 18

1.9 Auditing for HF in an organization .......................................................................... 21

1.10 The human factors metrics fit within performance measurement systems ........................ 22

1.11 Dissertation Aim, Objectives, and Research Questions ...................................................... 23

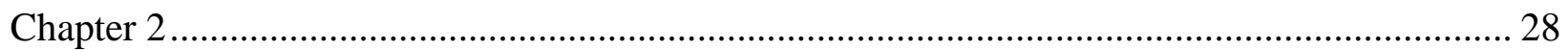

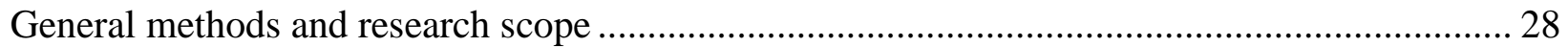

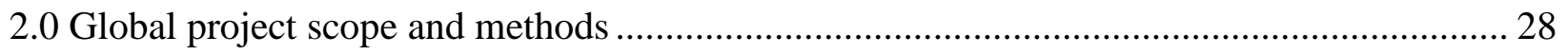

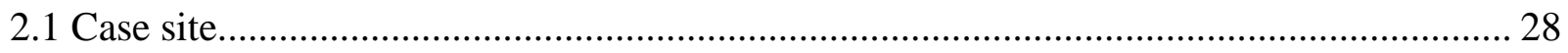

2.2 Study Design ......................................................................................................... 28

2.3 General methods for the case interaction ....................................................................... 29 
Chapter 3

An examination of metrics in a production organization to identify factors affecting current and future human factors metrics 33

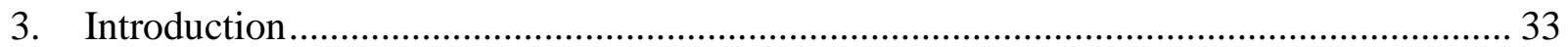

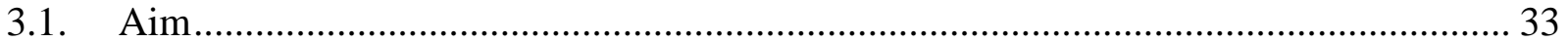

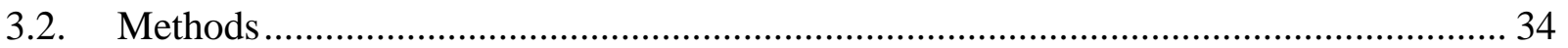

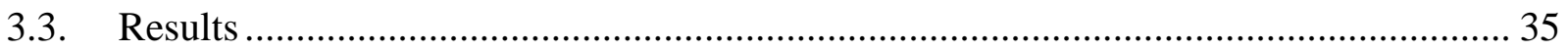

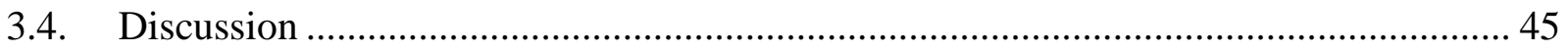

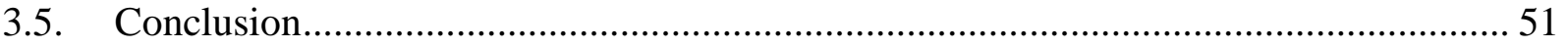

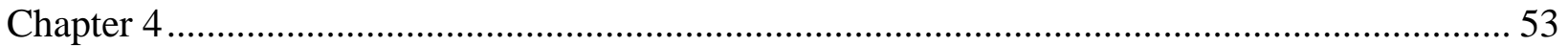

A tool to predict human factors and work element times from workstation layout ................... 53

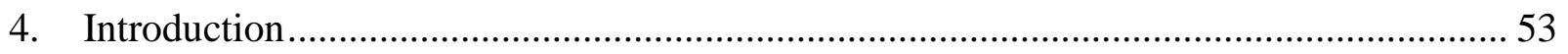

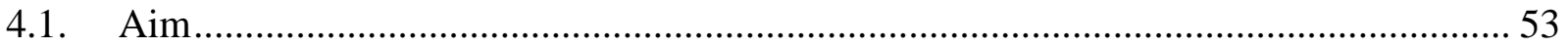

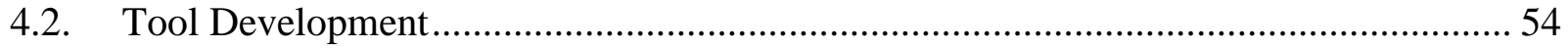

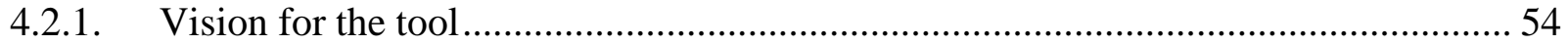

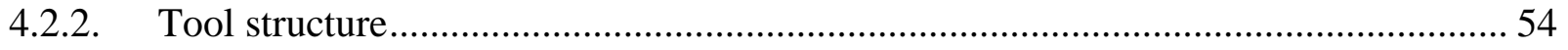

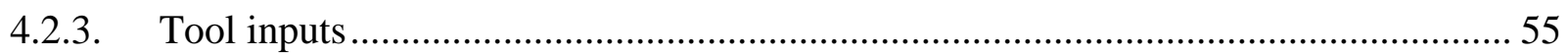

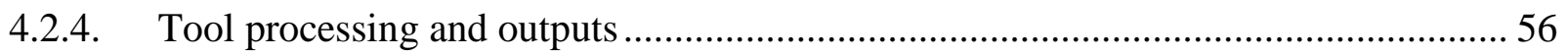

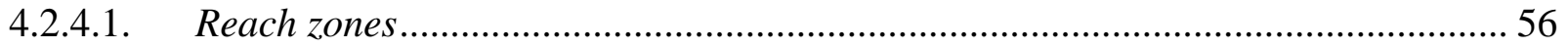

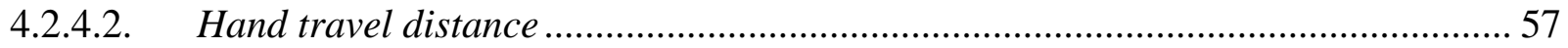

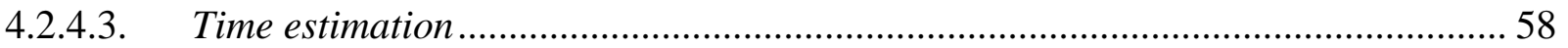

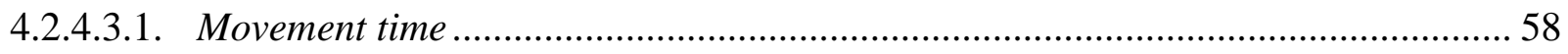

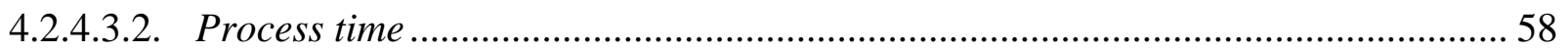

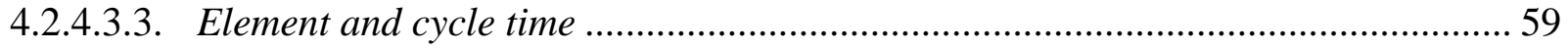

4.2.4.4. $\quad$ Net and cumulative shoulder load ................................................................... 59

4.2.4.4.1. Development of shoulder load models ........................................................... 60

4.2.4.4.2. Translation of tool input values for shoulder model input ................................... 62

4.2.4.4.3. Net and Cumulative Shoulder Load Outputs ................................................... 64

4.3. Case example of application of the tool ............................................................. 64

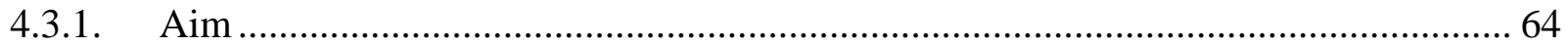




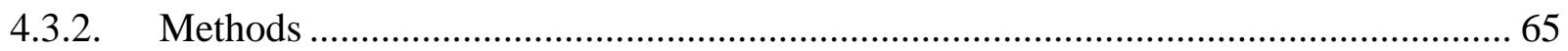

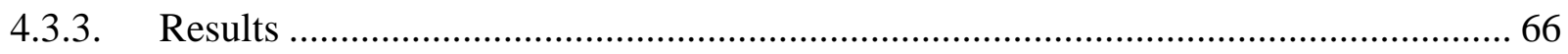

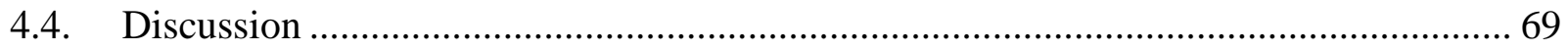

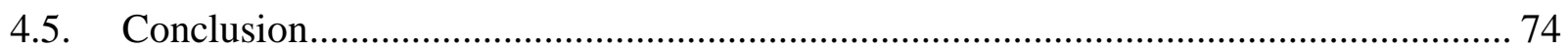

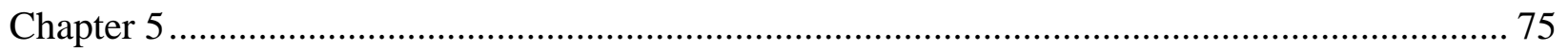

Testing of a workstation efficiency evaluator tool ................................................................... 75

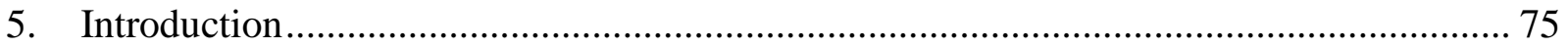

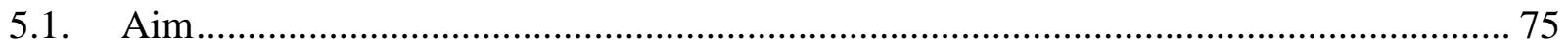

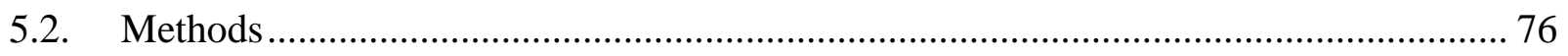

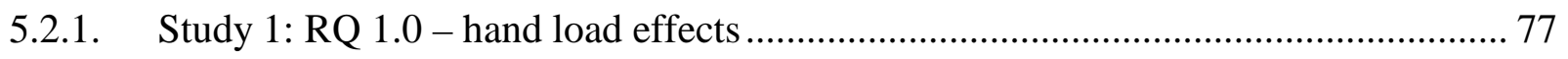

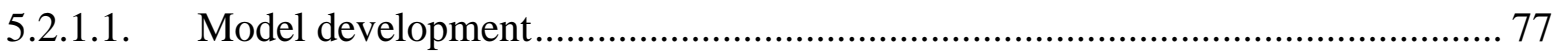

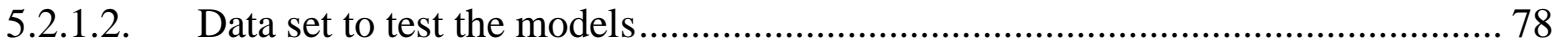

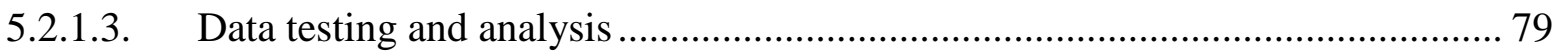

5.2.2. Study 2: RQ 2.0 - hand location measurement error characteristics ........................... 79

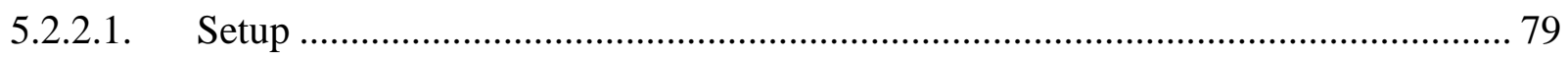

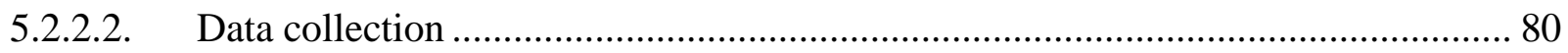

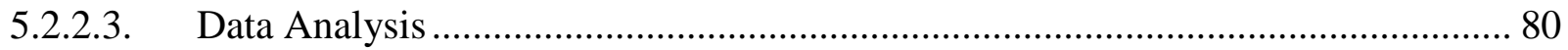

5.2.3. Study 3: RQ 3.0 - Hand location input error effect on load and time outputs............ 81

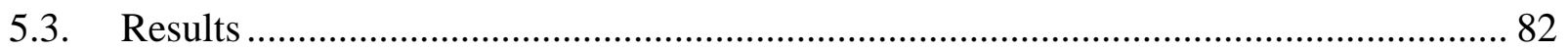

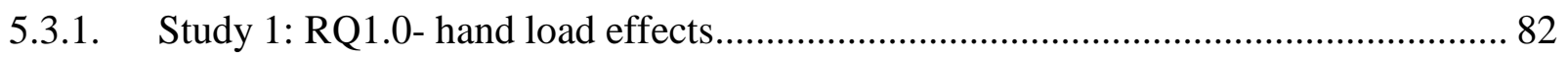

5.3.2. Study 2: RQ 2.0 - hand location measurement error characteristics .......................... 84

5.3.3. Study 3: RQ 3.0 -Hand location input error effect on load and time outputs............ 85

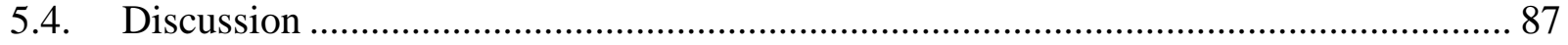

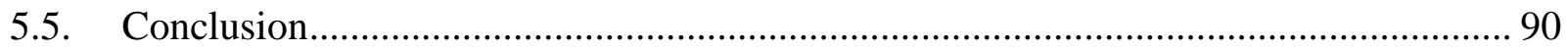

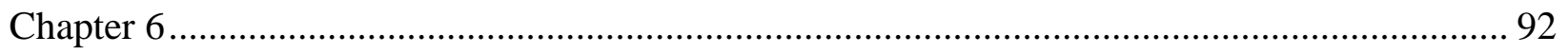

User-centered development of a workstation efficiency evaluator tool ....................................... 92

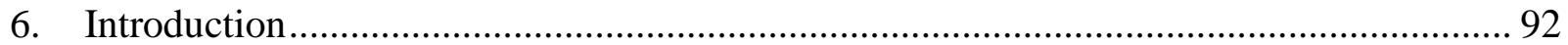

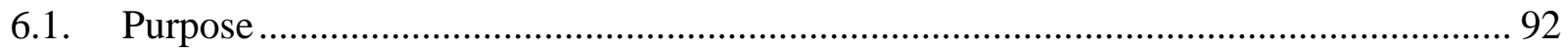

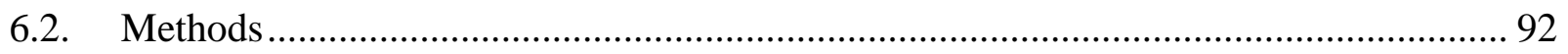

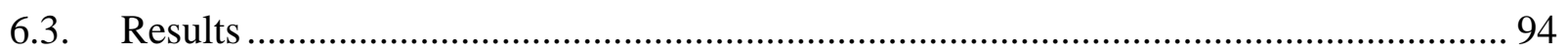




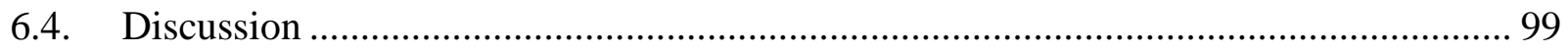

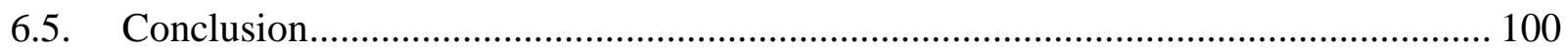

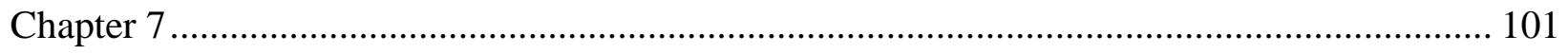

Assessing organizational human factors integration and capability ......................................... 101

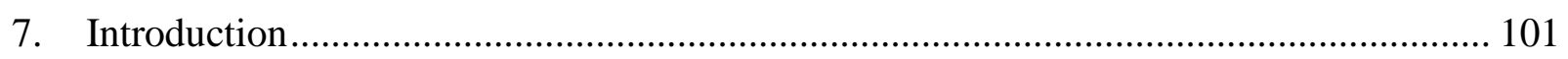

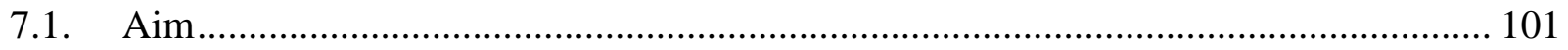

7.2. Methods ................................................................................................................ 101

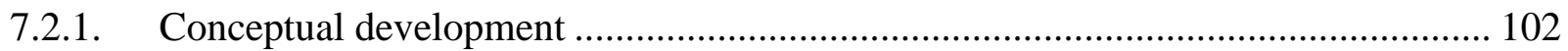

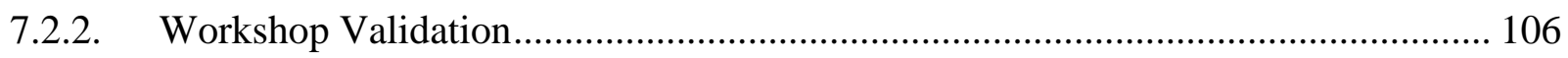

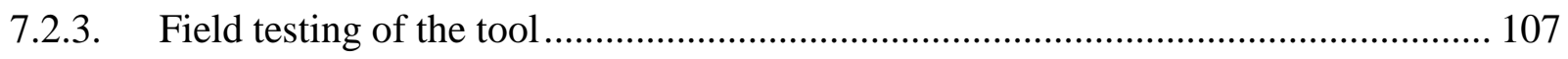

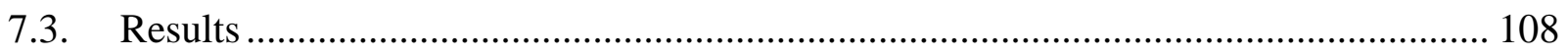

7.3.1. Conceptual development results ............................................................................. 108

7.3.2. Workshop validation results ................................................................................. 110

7.3.3. Field testing results ......................................................................................... 112

7.3.3.1. The need to measure HF across an organization: .................................................. 112

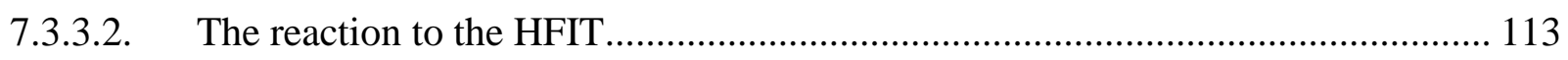

7.3.3.3. Application and benefits of the tool................................................................ 114

7.3.3.4. Tool design issues ........................................................................................ 115

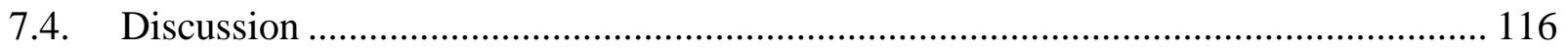

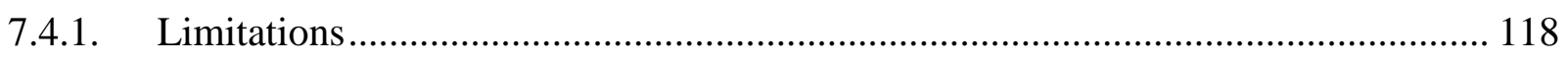

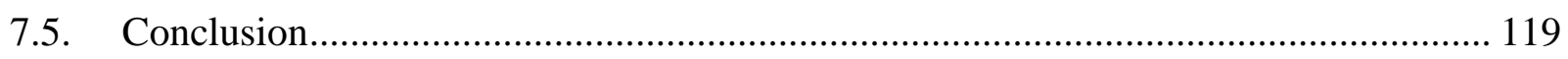

Chapter 8

Discussion, future work, and conclusion ............................................................................ 120

8. Discussion and future work .................................................................................. 120

8.1. Case and methods approach ……………………................................................... 120

8.2. Addressing the three objectives.................................................................................. 121

8.3. General discussion and future work ..................................................................... 127

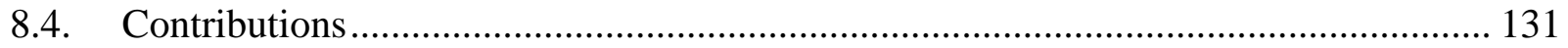

8.5. Conclusion......................................................................................................... 133 


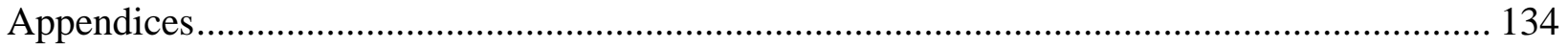

Appendix A. Example of data input tables for WEE tool.......................................................... 134

Appendix B. Coefficients for models with different ranges...................................................... 135

Appendix C. $\mathrm{R}^{2}$ values and equation coefficients for additional models created........................ 137

Appendix D. Example of Training function scoring. ................................................................ 142

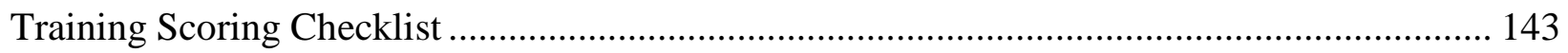

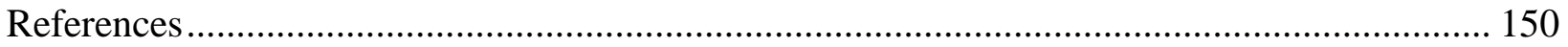




\section{List of Tables}

Table 1. Recommendations for developing HF metrics based on study results. Contributing results are indicated by R\#-p.\#\#, where \# corresponds to the results section and \#\# the page where the result can be found. 50

Table 2 .Equation coefficients and $\mathrm{R}^{2}$ values for the shoulder models used in the tool, where, $\mathrm{X}$ and Y Coefficients are for horizontal and vertical components of the equation, respectively..... 62 Table 3. Translations used to make task hand location values relative to a shoulder joint location.

Table 4. Comparison between assembly task shoulder load, cycle time and cumulative hand travel calculated values for the proposed virtual tool and the observational method used by Neumann et al. (2002). 66

Table 5. Representative average response of the shoulder load outputs to simulated input error for a 5 th percentile female across the 84 point test data set......

Table 6. Predicted time error (ms) and shoulder moment error (Nm) from average and average +/- 1 standard deviation (SD) input measurement error (cm) from Study 2 for all three axes..... 87 Table 7. Example of an assessment rubric for the HF Guidelines HF element of the Training function.

\section{List of Figures}

Figure 1. Sources of human factors metrics in an organization. (Adapted from Neumann and

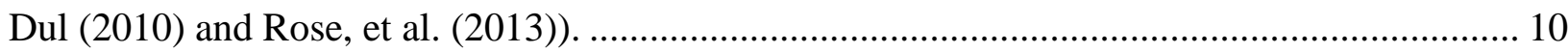

Figure 2. Relationship of dissertation objectives to framework outlined in Figure 1................... 27 Figure 3. The four categories of engineering metrics and indicators that emerged from the review of the case organization. 36

Figure 4. Measures required to describe worker hand location, where: RL is the right-left distance, FB the forward-backward distance, and UD the distance up from an origin at the top, leading edge of the workstation in line with the midline of the worker. Positive values match the direction of arrows. 
Figure 5. Logic flow of the tool. Where \%MVC is the estimated shoulder load as a percentage of the maximum capability for a chosen gender and anthropometry.... 55 Figure 6. Example output summary of a set of work elements for cumulative hand travel and between element hand travel for the right and left hand. Number of element labels reduced to improve clarity. 58

Figure 7. Representative graph showing individual element time contribution to the cumulative work cycle time. Number of element labels reduced to improve clarity. 59

Figure 8. Arm positions used for the three data sets. 61 Figure 9. Workstation set up for the original (left) and new (right) workstations from the system studied by Neumann et al. (2002). 65 Figure 10. Overhead view (of the hand locations, relative to the workstation, required to complete the work elements for the Original Design (A) and New Design (B).

Figure 11. Side view of the hand locations, relative to the workstation, required to complete the work elements for the Original Design (A) and New Design (B). 67

Figure 12. Hand travel between elements and cumulative hand travel for the original workstation (top) and new workstation (bottom). (Note: Element labels changed for simplicity of presentation). 68

Figure 13. Data points within the test data set for the horizontal plane. All points were repeated for $0,10,20,30 \mathrm{~cm}$ above the workstation work surface $(\mathrm{n}=84)$. 78

Figure 14. Examples of a) one of the virtual workstation and b) the matching physical workstation used for data collection. 80 Figure 15. Test measure as a percentage of the reference value for general shoulder load models (GLM) and specific load models (SLM) for a 50th percentile male. 83

Figure 16. Average error $+/$ - one standard deviation for all axes, where Virtual Match, a subset of All Virtual, is the virtual workstation that matches the physical workstation.

Figure 17. Absolute difference in predicted reach time and move time from the reference time value for the four magnitudes of simulated error tested. 86 Figure 18. Significant milestones in tool development.

Figure 19. Framework for the development of the tool, where $n$ functions exist in the organization and $m$ HF elements are contained within a given function. 
Figure 20. List of all Organization Functions and the corresponding HF Elements within each

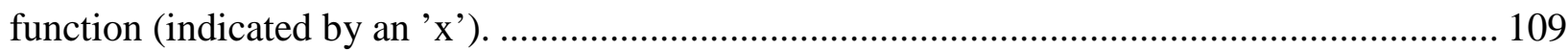
Figure 21. Workshop participant feedback (Average +/- 1 SD; 42 to 45 respondents per item; * denotes significantly different from 4 , a neutral agreement $(\mathrm{p}<0.001))$. Figure 22. Updated framework from Figure 1 to illustrate the number of HF metrics observed in the case organization. Thicker arrows represent more metrics observed. Note that no predictive HF metrics were initially identified.

\section{List of Appendices}

Appendix A. Example of data input tables for WEE tool.................................................. 134

Appendix B. Coefficients for models with different ranges.............................................. 135

Appendix C. $\mathrm{R}^{2}$ values and equation coefficients for additional models created..................... 137

Appendix D. Example of Training function scoring. ......................................................... 142 


\section{Acronyms}

AR - action research

CAD - computer aided design

DHM - digital human modelling

GLM - general load models

HAL-TLV - hand activity level threshold limit value

$\mathrm{HF}$ - human factors

HFIT - human factors integration tool

HFS - human factors specialist

HR - human resources

H\&S - health and safety

IE - industrial engineer

MSD - musculoskeletal disorder

MTM - methods-time measurement

MVC - maximal voluntary contraction

NIOSH - National Institute for Occupational Safety and Health

PMTS - predetermined motion time systems

$\mathrm{RQ}$ - research question

SLM - specific load models

UCD - user-centered design

WEE tool - workstation efficiency evaluator tool 


\section{Chapter 1}

\section{Introduction}

\subsection{Introduction}

\subsection{Human factors is more than injury prevention}

The lifecycle of all products, processes, or systems have some form of human involvement. Human factors (HF) is not always considered in designs even with this integrated human element throughout the lifecycle. The impact of HF is substantial and more than just in injury prevention. HF significantly contributes to worker well-being and performance outcomes such as productivity and quality. Ignoring HF in design and management can thus be costly to the individual, the organization and society. Even with the evidence of the impact of effective consideration of HF in design and management of operations, HF remains underutilized. The management of $\mathrm{HF}$ is one factor among many factors that could be contributing to HF being underutilized. Measurement and reporting metrics of key information is part of effective organization management, with many believing that management is through measurement. Though HF has the potential for many measures, HF is usually poorly managed throughout an organization, especially beyond health and safety or human resources. It is therefore possible that HF measures in their current form are not contributing to existing organization metrics and management systems. Research is therefore needed to understand how HF metrics can be effectively integrated within organization design and management processes. This dissertation reviews the literature on HF metrics, tools, and performance management, and then explores the role of HF metrics in a longitudinal case setting at an electronics development and production organization. 


\subsection{Human factors needs measurement and reporting}

Human factors (HF) has three core characteristics: HF 1) takes a systems approach, 2) is design driven, and 3) focuses on performance and well-being outcomes (Dul et al., 2012). A strategic action proposal by the International Ergonomic Association's Future of Ergonomics Committee has suggested three ways to improve HF awareness - education, strategic partnerships and communication (Dul, et al., 2012). One method for organizations to create organizational awareness is through the use of reporting measures, or metrics, which describe organizational system outcomes and processes. The use of metrics in HF is currently underdeveloped (Neumann et al., 2013). HF metrics are typically specialised, narrowly focussed on health outcomes and worker level health hazards, and are usually disconnected from organizational strategy (Dul and Neumann, 2009). This dissertation explores issues affecting HF metrics as they relate to operations in an electronics production organization. In the context of the case organization the stakeholder views of metrics have been explored and the approaches to the creation of metrics at the workstation level and organization level are investigated.

The contribution of HF, which in this dissertation uses the terms human factors and ergonomics synonymously, is broad based. It has application in the physical, cognitive and organizational domains. The HF discipline aims to understand the "interactions among humans and other elements of the system" with the goal "to optimize well-being and overall performance" (International Ergonomics Association, 2014).

From a management theory perspective, the HF discipline is less Tayloristic in its approach, and more related to the findings and theories of Elton Mayo and the Hawthorne experiment. In Taylorism, the focus in management of manufacturing systems is more on task efficiency, productivity of the average worker, and economic growth (Peaucelle, 2000; Grachev and Rakitsky, 2013). Stereotypically, the Tayloristic scientific management approach overlooks the needs and perspectives of the worker. Conversely, management based on the Hawthorne experiment and work of Mayo is more worker-centric, with concern for the working environment, and interactions between management and employees, to foster performance and well-being simultaneously (Roethlisberger and Dickson, 1966; Wren and Greenwood, 1998). The application of HF is therefore similar to the latter management approach in that the application of HF considers worker performance along with the mental and physical well-being 
of the worker and, as part of maximizing worker performance and well-being, workers are involved throughout the work system development and operation. HF is thus relevant to organizations in a way that is consistent with the sociotechnical system perspective, where the technical and social, or human, aspects of the system are considered as interdependent and are optimized together (Cherns, 1976; Clegg, 2000). Regardless of the management or system approach taken, the exclusion of HF in the design and operation of the work system has the capability to be costly to the worker, organization, and society. HF contributions to well-being and performance need to be effectively communicated.

A lack of application of HF in design can result in worker fatigue, physical discomfort, injury, and a performance reduction, all of which has a cost to the organization. Total system costs associated with lost profits due to poor HF are significant, widespread and not always evident (Rose et al., 2013). Research has shown, for example, that human effects and system effects covary in operations systems (Neumann and Dul, 2010). HF can impact productivity (Yeow and Sen, 2003; Yeow and Sen, 2006), quality (Eklund, 1995; Helander and Burri, 1995; Lin et al., 2001; Yeow and Sen, 2003; Yeow and Sen, 2006; Erdinc and Vayvay, 2008a; Falck et al., 2010; Bosch et al., 2011; Fritzsche et al., 2014) and the ability to implement new technologies (Neumann and Dul, 2010).

Poor consideration of HF when establishing a system can also pose a risk to the health of the worker (Neumann et al., 2002; Neumann et al., 2006) which can lead to musculoskeletal disorders (MSDs) (Bernard, 1997; National Research Council, 2001; Waters et al., 2011; Widanarko et al., 2014). Worker exposure to MSD risk factors are a function of biomechanical, cognitive, psychosocial and cumulative loads. Risk factors are elements that contribute to the emotional, mental, and physical well-being of a worker (Bernard, 1997). Elevated risk factors increase the likelihood for reduced worker well-being and performance. Usually the HF focus in work systems is on physical risk factors that relate primarily to the forces, posture, repetition and time of work being completed (Bernard, 1997; National Research Council, 1999).

The severity of the risk factors depends on the design of the work system. Poor design, such as a stock location that increases hand distance from the body, causes mechanical overload resulting in worker injury (Bernard, 1997; National Research Council, 1999). Similarly, ineffective and cumulatively hazardous or excessively fatiguing worker motions caused by layout can lead to 
rushed work or reduced capability to complete a task, resulting in decreased work quality (Falck, et al., 2010). Healthy worker performance, work quality and system output can also be compromised from poor design HF. One example is workstation layout (Neumann, et al., 2002; Neumann, et al., 2006) where inadequate arrangement of material and positioning of tools can negatively impact work flow and time for task completion, directly resulting in a loss of productivity.

The costs associated with the worker health effects from poor HF in design can be classified as direct or indirect costs (Rose, et al., 2013). Direct costs include those directly associated with the injury outcome, such as sick leave, insurance and medical costs. Indirect costs associated with the replacement of the worker, for example the cost of rehiring, retraining, and loss of experience, are less visible in an organization and challenging to identify because they are embedded within other department costs and expenses (Rose, et al., 2013). Discomfort and fatigue related cost is more difficult to quantify compared to injury, in part because a worker remains on the job but has reduced work capability which can lead to poor system performance. Leading up to an injury the worker can be in a state of pain or discomfort, yet remain present at work but with reduced work output and quality of work. This state of reduced work capability has been termed presenteeism. Meerding et al. (2005) estimated the impact of presenteeism to be as much as a loss of two hours/day in construction workers. However, the cost of presenteeism is typically difficult to determine and without a consensus calculation method (Leigh, 2011). Considering all of the indirect costs together, the cost of poor HF in workstation design can be far more significant than the direct cost associated with injuries alone (Hendrick, 2003; de Looze et al., 2010; Rose, et al., 2013). Combined, direct and indirect costs can reach levels of $1.2 \%$ to $6.2 \%$ of a country’s gross domestic product (Tompa et al., 2008). Total costs have been estimated to be in excess of \$19 billion annually in Canada in 2008 (Gilks and Logan, 2010) and over $\$ 200$ billion in the United States (Leigh, 2011), a value comparable to the 2008 total costs due to cancer (American Cancer Society, 2013). Indirect and direct costs can be mitigated by considering HF in the design stage of development to reduce risk for employees. Incorporating HF considerations into production systems is best done at the design stage where change is easier and more cost effective than retrofitting (Miles and Swift, 1998; Jensen, 2002; Hendrick, 2003; Broberg, 2007; Neumann and Dul, 2010; Edwards and Jensen, 2014). 
Even with the evidence of system benefits and lower investment cost in early application, HF is frequently considered relevant only for injury prevention, disconnected from an organization's strategy considerations and implemented reactively (Jensen, 1997; Neumann and Dul, 2010; Theberge and Neumann, 2013). The lack of HF consideration can also be a result of poor organizational support and the perception that HF is common sense, relevant only to office work, is solely a health and safety issue, and that errors are a function of the person not the design (Perrow, 1983; Helander, 1999; Neumann and Dul, 2010; Theberge and Neumann, 2013). Frequently HF is relegated to the Occupational, Health \& Safety 'sidecar' (Jensen, 1997). As part of the sidecar, HF is not considered to be a key contributor to the core business functions, and deals with simple solutions for minor problems (Jensen, 1997). These perspectives of HF as a non-core business contributor and valuable only for simple solutions leads to the impression that the broad connection of HF to organizations is not well enough understood by stakeholders with varying backgrounds and disciplines.

HF does have a role though in a number of organizational functions because of the HF subcomponents considering the physical, cognitive and psychosocial aspects of the work environment and the dual HF goal of performance and well-being (Dul, et al., 2012). However, this broad based application of HF may make it tough to capture and measure the full impact of $\mathrm{HF}$ and therefore make it a challenge to manage HF in an organization. Kleiner (2004) has suggested that ergonomists are under pressure to "improve global metric improvement”, meaning moving beyond the local improvements of health, quality and productivity of traditional HF metrics to improvements at the organization and society level. HF measurement connection to global metrics could improve HF relevance to non-HF focussed stakeholders. Otherwise, the inability to measure HF and make it more tangible for non-HF specialists can result in HF being misunderstood, glossed over as insignificant, and poorly integrated and managed within an organization (Perrow, 1983; Helander, 1999; de Looze, et al., 2010; Neumann and Dul, 2010). HF outcome measurements, capable of providing plenty of information on health risk and demands imposed at the worker level, do not relate well to organization goals and metrics. A lack of HF measurement capabilities in general, and specifically beyond reporting on employee well-being, reduces the visibility of HF in an organization and makes management of HF a challenge. From an aviation safety perspective, Vogt et al. (2010) have attributed the difficulty 
in measuring HF to be the reason that HF is difficult to manage. Neumann, et al. (2013) contend that there are a number of HF metrics available but that the relevance to system and well-being outcomes and organizational strategy are poorly understood in the design of production systems. This lack of relevance makes HF management challenging. Further, HF could be ignored not only due to a lack of connection of HF to organization goals, but also due to a lack of HF knowledge within the organization or within specific individuals. Though HF may be considered common sense (Helander, 1999), the HF information communicated to other stakeholders has shown a lack of appropriate context in reporting information and in the training content to nonspecialists (Wulff et al., 1999b; Village et al., 2013b; Hall-Andersen and Broberg, 2014; Village et al., 2014b). One method of improving the relation of HF to organizational goals is adapting existing organization tools and practices to include HF information and concepts (e.g. Village, et al., 2014b). Overlooking HF and not effectively considering the human element means that a designed system is not truly optimized. The system may underperform in productivity or quality or it may operate at an unsustainable level that exposes the worker to risks that lead to injury. HF metrics are needed. This dissertation has the objective of improving HF measurement and reporting in the case organization.

\subsection{Management through measurement}

A common management adage is that you can't manage what you can't measure; meaning without information on a process or outcome, such as numbers relative to a target value, you are not able to understand and control what happens. The use of reporting measures can also be referred to as metrics or indicators. Metrics have three functions: control, communication, and improvement (Melnyk et al., 2004) and should include three elements - a defined measure, a sensor that gathers and records data in accordance with the intended measure, and a frequency for measurement and reporting (Bucheim, 2000). Metrics enable the control and evaluation of resource performance; communicate performance inside and outside of an organization; and aid with improvement by identifying gaps between performance and expectation so that appropriate actions can be taken (Melnyk, et al., 2004). The need for organizational performance measurement has been around for centuries (Kaplan, 1984). From the financial accounting for performance that was dominant most of the $20^{\text {th }}$ century, the idea of metrics experienced rapid 
growth through the 1990's and measurement has continued to evolve with technology and perceptions of measurement importance (Kaplan, 1984; Neely et al., 2000).

The concept of metrics can be confusing. Metrics span different groups in an organization and are referred to with different meanings or associated terms (e.g. measure, indicator) (Melnyk, et al., 2004; Melnyk et al., 2005; Huwe, 2010; Keong Choong, 2013). Melnyk, et al. (2004) has identified a metric as a "verifiable measure" that is qualitative or quantitative and considered relative to a reference point. Metrics have both a focus: the resource that is the focus of the metric, for example financial, and a tense: whether the metric reports a performance outcome or predicts future performance. Melnyk, et al. (2004) have also stated that metrics can be thought of as three different, but linked, constructs depending on where they are positioned and clustered in an organization. The constructs include 1) the individual metric, considered the building block, 2) the metrics sets, an aggregate of metrics that direct activities in support of an objective, and 3) the overall performance measurement system which provides coordination, management and continued alignment with the strategic goals of the organization as the metrics evolve. Similarly, Neely et al. (1995) have discussed the idea of measurement with similar terms to the Melnyk, et al. (2004) description of metrics from the perspective of performance management. They considered performance measurement to be the "process of quantifying the efficiency and effectiveness of action" and the metric as the performance measure that is the outcome of the measurement. Further, a performance measurement system was said to be "the set of metrics used to quantify both the efficiency and effectiveness of actions". More attention has been focused on performance management systems and metrics while less attention has been paid to metric sets (Melnyk, et al., 2004). The significance in studying the metrics set is that the set is a cluster of metrics that would be assigned by higher level management to guide or monitor a person's associated work and would also be reflective of a load placed on the person's mental capacity (Melnyk, et al., 2004). From the perspective of HF-related metrics, research is needed in all three metrics constructs to improve HF reporting effectiveness and improve HF integration. This dissertation investigates stakeholder views of metrics to understand issues impacting HF metric integration in the organization.

Developing HF metrics requires knowledge of what is important information to the organization and knowledge about the interests of key stakeholders. Hauser and Katz (1998) identified seven 
steps towards creating good metrics, six of which are related to information gathered from discussions with stakeholders. Understanding stakeholder views and expectations of any current or proposed metrics is needed in order to improve the chance of successful implementation of the metrics. If HF is to be a component within a metrics system of an organization, or its own standalone metrics system, then understanding the views of stakeholders with respect to HF would be important information to know as well. Cognitive mapping is one method that can examine how HF is viewed within an organization and how HF relates to company strategies (Neumann and Village, 2012; Village et al., 2013a). However, the perception of HF and how it relates to company strategies is, by itself, not sufficient enough to develop HF related metrics. It is also important to understand stakeholder views of metrics, in particular HF metrics. Understanding stakeholder views of metrics, and HF metrics specifically, will contribute to establishing a benchmark to the current status of HF understanding in an organization and will also help to provide information to design more relevant HF measures and reporting within that organization. With the need to understand stakeholder views of HF metrics, metrics development and the application of HF within a metrics system in an organization, this dissertation explores stakeholder views of HF metrics in the context of an electronics production organization.

The development of a metric system requires both individual metrics and sets of metrics (Melnyk, et al., 2004) that support the overall metrics system. A range of HF metric possibilities exist that could be used as supporting metrics to a metrics system in an organization. The sources of HF metrics in an organization are shown in Figure 1. This relationship of HF metrics throughout the organization demonstrates that the lagging metrics of one process can be leading metrics for another. In Figure 1 this is shown by design level metrics being leading metrics for the operations. The metrics of operations are outcome to design but are leading metrics for the human and system effects. This means that metrics can have a tense (Melnyk, et al., 2004) and can report information that is both a reflection of the past (outcome) or an estimation of the future (predictive). Reactive, outcome measures are beneficial to understanding how well a system is responding to its current situation, changes that have been completed to the system, or to obtain feedback on how the system was designed. Equally, and potentially more important, are predictive measures. Predictive metrics are beneficial because they are information that can be gathered before a system is running. 
Predictive HF information could be applied at the design stage where it could be used to make adjustments to the system to secure high performance, prevent risk exposure to the worker and prevent costly retrofitting of the system to address these issues (Miles and Swift, 1998; Neumann, 2004). Some virtual HF tools based in heavy industry (e.g. Siemen’s Jack, SantosHuman's Santos) are design stage capable but need specialised training to use, support and interpret the findings (Lockett et al., 2005). Similar virtual tools are less common for light assembly, where there are smaller task loads and fine movement dexterity is necessary to complete assembly tasks. To be most effective, and better manage system-induced risk exposure to the worker, a range of tools that cover all work scenarios need to be able to be used in the design stage of development.

Observation based measures are risk determination measures made from viewing an existing work system. The measures provide leading information for worker risk, but, like injury metrics, they provide important feedback on system outcome performance, in this case the performance outcome of design. Other MSD risk reporting measures include outputs from risk assessment HF tools. These tools are typically observation based and most are difficult to use at the design stage of work system development (David, 2005; Deeney and O'Sullivan, 2009; Takala et al., 2010). Common safety focussed HF metrics report on workers' injury and incident related numbers, which are outcomes of their exposure to a work system.

In the absence of measurement capability in any of the metrics categories in Figure 1, tools are needed to develop new HF metrics. The metrics that could be generated from these tools could help in forming the base measures that support the construction of an informative and beneficial metrics system for an organization. This dissertation has the objective of developing tools for leading HF metrics by creating a workstation level HF assessment tool for light assembly work as well as an organization level assessment tool. 


\section{Human Factors Metrics}

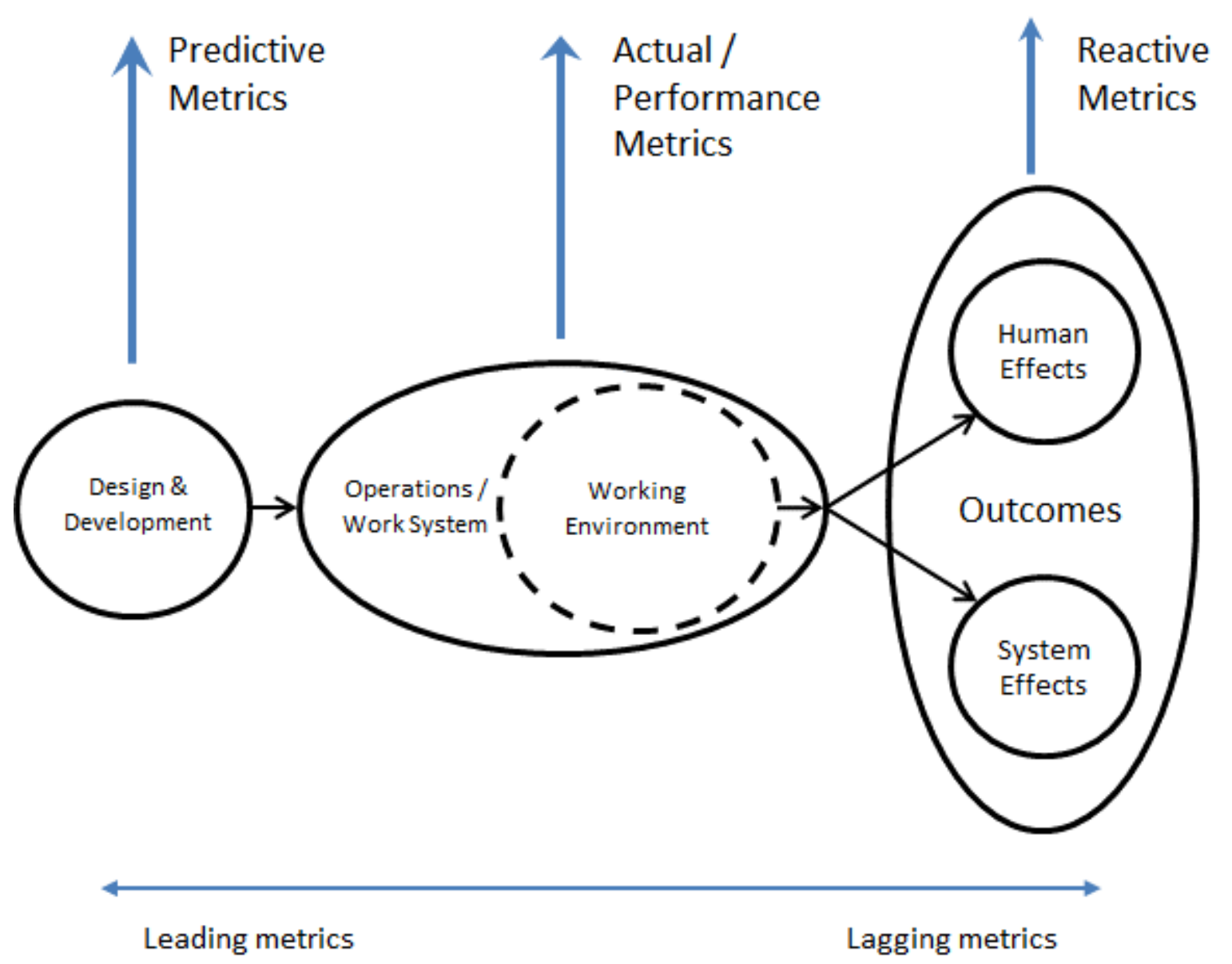

Figure 1. Sources of human factors metrics in an organization. (Adapted from Neumann and Dul (2010) and Rose, et al. (2013)).

\subsection{Reporting on human factors}

HF measures range from broad based, such as perceptions of the working environment (for example, Karasek and Theorell, 1990), to very precise, for example cellular response to load (Allen et al., 2008). Values can be determined from self-reports, observationally, or direct measurement methods (David, 2005). Some measures can report on employee motivation, level of stress and feeling of engagement in the work environment (Larsman and Hanse, 2009). Other measures can provide feedback on body postures relative to acceptable work intensity or frequency levels, hand load level capacity and ability to withstand cumulative load exposure (Takala, et al., 2010). The capability to examine the tissue level response to intermittent or sustained exertions as well as the chemical responses to work evident in the blood stream can 
also be a source of information to understand the effects of work (for example, Garde et al., 2003). A large number of measures can be captured for one worker for any given exertion. There are over 215 pairs of consciously controllable skeletal muscles that could be involved (Wilmore and Costill, 1994). For a single muscle, information could be determined for the acute muscle force, either as an absolute value or relative to a maximum, velocity of movement, tendon forces, amount of recovery required, among other measures. Similarly the joint associated with the muscle could have information collected on its angle, angular velocity, joint moment, ligament forces, and the internal joint forces for shear and compression (Chaffin et al., 2006).

A limit of these biophysical measures, for example ligament and joint forces, is that, stereotypically, the measures are collected by an HF specialist. Further, there is a substantial number of possible HF indicators, which is itself a problem, and for a large number of the measures collected the information is specific to the HF discipline. These measures do not have a context that relates to the work of those outside the HF field and in other organization functions. The lack of relatable context of the HF measures to other stakeholders can mean that the stakeholders do not see a connection to their work which makes an attempt at successful application of the HF specific information beyond the HF field unlikely (Perrow, 1983). As a result, HF can be easily overlooked because the measures are difficult to attach to the strategic concerns of an organization or to design level discussions.

Two development considerations for HF metrics may help improve the connection of HF measures to an organization. One HF metric development consideration is relating the measures to either results (i.e. business outcomes) or the determinants of the results (i.e. factors contributing to the business outcomes) in the organization (Fitzgerald et al., 1991). This has been suggested by Neely, et al. (1995) as a concept to build a performance framework around. Developing HF metrics to fit the results and determinants could therefore improve the chance of HF metrics integration. The second HF metric development consideration is meeting the measurement priorities of the managers. Managers are often looking for quick measures that are 'good enough' and provide quick information in their specific context (Melnyk, et al., 2004). Simon (1956) has termed this accepting of the satisfactory instead of the optimal as 'satisficing'. That managers have less time for metrics development and less desire for generalizability is in 
contrast to researchers, who often spend time to ensure valid and generalizable results (Melnyk, et al., 2004). . Researchers developing HF tools for metrics thus need to keep in mind that users may not need the rigour and accuracy necessary for good research. For example, tools to determine precise joint loads are less important than a threshold ‘Go' or 'No go' outcome, especially if a lot of effort is required to achieve the result. HF practitioners in the field and researchers alike need to remember that measures they create can benefit from addressing these two considerations in the creation of metrics.

HF measures shared beyond HF specialists need to be relevant to the organization. The measures need to provide commentary on the system induced risks that affect the worker wellbeing and simultaneously demonstrate how that risk impacts the organizational system. Even though the metric may not be a direct contributor to organizational outcomes, the indirect contribution of HF as a determinant is beneficial provided the information is in the proper context for others to understand. Improving the relevance of HF in an organization can also potentially be enhanced by improving stakeholder support through active discussions with key stakeholders receiving and reviewing the metrics information. Understanding the important focus of a senior manager, or upper level organization strategies, enables tailoring of the metrics to support their agendas. Information can then be provided that matches the needs of the different levels of management and decision stages (Fitzgerald, et al., 1991). As highlighted by Fitzgerald, et al. (1991), information requirements vary because "there is a difference between information for diagnosis and information for control and performance measurement”.

The improved understanding of HF measures will help to move HF beyond the status quo and achieve the goal of unlocking HF potential and securing high levels of system performance without injury, as suggested by the HF research community (Neumann and Dul, 2010; Dul, et al., 2012). The development of HF metrics, and understanding the methods that the metrics are created, needs further research in order to improve HF metric relevance to organizations. This dissertation explores stakeholder views of metrics and co-develops HF tools to better understand how HF metrics can be related better to the organization and its stakeholders.

\subsection{Development of workstation level metrics}

Designing effective light assembly workstations is challenging but crucial for maximising assembly system performance. Balancing task time and worker load is critical for a system to 
perform at its maximal capacity. Typical light assembly is low load, high repetition, fast paced work that is commonly found in electronics manufacturing (Belbin, 1955; Heilala et al., 2008). Light assembly tasks are usually confined to a relatively small workstation area, meaning all tasks and stock are arranged to be within a short reach. With limited workstation space, engineers have to consider all layout options in order to maximize performance and minimize risk to the system and worker. Often the HF component of a workstation is not considered as well or as early in the design process as it should be (Perrow, 1983; Neumann, et al., 2002; Helander, 2006; Broberg, 2007). As a result, both the human and system performance outcomes of the workstation are compromised (Helander and Burri, 1995; Neumann and Dul, 2010). This research develops and tests a design-stage tool to assess light assembly workstation layouts for the impact on system performance and worker well-being.

Individual metrics provide the foundation of information of the metrics systems. Kleiner (2004) has identified traditional HF metrics as productivity, health and safety, quality of work life, performance time, and errors. Common safety oriented lagging HF metrics include injury, incident rate and associated lost or restricted work time. HF metrics are also possible from the outputs of risk analysis tools.

In the absence of an appropriate indicator for decision makers, or a method of assessment, a measurement approach needs to be created for HF practitioners and relevant stakeholders. Ideally this is a tool or method that can be used to predict risk exposure to the worker and the system during design. Contributing in the design stage allows for early changes to avoid the expensive cost and inertia to change common with retrofitting (Miles and Swift, 1998; Neumann and Dul, 2010). A number of HF tools are available to predict risk. They range in complexity, cost and application.

Most HF tools are observation based, meaning they are a reactive measure to design work, and proactive to injury (Takala, et al., 2010). For example, a worker and existing workstation are needed to properly use observational tools like RULA (McAtamney and Corlett, 1993), REBA (Hignett and McAtamney, 2000), and the ACGIH HAL (American Congress of Governmental Industrial Hygienists, 2001; Marras and Karwowski, 2006). Observational HF tools are affordable and easy to use but require a mock-up or existing system in place in order to complete an assessment. These types of tools provide appropriate feedback after a system has been 
designed but do not support design level decisions. Nor do they comment on system performance.

The virtual human factors (VHF) subset of HF tools, as highlighted by Perez and Neumann (2015), are capable of design level reviews. VHF tools include predetermined motion time systems (PMTS), discrete event simulation (DES), digital human models (DHMs), and virtual reality (VR) (Perez and Neumann, 2015). VR tools require expensive equipment and digital or physical mock-ups of the work environment to interact with. DHMs, which range in complexity, environmental dimensions (2D versus $3 \mathrm{D}$ ), cost and use of CAD environment drawings, are capable of providing more comprehensive albeit more complex specialist review (e.g. Jack, RAMSIS) (Perez and Neumann, 2015). The use of VHF tools is limited in industry due to their operating costs and the need for skilled individuals to operate the tool and interpret the results (Lockett, et al., 2005). Virtual, or design level HF tools are therefore needed that are, among other criteria, cost effective, easy to use and improve the connection between HF specialists and engineers (Wells et al., 2007; Village, et al., 2014b; Perez and Neumann, 2015).The development of a cost effective virtual method is needed for HF in general and especially for light assembly. This dissertation develops a simple and inexpensive virtual workstation level tool that predicts light assembly task demands on the worker in the layout design stage.

The development of HF tools has improved the assessment capabilities of HF practitioners yet HF tools have not been widely adopted by non-HF specialists. Effective tool development needs to be directed by design criteria and co-created with the end user to ensure the tool is relevant and applicable to HF practitioners and engineers. Perez and Neumann (2015) and Village, et al. (2014b) have both highlighted tool requirements for VHF and modified industrial engineering tools with HF content, respectively. Three common themes between the two studies were ease of use of the tool, its usefulness to organizational processes and its ability for reports/results presentation and interpretation. In their case study at an electronics manufacturer, Village, et al. (2014b) also highlighted four phases that improve tool adaptation and integration into engineering design, namely understanding the design process, understanding the important metrics driving business performance, understanding current engineering tools to meet business goals, and fitting tools that provide important metrics for business performance to the design process. A recurring theme was developing an HF tool with engineering relevance. 
Few of the existing HF tools have incorporated engineering measures as part of the tool inputs or outputs. Likewise few engineering tools have incorporated HF. Time aspects have been identified as a key connection between ergonomists and engineers in order to understand system and worker related performance and risk (Wells, et al., 2007). Combining time with an HF analysis can provide system related information (e.g. cycle time) and allow scaling of discrete HF information to cumulative exposures. Few HF tools have been designed to capitalize on this important connection. Some researchers have started to integrate DHMs and predictive models with discrete event simulation (DES) and predetermined motion time systems (PMTS) in order to improve the time component and create a more robust analysis of work capabilities (Laring et al., 2005; Kazmierczak et al., 2007; Dode, 2012; Perez and Neumann, 2015). ErgoSAM is one tool that has been developed as a cost effective complement to Methods-Time Measurement (MTM) analysis to predict ergonomic quality at the design stage (Laring, et al., 2005). Similarly, HF and time elements have been used to design workstations and balance work processes. For example, Ben-Gal and Bukchin (2002) combined MTM with worker posture, energy expenditure and lifting criteria to virtually design a fruit packing workstation, while Carnahan et al. (2001) used cycle time information and grip strength requirements in line balancing heuristics. Braun et al. (1996) addressed the design of a light assembly workstation by applying MTM-1 along with worker anthropometrics to determine economic and ergonomic aspects. The previous examples aside, tools and research that help with the prediction of the effects of HF on operator and system outcomes in work station design are generally sparse. More work is required to develop

predictive HF tools, in particular for light assembly layout, in order to improve HF understanding and maximise system output. A cost effective tool integrating the tool design requirements and ideas proposed by Wells, et al. (2007), Perez and Neumann (2015), and Village, et al. (2014b) is needed for assessing light assembly workstation design. This dissertation develops a tool to predict both the demands on the worker and the time demands on the system in the workstation layout design stage of light assembly.

\subsection{Participative design of HF tools for metrics}

HF tool designs that do not completely consider the needs of non-HF specialized users (e.g. workplace designers) could lead to a lack of HF tool adoption. This tool design disconnect could be because HF researchers and practitioners create tools that focus solely on MSD risk without 
providing proper usability and context of the results for users less familiar with HF principles. Co-development of tools with the future users should further improve development and verify the tool requirement claims made by Wells, et al. (2007), Perez and Neumann (2015), and Village, et al. (2014b). Understanding the context of tool use, crucial to good design, is improved through direct contact with users (Kujala, 2003). Jointly designing tools, or at the least getting user feedback at key points in the design stage could assist with tool adoption.

User-centered design (UCD) is one method to engage users directly in development. UCD provides a cyclic, iterative design interaction of prototyping and usability testing between a developer and product end user (Kujala, 2003). The aim of involving potential HF tool users through UCD is to develop useful and usable products. Ergonomic design initiatives following UCD protocols can produce outcomes that effectively align processes and products with users to great positive effect, improving worker health and organizational performance (Toccafondi et al., 2012). Conversely, UCD can be challenging. Conflicting interests can impede the design process and its participatory nature (Garrety and Badham, 1999). In some cases stakeholders need to employ creative communication and development tactics to facilitate the alignment of interests (Waterson and Kolose, 2010). The UCD literature heightens our awareness of both the facilitators and barriers that shape the UCD process. It also shows that one needs to be aware of both the context in which the UCD process is embedded and how the context can shape both the outcomes of design efforts and the process itself. HF practitioners promote the involvement of workers in system design. User-centered design in HF tool development should not be considered differently.

The cyclic nature of UCD tool development, where feedback and review directs the next iteration of development, is similar to the cyclic nature of the action research (AR) process. Like the developer-user(s) interaction, AR uses a similar researcher-field participant(s) pairing to develop an understanding of an issue of common interest. Neumann et al. (2012) believe that AR can be a valuable method, working alongside an organisation to study and achieve ergonomics application. The AR similarities with UCD mean that the two methods can be used in concert to both develop and understand the development of HF tools in the field. Future tools can potentially be developed more rapidly through the increase in understanding of key themes and indicators facilitating successful tool creation. This dissertation applies a UCD approach to 
HF tool development of a workstation assessment tool and an organizational assessment tool. The tool development includes the design criteria and content that have been suggested by users engaged in the tool development process, and a simultaneous examination of the stakeholder feedback to understand development issues for future tool creators.

\subsection{HF from the organization perspective}

Consultants reviewing organizations for HF capability use approaches that are typically focused on injury prevention within the health and safety domain. This is a logical choice based on Canadian ergonomists and HF practitioners working predominantly in health and safety or human resources (Theberge and Neumann, 2013). Less common are assessments of HF integration in other organization domains, for example the production system (Bierwirth et al., 2010). The common focus of HF on well-being reinforces HF as only a health benefit resulting in HF contributions being underexploited because it demonstrates a lack of connection to other organization interests (Helander, 1999; Hägg, 2003; Neumann and Dul, 2010; Dul, et al., 2012; Theberge and Neumann, 2013). HF has been identified as being more than a health benefit by taking a systems approach, being design driven, and focusing on both performance and wellbeing (Dul, et al., 2012). A sub-discipline of HF, macroergonomics, considers HF from the system perspective.

Macroergonomics, the study and design of work systems with a focus on the interaction of the organization and system, can assist with organizational change because of its systems approach to HF (Kleiner, 2006; Wilson, 2014). It considers macro issues of the organization and environment along with the ergonomic micro issues (Kleiner, 2004) and shares similarities with broad, holistic approaches like quality management and general management (Zink and Seibert, 2009). The sociotechnical framework of macroergonomics means that performance is multidimensional with many criteria and measurements throughout the work system (Kleiner, 1996; Kleiner, 2004). This framework focuses on the optimization of the work system by considering the interactions between social, technical, and environmental variables (Kleiner, 2004). Consideration of HF from a macro perspective can thus impact organization culture and result in substantial performance improvement (Hendrick and Kleiner (2001) as cited in Kleiner (2004)). Even with this demonstrated system connection few performance measurement systems include reporting of HF information, or of information about HF contributions beyond 
employee engagement and injury tracking. Part of the reason for not reporting on HF at the organization level is that very little HF information appears to be available at the organization level. Methods for assessing and reporting the status and impact of HF throughout an organization are needed if HF is to become an effectively managed contributor to organizational level goals (Neumann and Village, 2012; Neumann, et al., 2013). A method of assessing and documenting the integration and maturity of HF in the whole organization is not currently available. An approach that captures the HF contributions to the whole organization is needed to address this shortcoming. This dissertation addresses this issue with the development of an organization assessment tool focusing on HF integration and maturity.

\subsection{Performance management and metrics systems}

Performance management systems develop and evolve with changes in the business mindset. A lack of significant management accounting developments from 1925 to the 1980's resulted in traditional accounting measures being increasingly criticised as being inadequate for business management (Johnson and Kaplan (1987) as cited in Bourne et al. (2003)). Productivity, in the form of labour, machinery and resource productivity, was of significant concern during this period and financial and operation metrics accounted for more than ninety percent of metrics on any performance management system scorecard (Brown, 2007). These scorecard measures, which typically provide a summary of an organization's performance to upper level managers, had become too narrowly focused in the organization and were not effective (Bourne, et al., 2003). The ineffectiveness of traditional accounting performance measures was summarised by Bourne, et al. (2003) to be due to the measures 1) not matching more modern manufacturing techniques (Kaplan, 1986; Turney and Anderson, 1989), 2) leading organizations into short-term decision making (Kaplan, 1984; Kaplan, 1986) and 3) not considering the global performance of the organization.

The addition of quality related metrics in the 1980's, influenced in part by the simultaneous focus on Japanese quality systems and total quality management (see, for example, Hackman and Wageman, 1995; Liker, 2004), was a second phase in the evolution of metrics systems and the group of metrics on the organization scorecard (Brown, 2007). Scorecard focus shifted to improving efficiency and quality of products and processes along with increasing customer satisfaction. Improved information gathering capabilities allowed larger metrics summary 
scorecards to be created, which was believed to be symbolic of an intelligently run company (Brown, 2007). This style of performance management proved insufficient and led to the balanced scorecards of the mid 1990's (Bourne, et al., 2003). The proposed balanced metrics captured financial and nonfinancial interests with information from customers, shareholders and employees (Brown, 2007). Fewer metrics, 15-20, were being used per scorecard compared to earlier metric systems because the volume of information was approaching the threshold an individual could handle (Brown, 2007). Examples of balanced measurement systems frameworks, highlighted by Bourne, et al. (2003), include the supportive performance measures matrix (Keegan et al., 1989), the Balanced Scorecard (Kaplan and Norton, 1992), the SMART pyramid (Cross and Lynch, 1988), the Results/Determinants Matrix (Fitzgerald, et al. (1991); Fitzgerald and Moon (1996) as cited in Bourne, et al. (2003)) , and the Performance Prism (Neely et al. (2002) as cited in Bourne, et al. (2003)).

Although balanced measurement systems look broadly inside and outside of an organization, HF within the organization is rarely mentioned. In some measurement systems there is no direct mention of HF, or even internal stakeholders, though points for HF integration exist (for example Kaplan and Norton, 1992; Flapper et al., 1996; Bititci et al., 1997; Bititci et al., 2000). Other measurement systems have a minor connection to HF because employee loyalty and satisfaction are considered due to the connection to customers (for example Brown, 1996; Epstein and Westbrook, 2001). While in some measurement systems HF consideration is a little more substantial and is addressed through human resources, or includes the notions of training, participative approaches, clear objectives, incentives, or values and culture (for example Fitzgerald, et al., 1991; Bititci, et al., 1997; Epstein and Westbrook, 2001; Andersson et al., 2006). The triple bottom line, performance measurement considering environment and social objectives along with economic considerations (Elkington, 1998), and corporate social responsibility performance reporting approaches have perhaps the greatest potential for HF reporting. However, a review by Searcy et al. (2016) for work environment indicators in corporate social responsibility reporting demonstrated a need for a broader scope of work environment variables, especially those that are not regulated, while the triple bottom line has been said to contain 'vague' and 'meaningless principles' (Norman and MacDonald, 2004) which leaves the HF related reporting requirements up to individual interpretation. Brown (1996) has commented that well-being and satisfaction should be on all scorecards, but that well- 
being and satisfaction was the far weakest component and that few had figured out how it could be included. The HF content in measurement systems is noticeably minor and varied in focus. Where HF content exists it deals with employee development and human resources with no mention of the application of HF in design. An approach to considering HF contribution to an organization as a whole is needed. This dissertation has an objective of creating a tool that reports the level of HF integration maturity in an organization to improve the understanding of HF across an organization.

The development of 'balanced scorecard metrics system frameworks' demonstrated the need to consider a broad base of information beyond financial outcomes that contribute to organizational success. Accordingly, a broad range of metrics that assess core and ancillary business functions were needed in these systems. The suggested focus of these metrics rarely refer to HF. A general measure focus is suggested in some instances. For example Fitzgerald and Moon (1996) (as cited in Neely, et al. (1995)) classified two types of important measures that reflect contributors to the business (determinants) and outcomes of the business (results) for their performance system. While others are more specific. For example, in the Balanced Scorecard there are four key organization perspectives to monitor: financial, customer, internal business, and innovation and learning (Kaplan and Norton, 1992). In the general measure focus of the determinants and results, HF would have to be understood to contribute to the business beyond health and safety. In the specific measures of the Balanced Scorecard HF would need to be seen to contribute to those specific perspectives. Hudson et al. (2001) have compiled six critical dimensions of performance for creating measures that cover all aspects of business. The dimensions included quality, time, flexibility, finance, customer satisfaction and human resources. HF principles were most closely matched to the terms in the human resources dimension which included: employee relationships, employee involvement, workforce, employee skills, learning, labour efficiency, quality of work life, resource utilisation, productivity. With no connection of HF to the terms in the other dimensions it is understandable how the role of $\mathrm{HF}$ in an organization beyond human resources can be easily overlooked. Without the experience of an HF practitioner to identify points of HF influence, or the specific identification of HF as a term in any of these dimensions, it is unlikely that HF consideration as an outcome, contributor to an outcome or continuous improvement process will be considered. Not only does this indicate the need for more HF metrics but this also indicates the need for an approach to relate HF to the 
whole organization. This dissertation develops an approach to assessing the HF integration maturity within the case organization.

\subsection{Auditing for HF in an organization}

Performance measurement systems measure strategy implementation success and use collected information to challenge strategic assumptions (Bourne et al., 2000), as well as align and coordinate metrics (Melnyk, et al., 2005). In part, performance measurement systems help to provide organizational direction and create change as required. Audits of organization sub units,

for example ISO audits for quality and environmental standards, can guide future processes and ensure standards are met. Organization level audits provide the ability to benchmark performance, track and plan continuous improvement initiatives, and measure compliance with system standards and requirements. Audits have been used to standardise procedures and requirements across businesses and industries. The International Organization for Standardization (ISO) has created a number of standards to bring consistency to organizational processes and provide assurance to the customers of quality of work. Audits have been created based on these standards to audit performance management system elements and compliance for example, see ISO 19011 (International Organization for Standardization, 2002) and the Occupational Health and Safety Management Audit review by Bigelow and Robson (2005). A method to score the integration of HF in an organization is not readily available. Nor is there a standard model for HF integration. This dissertation develops a tool to assess HF integration and maturity within an organization based on subject matter expert validated content.

Similar to standards-based audits, quality performance models assess companies for compliance to constructs of elite business performance with a focus on the customer. For example, the Malcolm Baldridge National Quality Award (MBNQA) has in the past scored a company over 1000 possible points, 300 of which related to the customer (Garvin, 1991; Neely, et al., 1995). The European Foundation for Quality Management (EFQM) Excellence Model (European Foundation for Quality Management, 2013) has been constructed with the belief that successful organizations need a good management structure that can be guided by self-assessment of nine determinants that strengthen partnerships, resources and processes (Gómez et al., 2011). The HF component in the ISO system auditing and the Baldridge and EFQM systems are minor. Human Resource Utilization accounts for 15\% of the total 1991 MBNQA score with "human 
resource management” and “employee well-being and morale” accounting for 2\% and 2.5\% of the total (Garvin, 1991). These components are all reactive HF outcomes meaning that HF would not contribute to design within an organization. Lee (2005) has highlighted the MBNQA criteria as "requiring ergonomic approaches" for both quality and product design. A search for the word "ergonomic" or "human factor” in a 2011-2012 MBNQA Criteria for Performance Excellence document (National Institute of Standards and Technology, 2011) did not return a specific match to the terms. Inspection of the information showed that the reference to HF is related to safety and comments on workforce performance. The indirect reference to HF would likely need a subject matter expert to create the connection between productivity and HF, meaning HF would still be perceived as primarily a well-being benefit. With the cost implications of poor HF spanning a large part of an organization (Rose, et al., 2013), the ability to understand how well HF is performing throughout an organization is needed. ISO-based or performance excellence-based auditing in their current form would not appear to be the best solution for assessing HF application in an organization. This dissertation creates an approach to assess HF integration in an organization.

\subsection{The human factors metrics fit within performance measurement systems}

Given how broad metrics systems have become, where might HF fit in? Keegan, et al. (1989) suggest connecting measures to corporate strategy but add that the measure does not have to directly reflect cost but can be a cost driver. Safety is often presented as a key organization concern and can have significant cost implications. The safety and injury prevention focus has been driven nationally and provincially, for example by the Workplace Safety and Insurance Board of Ontario or Ministry of Labour, to take care of the worker. Failing to properly protect the worker can result in significant financial penalties. The measure that typically provides organizations with their safety performance from the worker perspective is incident rates. Other HF measures that fit well within the safety focus in an organization are injury counts and lost or restricted work days. These HF-related measures reflect a reactive, lagging connection of HF to organizational key performance indicators. Missing from within safety is HF-related performance measures relating worker capabilities to system output. The system impact of HF has been shown to be far broader than injuries alone (Goggins et al., 2008; Neumann and Dul, 2010; Rose, et al., 2013). The difference from workstation level metrics to organization level 
metrics is highlighted by the Kleiner (2004) statement that the "nature of success measures changes” in the movement from micro- to macroergonomics. Development of HF metrics is needed that allows the estimation and reporting of HF performance across organizational processes. Figure 1 illustrates the connection of HF metrics throughout an organization and demonstrates that HF metrics could contribute to different parts of the organization. This dissertation develops HF metrics at the workstation and organizational level. The user-centred design development of these tools helped to establish metrics that were relevant to the different needs of stakeholders in the organization.

The need for measurement capabilities at a workstation level is matched by a similar need to measure the integration of HF into organization processes, and the maturity of HF integration in the whole organization. Knowing the integration and maturity of HF in an organization enables an understanding of the potential capability for different functions and employees in the organization to absorb HF information and work with HF measures and processes. The knowledge can help guide an organization to continuously improve their HF capability, increase the level of HF integration, and provide feedback on change that is happening. Macroergonomics is an approach that considers HF at an organizational level but assessment approaches are not common. The style of assessment at the organization level could be conceptually similar to standards audit systems (e.g. ISO 9000 or ISO 14000; International Organization for Standardization (2013)) and performance management systems (e.g. Malcolm Baldridge Performance Excellence Model (National Institute of Standards and Technology, 2012), EFQM Excellence Model (European Foundation for Quality Management, 2013)) which verify that key steps and processes are in place. However, audit systems do not deal with HF directly. Should an assessment system be created to examine HF from the perspective of the organization, the outcomes from an assessment could be used to enhance the ability to manage HF in the organization. This dissertation will addresses the need to create a means to determine the level of HF integration and maturity at the organization level by laying out the groundwork of an assessment approach.

\subsection{Dissertation Aim, Objectives, and Research Questions}

This dissertation aims to contribute to understanding how HF can be integrated into the design of production systems in a case electronics development and production organization. The focus is 
specifically on the role of HF metrics within a production system design and production organization. To achieve this aim, with this focus, the research will investigate the factors impacting the views of HF metrics and HF metric development, and how HF tools at the workstation and organization level can contribute to HF metrics and the dual HF goals of system performance and employee well-being. Combining performance and well-being goals in tool development aims to improve the relevance of HF to multiple organization outcomes. This strengthens the position of HF as a means to an end instead of an end in and of itself. In this case HF is not seen as a priority to worker well-being or separately system performance but as a general contributor to organization success as a whole. This research was completed for the following objectives by addressing the specific research questions (RQs).

\section{Objective 1: Determine the company stakeholder views of HF metrics, metrics development and $\mathrm{HF}$ application}

Investigating the integration of HF into engineering processes with the organization necessitated the following question:

Research Question 1.1 - How do HF metrics compare to engineering metrics in the organization?

To better understand how metrics are viewed in the case organization the question was asked:

Research Question 1.2 - How do stakeholders view metrics in the organization?

In the attempt to improve the future integration of HF and engineering metrics it is important to understand the factors that impact the relationship established in RQ 1.1 by asking the following:

Research Question 1.3 - What factors affect how HF metrics relate to engineering stakeholders in the organization?

With the knowledge gained from the previous three RQs more relevant HF metrics can be developed. It is necessary to understand issues affecting metrics in order to better understand how HF metrics should be created. This leads to the following question:

Research Question 1.4 - What issues might affect efforts to develop and integrate HF metrics in the organization?

\section{Objective 2: Develop a workstation level HF assessment tool for light assembly work} In the absence of a simple, virtual HF assessment tool for light assembly, it was necessary to ask the following question: 
Research Question 2.1 - How can seated light assembly workstation layout be assessed for system and well-being impact at the design stage?

The assumption of a small hand load in the developed tool necessitates the comparison with other hand loads with the impact on tool outputs determined by asking the following:

Research Question 2.2 - How do tool shoulder load moment outputs for different hand loads differ from the shoulder load moment outputs of the tool baseline models?

Asking the following two questions was needed to understand the potential user induced input error to the tool:

Research Question 2.3a - What is the average magnitude of error in hand location measurement?

Research Question 2.3b-Does the magnitude and direction of input error differ between measures taken from a virtual workstation and a matching physical workstation? (RQ 2.3a)

Investigating the impact of any user induced error in the tool was investigated in the following two questions:

Research Question 2.4 - How does the magnitude and direction of induced hand location input error affect a) predicted shoulder load outputs and b) estimated reach/move time outputs of the tool?

Research Question 2.5 - Using the results on the characteristics of hand location input error (RQ 2.3a, 2.3b) and the outcomes from testing induced measurement error (RQ 2.4), how large is the potential error due to the observed input measurement variability?

It is important to understand key components to the design of a usable tool. Understanding these factors not only benefits the current tool but have the potential to aid future tool development. As a result the following question was investigated:

Research Question 2.6 - What are the factors that influence a user-centered design approach to develop a human factors tool for predictive workstation assessment?

\section{Objective 3: Create a tool that reports the level of HF integration and maturity in an organization}

In the absence of an approach to assess how HF is integrated throughout and organization, the following question was explored: 
Research Question 3.1 - How can the level of HF capability and integration maturity in an organization be assessed?

Creating a valid tool necessitated the review of the tool with potential users. An examination of their views was as guided by addressing the following question:

Research Question 3.2 - What are potential user views on the contents of the tool developed in $R Q 3.1$ ?

The creation of a usable, field valid tool meant asking the following question in order to understand users' views:

Research Question 3.3 - What are users' views on the content and usability in a case field trial of the tool developed in RQ 3.1?

In Figure 2, the three objectives are related to the framework from Figure 1 to show how they relate to the general organization. The investigation of metrics in Objective 1 envelops the whole framework to demonstrate the review of a wide range of metrics and stakeholder views. The development of a design stage virtual human factors tool to assess a workstation in Objective 2 shows how the tool is applicable to design and development and contributes to leading, predictive metrics. Lastly, the organization assessment tool of Objective 3 is placed on the framework to demonstrate how its assessment capability spans an organization and assesses HF from a macroergonomic perspective. 


\section{Objective 1}

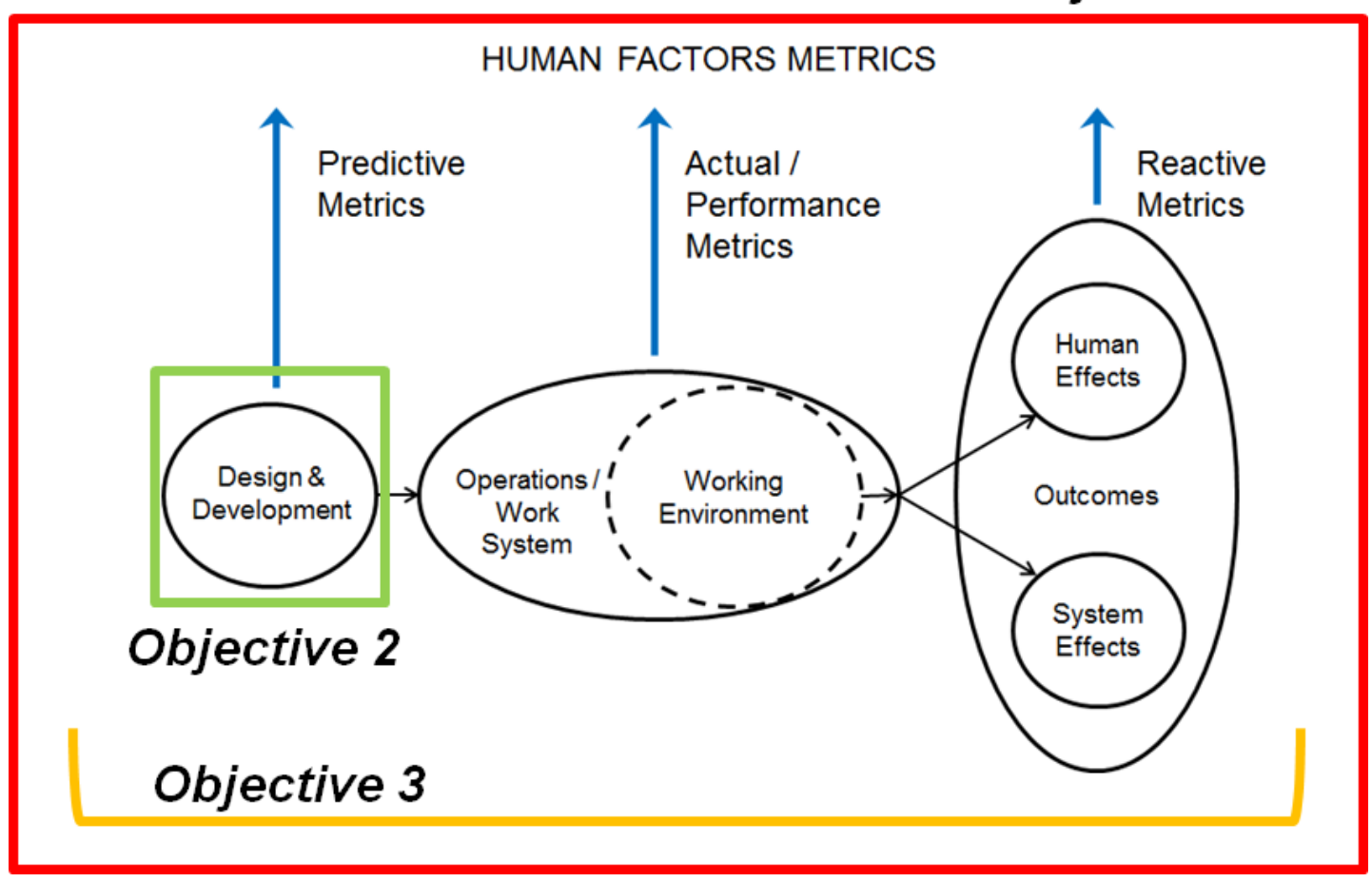

Figure 2. Relationship of dissertation objectives to framework outlined in Figure 1. 


\section{Chapter 2}

\section{General methods and research scope}

\subsection{Global project scope and methods}

This chapter describes the case site where the majority of the dissertation research took place and provides a description of the general methods for the entire dissertation. A more detailed description of the methods for each study can be found in the study paper in the specific chapter.

\subsection{Case site}

The case site is an electronics manufacturing company based in southwestern Ontario. The company designs, manufactures and provides service of their electronic devices for customers worldwide. During the time of the project the case partner underwent significant restructuring, shedding almost half of its global workforce by the end of the project, reducing from 16,500 employees globally to approximately 7,000 employees. This research was focused within the new product realization business unit of the organization. The unit was responsible for test building new devices and creating a manufacturing process to move to high volume manufacturing sites. In the early stages of the project the site also supported high volume manufacturing on demand and specialty product builds.

\subsection{Study Design}

This research is being conducted within a larger action research (AR), field research case project with an electronics manufacturer. The multi-year project examines the integration of human factors into production system design with the goal of improving worker and system related outcomes (see Village et al., 2014a for details). This case project uses mixed methods in an AR framework. AR is a cyclic process with components of planning, acting, observing and evaluating (Lewin, 1946). Researchers work in concert with the company personnel to reach a common goal and better understand the problem of interest. The interactive relationship 
between researcher and those being researched has been "characterised by joint action, joint involvement and shared responsibility” (Ottosson, 2003). In this interaction, researchers play less of a role of “expert”, transferring knowledge to their counterparts and instead take on a position where they engage with the knowledge of those in the setting (Neumann et al., 2012; Neumann and Village, 2012). The use of this approach allows for insight on change development from an embedded, close perspective (Neumann and Village, 2012).

Solving a problem for the research partner and contributing to science are two goals in AR (Ottosson, 2003). Mixed methods data collection means that both qualitative and quantitative information is collected and analyzed. In this case investigation, the mix of qualitative and quantitative methods varies depending on the study and which methods and information are most applicable to understanding and answering the research question. Some research questions, such as "Research Question 1.4 - What issues might affect efforts to develop and integrate HF metrics in the organization?”, are best addressed qualitatively while others, such as "Research Question $2.3 a$ - What is the average magnitude of error in hand location measurement?”, necessitated quantitative analysis. Qualitative information is predominantly field notes which include the description of interactions and researcher reflections. Audio recordings of discussions and interviews, surveys and company documents are some of the data gathered within the case study field notes. The project started with principal funding from the Workplace Safety and Insurance Board of Ontario in 2010 and supported two Masters students and two Ph.D. students. Other projects included a demonstration of the use of action research in the study of human factors integration (Village, et al., 2014a), process mapping (Lim et al., 2014), discrete event simulation (Dode, 2012), cognitive mapping (Village, et al., 2013a), tool development (Village, et al., 2014b), and theory development (Village et al., 2015).

\subsection{General methods for the case interaction}

Access to the case organization was initially through the health and safety manager who provided access to important stakeholders. Principle points of contact evolved with the project and the restructuring of the company. A steering committee oversaw and directed the research agenda for the whole project. The projects had interactions with over 100 members of the case organization and affiliated suppliers. Industry stakeholders had backgrounds including product design, production, quality, HF and health and safety. They included tooling engineers, project 
managers, production supervisors and managers, senior directors of associated work groups as well as HF specialists, and managers and personnel within environmental health and safety. A stakeholder was any individual who could contribute to the understanding, development or execution of a project or investigation in the case. Stakeholders were chosen based on the recommendations of other company stakeholders or if their contributions in associated work was observed to be relevant by members of the research team. Interactions with stakeholders occurred on a formal and informal basis. Formal interactions included interviews and workshops specifically targeting a focused topic. Informal interactions included general discussions, phone calls, email correspondence, and workshops or meetings where discussions on a topic naturally evolved within conversation. Stakeholder interactions were documented by field notes and/or audio recordings. Audio recordings were transcribed when information was relevant to the research. Field notes and audio transcriptions were gathered and analyzed within NVivo (QSR International Pty Ltd). More specific methods details can be found within each of the studies completed.

\subsection{Dissertation structure}

The dissertation objectives and associated research questions (RQs) are addressed within the studies presented in the dissertation as follows:

\section{Objective 1: Determine the company stakeholder views of HF metrics, metrics development and HF application}

Research Question 1.1 - How do HF metrics compare to engineering metrics in the organization?

Research Question 1.2 - How do stakeholders view metrics in the organization?

Research Question 1.3 - What factors affect how HF metrics relate to engineering stakeholders in the organization?

Research Question 1.4 - What issues might affect efforts to develop and integrate HF metrics in the organization?

Chapter 3 includes the paper "An examination of metrics in a production organization to identify factors affecting current and future human factors metrics.” This study investigated RQ 1.1 to 1.4 in order to develop an understanding of important issues for HF metrics development and implementation of future HF metrics and metrics systems. The case exploration was completed 
with co-authors Dr. Judy Village, Dr. Filippo Salustri, and Dr. Patrick Neumann. The candidate collected the data, completed the analysis and was lead author on the paper. This paper is in the final stages for journal submission.

\section{Objective 2: Develop a workstation level HF assessment tool for light assembly work}

Research Question 2.1 - How can seated light assembly workstation layout be assessed for system and well-being impact at the design stage?

Chapter 4 includes the paper " $A$ tool to predict human factors and work element times from workstation layout". This study reports on the development of a virtual HF assessment tool and demonstration of the application of the tool with a comparison to an observation based approach. The paper was completed with co-authors Dr. Judy Village, Dr. Filippo Salustri, Dr. Saeed Zolfaghari, and Dr. Patrick Neumann. The candidate developed the tool, completed the comparison analysis and was lead author on the paper. This paper is ready for journal submission.

Research Question 2.2 - How do tool shoulder load moment outputs for different hand loads differ from the shoulder load moment outputs of the tool baseline models?

Research Question 2.3a - What is the average magnitude of error in hand location measurement?

Research Question 2.3b-Does the magnitude and direction of input error differ between measures taken from a virtual workstation and a matching physical workstation? (RQ 2.3a)

Research Question 2.4 - How does the magnitude and direction of induced hand location input error affect a) predicted shoulder load outputs and b) estimated reach/move time outputs of the tool?

Research Question 2.5 - Using the results on the characteristics of hand location input error (RQ 2.3a, 2.3b) and the outcomes from testing induced measurement error (RQ 2.4), how large is the potential error due to the observed input measurement variability?

Chapter 5 includes the published paper "Testing of a workstation efficiency evaluator tool", which addresses RQs 2.2 to 2.5. This collection of three studies demonstrates the expected input error for users of the tool developed in Chapter 4 and the response characteristics of the outputs due to user error and variable hand loads. The paper was completed with co-author Dr. Patrick 
Neumann. Assistance was provided by Lindsay Buckingham (design of CAD models) and Shahriar Shahin (data collection) for the study assessing user input errors. The candidate developed the protocols, completed the analyses and was lead author on the paper. This paper can be found in: Greig, M.A., and Neumann, W.P. (2015). Testing of a workstation efficiency evaluator tool. International Journal of Industrial Ergonomics, 48, 60-69.

Research Question 2.6 - What are the factors that influence a user-centered design approach to develop a human factors tool for predictive workstation assessment? The conference paper "User-centered development of a workstation efficiency evaluation tool” addresses RQ 2.6 in Chapter 6. The study reported on the user contributions to the tool developed in RQ 2.1 and was presented at the Annual Conference of the Association of Canadian Ergonomists. The paper was co-authored with Dr. Shane Dixon, Tim Annett, Dr. Judy Village, and Dr. Patrick Neumann. The candidate collected the data and was lead author on the paper. Dr. Dixon provided contributions to the data analysis. The paper can be found in: Greig, M.A., Dixon, S.M., Annett, T.L., Village, J.L., and Neumann, W.P. (2013). User-centered development of a workstation efficiency evaluation tool. Proceedings of the 44th Annual Conference of the Association of Canadian Ergonomists, Whistler, Canada (October 8-10, 2013).

\section{Objective 3: Create a tool that reports the level of HF integration and maturity in an organization}

Research Question 3.1 - How can the level of HF capability and integration maturity in an organization be assessed?

Research Question 3.2 - What are potential user views on the contents of the tool developed in $R Q 3.2$ ?

Research Question 3.3 - What are users' views on the content and usability in a case field trial of the tool developed in RQ 3.1?

Chapter 7 presents the paper “Assessing human factors capability in organizations”. This study addresses RQs 3.1 to 3.3 and reports on the development and testing of a tool to assess the maturity of HF integration within an organization. The tool was collaboratively developed in the case organization and validated in workshops that contained subject matter experts from outside of the organization. The paper was co-authored with Dr. Judy Village, Dr. Shane Dixon, Dr. Filippo Salustri, and Dr. Patrick Neumann. The candidate spearheaded the development, collected and analyzed the data and was lead author on the paper. 


\section{Chapter 3}

\section{An examination of metrics in a production organization to identify factors affecting current and future human factors metrics}

\section{Introduction}

This chapter addresses Objective 1: Determine the company stakeholder views of HF metrics, metrics development and HF application and the following dissertation research questions:

Research Question 1.1 - How do HF metrics compare to engineering metrics in the organization?

Research Question 1.2 - How do stakeholders view metrics in the organization?

Research Question 1.3 - What factors affect how HF metrics relate to engineering stakeholders in the organization?

Research Question 1.4 - What issues might affect efforts to develop and integrate HF metrics in the organization?

Working to better understand the integration of human factors in production, this explorative case study with an electronics manufacturing company determines issues for HF metrics development. This chapter describes the views of stakeholders of metrics and identifies engineering and HF metrics. A qualitative review of the interactions is completed to assess for gaps in HF metrics and tools as they relate to the organization and to identify important factors for the development of HF metrics.

\subsection{Aim}

The aim in this chapter is to develop an understanding of important issues for human factors (HF) metrics which should be considered during the development and implementation of future HF metrics and metric systems. This aim will be addressed through a qualitative case investigation of an electronics company that will examine the following research questions (RQs): 
RQ 1 - How do HF metrics compare to engineering metrics in the organization?

RQ 2 - How do stakeholders view metrics in the organization?

RQ 3 - What factors affect how HF metrics relate to engineering stakeholders in the organization?

RQ 4 - What issues might affect efforts to develop and integrate HF metrics in the organization?

\subsection{Methods}

The textual material for this research was obtained from a longitudinal collaborative action research project in which new methods and approaches for integrating HF into production system design were developed in a case organization. The case organization is a large Canadianbased, electronics development, manufacturing, and service provider. The five year project was focussed primarily on the section of the company providing product development and realization. This chapter draws on these interactions and distills the information from the perspective of metrics.

\section{Study Design:}

The study is an explorative case study using qualitative data with multiple data sources from interactions with a variety of stakeholders. The project had interactions with over 100 members of the case organization and affiliated suppliers. Organization stakeholders had backgrounds including product design, production, quality, HF and health and safety. They included tooling engineers, project managers, production supervisors and managers, senior directors of associated work groups as well as HF specialists, and managers and personnel within environmental health and safety. Information obtained from formal and informal interactions was documented by field notes and/or audio recordings. Formal interactions included interviews and workshops specifically targeting metrics or related topics. Informal interactions included general discussions, phone calls, email correspondence, and workshops or meetings where discussions relating to metrics, measurement and information reporting organically evolved.

Formal and informal interactions were reviewed for metrics related information from 219 first author field notes, 89 phone calls, 137 site visits, over 360 hours of direct contact time, 106 hours of associated audio recordings and associated email correspondence, along with 47 field notes from other members of the research team. Field notes and audio transcriptions, completed 
as required, were gathered and analyzed within NVivo (QSR International Pty Ltd). Text was coded in inductive and directed approaches. All text was thematically coded based on the qualitative coding and the inductive analysis approach of Thomas (2006). Data was open coded with respect to information about metrics. Coded data was re-read to refine the focus of the code and similar codes were merged. Discussions throughout the data collection and analysis, with research team members engaged in the project and company stakeholders, provided clarity and verification of the interpretation of the data. Directed coding focussed on the identification of individual metrics, metrics sets and metrics system as defined by Melnyk, et al. (2004). Participant quotes presented in the chapter were adjusted for grammar and local jargon for improved clarity. Adjustments are noted using [square brackets].

\subsection{Results}

Interactions within the case organization are organized within four sections that correspond to each research question.

1) RQ 1.0 - How do HF metrics compare to engineering metrics in the organization?

This section illustrates the engineering and human factors metrics identified within the case organization and the how HF stakeholders were aware of the differences between the metrics of the two domains.

A variety of process and outcome measures were observed for engineering and HF metrics and indicators in the case organization. The engineering metrics could be clustered into four categories, leading, status, lagging, and response, shown in Figure 3. 


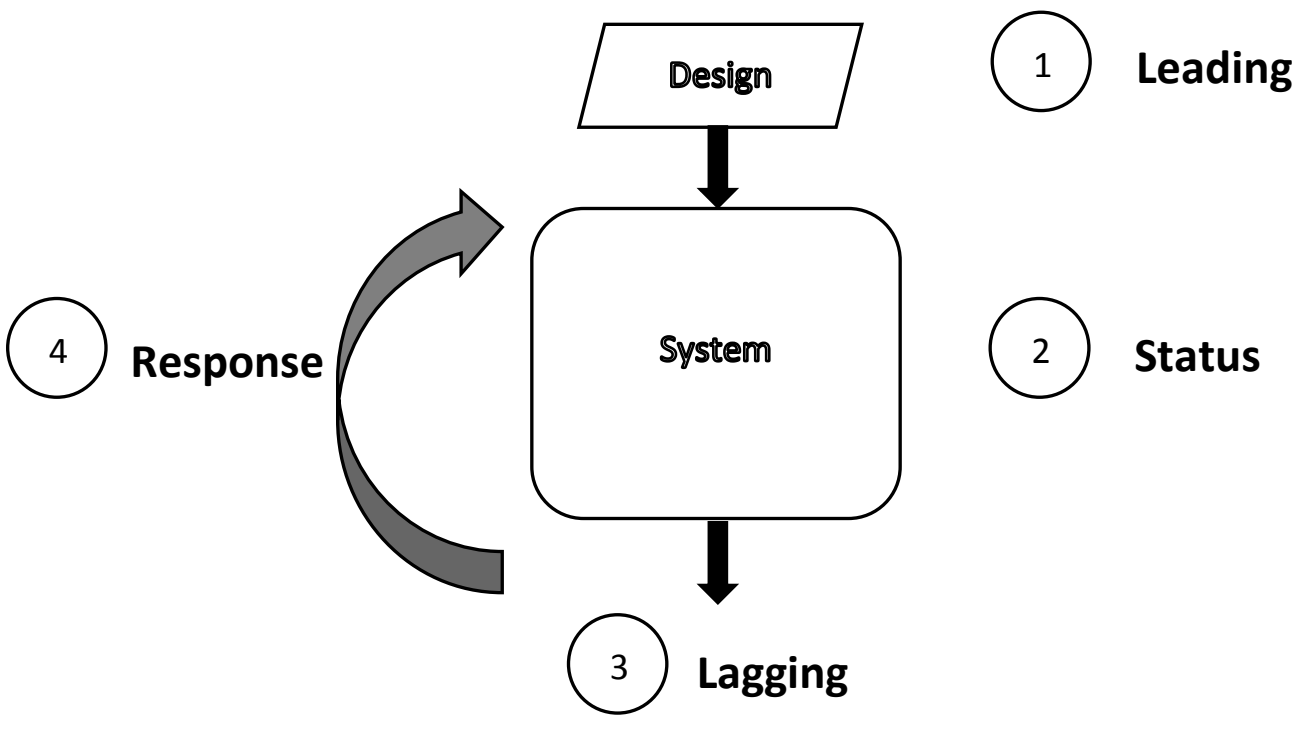

Figure 3. The four categories of engineering metrics and indicators that emerged from the review of the case organization.

These categories were defined for their reporting on the product design system and production system. The four categories of engineering metrics were defined as:

1. LEADING: future expectations for the production and product design systems (e.g. Engineering: rate, capacity, cost; HF: none applicable)

2. STATUS: reporting on the current state of the production and design systems with information on issues, inventory, and timing of events (e.g. Engineering: repair in progress, idle time, inventory; HF: number of ergonomic education days, risk assessments completed, training completed)

3. LAGGING: reflecting the outcomes of the production and product design systems and capturing information on productivity and quality (e.g. Engineering: defects, yield, scrap; HF: incident rate, injuries, force)

4. RESPONSE: reporting on what was done in reaction to an incident in the production and product design systems (e.g. Engineering: parts under investigation, corrective actions, initiatives; HF: time from request to assessment, physical demands descriptions completed)

HF metrics were clustered like the identified engineering metrics to examine similarities between the two. Compared to engineering metrics, few HF indicators were in the response metric 
category (e.g. physical demands descriptions completed, time from request to assessment). No applicable HF metrics were found that would fit into the leading metric category and relate to future expectations of the product design system and the production system. The greater HF representation was in the status and lagging clusters, due to the HF metrics reporting on injuries and how the HF group was completing their service of the organization.

An engineer expressed surprise when hearing of the lack of leading HF metrics in an early review of HF metrics in the local environment "I still don't believe this for a minute, that we don't have any human factors here or any indicators in our systems, right? I don't believe that, but that's what you guys are telling me so I need to believe that.” The lack of leading HF measures was not isolated to that engineer's design focused environment. The corporate directed performance metrics for the business unit and the re-designed supply chain measures did not include a significant HF presence, in part because of a lack of available information due to a new health and safety monitoring system that was still in development. A HF specialist stated "I saw the [group of performance metrics] come out and safety was not even anywhere close to being on there....... know this is a gap." In a later stage of the project, one HF specialist commented on the need to change the HF metric information when they stated that there was a "need to flip ergonomic thinking upside down”, in particular moving from reactive to proactive measures, and in their current situation a "need to move beyond ergo days", a lagging metric reporting on the occurrence of HF information events.

2) RQ 2.0 - How do stakeholders view metrics in the organization?

The concept of a metric was not always clear or consistent with every stakeholder or group involved. The term indicators and metrics were often used synonymously. An industrial engineer highlighted the need for term clarity commenting "Is an indicator a bullet point on a PowerPoint slide or is it a metric? A number? Is it a little happy guy that you stamp a happy face on because it is all good or is it.... a checklist?...I'm using indicators as the same as metrics..... [we should define] this is an indicator, this is a metric." In another conversation, discussing what makes a metric, the definition of a metric at the start was different from the one at the end of the discussion. Generally, a metric was identified as something measured that relates to the business and achieving success in the organization. One industrial engineer believed that " a metric should measure and make a difference to your existence. [It] should 
reflect the business" and added further that their "interpretation of the metrics" was that "Metrics drives behaviour. Drives system. Moves the company." A project manager in charge of revamping a group's key performance metrics spoke of metrics being related to targets, when they stated that there are "numbers that are set and we try to utilise those targets.... where targets didn't exist, the key stakeholders would establish what is it that they are driving to hit and that's the metric." The use of the term key performance indicators added another layer to the understanding of how metrics were defined. The key performance indicators term most commonly referred to a set of corporate directed tracking measures and included components that could be considered an indicator or a metric.

Engineers demonstrated a greater familiarity with metrics and indicators than the HF specialists in the early interaction discussions on metric development. One engineering manager commented that you "can't improve anything until we've got some metrics around." An HF specialist was "not really clear on metrics", although they did believe a good metric would be "something like a leading metric." Their struggle to contribute and develop metrics was captured when they stated "I don't know if I've helped so much on metrics....I found it kind of hard to come up with that metric." Around the time of this comment there had been extensive discussion with other engineering members of the research team around the goal of developing new HF metrics to report HF status within other aspects of the organization. The HF specialist went on to demonstrate progress in the understanding and development of metrics adding "We've started low and we're working our way up and eventually it'll turn into a metric."

3) RQ 3.0 - What factors affect how HF metrics relate to engineering stakeholders in the organization?

Engineering and HF metrics in the case organization highlighted the different goals and mindsets of the disciplines. From these differences a disconnection between HF metrics and engineering metrics was evident. Three themes contributing to the disconnection emerged. They include: a) HF reporting on health and health promotion which did not relate to the new product realization environment, b) engineering and HF operations silos reinforcing perceptions and knowledge of HF contributions, and c) HF specialists lacking tools to generate predictive measures. These themes are highlighted below. 
a. HF reporting on health and health promotion activities did not relate to the new product realization environment

Engineering metrics focused on productivity, efficiency, quality, production or product development and project management, all key components of a product realization centre. HF metrics were more focused on employee health information and the associated project management. HF information, in general, was noted by an industrial engineer to be “...semisecluded to our safety department... whatever event they are encountering, it's not being shared."

One HF specialist stated that "most [in the organization] don't see ergonomics beyond desks and chairs and injury prevention.” This sentiment was validated by a comment by an engineering director who "thought [the HF specialist] is a tables and chairs guy. I didn't understand how $[\mathrm{HF}]$ impacted our process." The default injury focussed mentality was frequently present, as evident in a discussion with an HF specialist who was reflecting on the "ergonomics side-car" phenomenon. The specialist commented how HF reporting needed to better align with the organization, saying how they need to "somehow get it into a metric that [others in the organization] understand." A lengthy list of guidelines that existed to aid work planning and workstation design similarly focussed on employee health and risk of injury. When information was quoted to engineers from these guidelines it was not always understood. For example, when an engineer discussed the negative physical impacts of contract stress, which was a guideline and HF work design item, the engineers appeared to not know how to handle the information. The impact of the information on their work was lost. The engineers' understanding changed once the context of the information was shifted to relate how the physical impacts would manifest to impact the ability of workers to do effective, quality work.

An HF specialist stated the current reactive injury and discomfort measures and reporting on 'ergo visits' “doesn't mean a whole lot and reflect everything that is done or [we're] trying to do to improve [the] ability for people to do work." The need to create a better connection was not lost on the HF specialists who suggested finding a way to link quality and HF would be useful, with one of them commenting "maybe we should understand the quality metrics so we can look at HF and design along the same line." Similarly, the desire to move beyond a health only contribution was evident when an environmental health and safety manager commented about 
wanting to a make a connection between a healthy, happy worker and successful product in the company.

b. Engineering and HF operation silos reinforces perceptions and knowledge of HF contributions.

In a discussion on task and workstation measurement and establishing HF metrics, the exchange between two engineers highlighted an ingrained thinking of the HF relationship to the organization. One engineer stated that "someone needs to be doing the measurement at a body level, if you like, and the other is how are we going to measure ourselves as a company." They believed metrics were more of a "higher level" and that at "the end of the day [measurement of the workstation] is work design. How are we going to score and measure ourselves at the corporate level based on this?” Similarly, the other engineer commented that "measuring ourselves as a company [and I] don't want to say that it's more important, but it's more important for the future" as opposed to examining an assembly step that might not exist in six months. For this engineer, metrics were more about having a "system in place to measure" that $\mathrm{HF}$ analysis was being done and less on measurement about "how do we get the job done...without the operator having discomfort." Further, the work at the workstation level was “more task oriented and metrics are more system oriented." Little credence was then given to the idea of HF metrics from work design from the employee perspective and that metrics instead should focus more on the "manufacturing execution of what we do. That's how we measure us as an organization.” When pressed further for how HF metrics would look at the corporate level an engineer responded "If I'm being completely honest I don't know what...I know how to go out and measure capacity across the global manufacturing sites across the first 10 sites. I can do that. We've got tools and models and scorecards to do that with. I don't know how to do that from a human factors perspective. That's where we're looking for help.”

c. HF specialists are lacking tools to generate predictive measures

An HF specialist in the case company was hampered by a lack of predictive measures of light assembly worker health and performance. They wanted the ability to score a job for the effect of moving work elements between workstations but found there is "no good way to score both [the] job or [the] task" in their situation. Most HF tools that were available to the human factors 
specialists to create an indicator or metric were manual material handling based and not suited for light assembly tasks.

4) RQ 4.0 - What issues might affect efforts to develop and integrate HF metrics in the organization?

Stakeholder interactions at the company identified factors for consideration when attempting to develop HF metrics and indicators. Themes that emerged focussed on: a) the knowledge of the audience involved and their knowledge of the metric information, b) the connection of metrics to the audience or organization goals, c) the support of the organization and from the organization, d) resource availability considerations, and e) the communication of information. Each theme is highlighted in the following sub-sections.

a. Knowledge of the metric audience and their knowledge of the metric information The theme of knowledge emerged to reflect how metrics developers need to understand the environment they are working with and how the knowledge level of the recipient of the metrics is important for understanding the metric. Knowing of key meetings, processes and deliverables could assist with creating relevant metrics. One HF specialist was unaware of when meetings occurred in the production development process but anticipated they could make a great contribution if they could get on the invite list. Likewise, HF specialists needed to understand the knowledge of those receiving the metrics feedback. For example, HF specialists highlighted that knowledge of what is behind the HF reporting numbers is a challenge when they stated that “[engineers] don't have our background and know why they need to understand the number. [It] doesn't mean anything to them." Comments from engineers supported this sentiment. One engineering director stated "too often you think of HF as CTS [carpal tunnel syndrome], not as [an] influence on quality." An engineering manager commented "Me, myself, I've been getting completely hung up on health and safety data. You know, what do we currently measure? I'm thinking injuries. Lost time. All of that stuff. But I now realize it's got an impact in everything that we do."

Another important aspect of knowledge related to the background knowledge about the metrics being used, meaning how the metrics were created and what they indicate. An engineer in a 
group revamping their performance metrics commented how they knew little of how the original values had been created. As a result, they had less understanding of the reasons, or the 'why', behind any undesirable 'red' issues that the metrics had indicated.

b. Connect metric content to the audience or organization goals

Connecting metrics to personal and organizational goals served to generate support for the metrics and make any added effort of obtaining the metrics more acceptable. Making a connection in the case organization could be done by determining which "measures can make their [managers'] jobs better." One industrial engineer commented that, the needs for metrics will be driven by the connection to organizational strategy. An engineering director demonstrated connecting to business strategy with the goal of evaluating situations in the organization when they said "we do all these things intuitively because we know it will show less defects, but we don't have an objective way of evaluating" adding further "if we had those ways of evaluating and comparing, then we can help the business make the right decision on how we prioritize our work. [It] comes back to prioritizing what's right for the business.” The mindset on how to connect to the case organization varied, understandably, depending on the stakeholder's role. One project manager commented on the need to have something that was proactive, reflecting company needs and providing the ability to "jump before [the customer] even said jump." The same project manager suggested that time, not cost, needed to be reflected. Conversely, engineers who worked in advanced manufacturing stated cost was very important. From the perspective of an engineer, and their supervisor, cost was "what I live by. People can tell you what great things they're doing but if I don't see the impact then I don't see it." Another engineer reflected more generally on the management interests as a way to connect stating that management "care about two things. They care about time/efficiency and quality. Both of those things link directly to dollars", and added further, "If you can show a [defect per million opportunities] with a particular process step and show that improving, it's something very tangible."

c. Support the organization and it will support the metrics

Management support for metrics was an issue discussed by participants. One industrial engineer stated "...everyone has to agree on these metrics for their linkages. The business case must be made for the metrics..... It's one thing for us to come up with ideas but if we amalgamate and 
report to [an engineering manager] or whoever and they don't agree then it won't stick. They have to agree." The engineer further commented that there is a "need to find the [metrics] that are most relevant" with "the direction to come from a higher level manager." The higher level direction ensures the metric "ties to a company directive/business directive" and is how the support of the business will assist with the business case for the resources required to create and maintain the metrics, value that can be determined by everyone agreeing on the metric linkages. A project manager furthered the idea when they said "if [a] production manager's boss [is] not asking [for] it, [it's] less likely it will work” and highlighted how senior level interests help to guide direction when they stated “Whatever my manager likes, I'm fascinated about.”

Gathering support was not always about solely getting the opinion from senior levels. A project manager reworking production supply chain performance metrics solicited support for decisions from a range of supervisors and managers. They thought that the multiple opinions would “converge" to a common view of what should be reported. Instead different expectations and wants "created more confusion" and made it challenging to merge everyone’s ideas to a common view. The project manager described the experience as “what one group manager thinks we should [have], maybe another area doesn't...what is it that we need to be measuring is all part of the discovery that we're still trying to figure out."

d. Resources are precious, so work within stakeholder constraints

Stakeholders commented on the concern for additional workload and encumbering processes. One engineer commented on the need to not bog down processes through the addition of a checklist, saying "things need to be done tout de suite”, or right away, to keep pace with a busy organization. Similarly, a project manager stated, “...we want to make sure that it is not adding any extra work....significant amount of work on the production floor or somewhere.” The potential of resource requirements of added work was not lost on an HF specialist who had received comments of “Don't bother me at quarter end. Don't bother me at month end” from colleagues. This HF specialist noted how the ease of use for new tools or new processes would be key to improve uptake and understanding, especially early on, commenting that "Initially it has to be [easy to use]. I think. And then build from there." They added further that the "metrics [are] something that is not a pain in the ass to get but metric[s] that work within what others are using.” Resources for people developing metrics can also be limited. At one stage in the project, 
time constraints imposed on one engineering manager to liaise with other production sites to improve operations had a greater immediate priority than making key performance indicators.

Minimizing added workload for metrics, but being too simple a process and generating too simple information, appeared to have a negative effect as well. In one example, a simple workstation design consideration checklist tool that had been developed with item rating from poor to excellent was considered a "soft" technique by one industrial engineer.

e. Communication with others should be in the language of their choice

After the development of a scorecard for human factors to support product and production design a product engineer commented how the scorecard brought things together and that they “otherwise wouldn't even know how to talk to you [the HF specialists].” The scorecard information had been developed with feedback and consideration of all relevant stakeholders to better suit the needs of the user. Interactions with stakeholders, in particular managers, found that tool output information was often desired in a style that provided a quick, high level view of the information, such as the top three issues to tackle. Presenting all of the detail had the potential to be overwhelming and lack an appropriate context to the end use. One of the HF specialists commented that detailed data and numbers are required but a checklist or symbol summary was the better method to present information to non-HF specialists. Their view matched those of engineers in that a simplified display of information was best, with the detailed data in reserve. Using a simple approach and providing clear information appeared to work well with engineers. One HF specialist believed that one industrial engineer would have desired a red/yellow/green indicator because "they clearly know what values they have to shoot for." As an industrial engineer commented, "engineers want to be told what to do." Further experiences with the organization demonstrated how one metric communication style does not work for all stakeholders. Opinions varied on the use of colours to represent a metric. A project manager noted that "red and green doesn't always apply, especially if it was not needed information" and that there was no need for it if a "manager was not asking for it and it doesn't relate to being best in class." An engineering manager and industrial engineer also shared the views that colours can lack detail and depth, "red makes sense, but what is the percent impact of red?" Similar to colours, symbols (e.g. O, X, $\Delta$ ) and ranking or scaling numbers (e.g. $0,1,2)$ were also 
present in the case organization's reporting, with product development engineers highlighting the importance of an appropriate scaling factor when ranking information with a simple scale.

The importance of communicating relevant numbers or something financial was highlighted by an engineering director lest raw numbers be "too nebulous, too touchy-feely for me."

Communicating qualitative information was also challenging in the organization. Collecting and presenting employee feedback, such as discomfort information, was one type of HF information that some may have considered relevant but not all agreed. Employee opinion was deemed untrustworthy and unscientific according to one industrial engineer. From their perspective, “That's people's opinion. What we're looking for is science based solutions here not someone's opinion." Part of the hesitancy towards opinion based information was that when "you ask people and you start to get expectations...it's a big can of worms and sets the expectation that the company is going to do something because they have information now." With this view in mind, it is understandable that attempts to collect and present employee discomfort information in the organization were usually unsuccessful.

\subsection{Discussion}

This explorative case study examined metrics from a HF perspective in a production organization. Other research has examined metrics in a production organization, for example studies highlighted in a review by Gomes et al. (2004) or work by Godener and Söderquist (2004) or Gosselin (2005). To the best of the authors' knowledge this has not been done before from the HF perspective. The findings in the case organization supports frameworks (Jensen, 2002; Neumann et al., 2009; Neumann and Village, 2012) that show HF information is dissociated from other organizational measures and does not readily contribute to design and strategy level decisions.

Stakeholder views of metrics were consistent with previous findings, showing that the metrics term is used inconsistently, which can cause confusion (Melnyk, et al., 2004; Huwe, 2010). Developers of HF metrics thus need to be clear with how the audience interprets the term metric. An individual metric has been defined as a quantitative or qualitative measure compared to a reference point but the term can also represent a set of metrics or the performance measurement system (Neely, et al., 1995; Melnyk, et al., 2004; Keong Choong, 2013). The identified purpose of metrics, to guide and drive behaviour, was consistent among engineering stakeholders. 
Engineers were more familiar with the metrics concept which may be a reflection of engineering processes being more tightly coupled with core business activities in the case organization. HF specialists were less familiar with metrics, especially beyond the health and safety focus that is common for HF specialist work (Theberge and Neumann, 2013). HF specialists need to be more aware of metrics principles and how HF can contribute to this management aspect of organizational operations.

The type and focus of HF and engineering metrics in the case organization were not surprising. Few HF measures are in use in manufacturing organizations or mentioned in professional practice and academic literature (Gosselin, 2005). Engineers in the case organization were key drivers of product and production design, which the metrics reflected. HF measures were stereotypical of HF focus on health and safety and quality of work life (Kleiner, 2004). The HF measures observed were typical based on HF tools used by HF practitioners (for examples see Wells et al., 1997; Dempsey et al., 2005; Neumann and Wells, 2007; Pascual and Naqvi, 2008; Takala, et al., 2010). A commonality across these tools and their outputs is that they are generally disconnected from organizational processes. HF measures for productivity, performance time and errors reflect organization processes and are also part of traditional HF measures identified by Kleiner (2004). These types of measures were not initially found in the case organization but evolved later in the study through other projects (see Greig et al., 2013a; Village, et al., 2014b). The concentration of reactive HF metrics and indicators reflected the reporting on injuries and activities, both used to justify HF practices and contributions in the organization. HF specialists commented on the need to change the focus of their reporting metrics to better represent performance and well-being. More performance reporting and proactive measures would help to bridge the gap between HF specialists and engineers. Currently, appropriate HF metrics are lacking (Neumann, et al., 2013) leaving the dual HF goals of performance and well-being (Dul, et al., 2012; International Ergonomics Association, 2014) underrepresented.

Three factors appeared to affect how HF metrics could contribute to engineering metrics in this case: 1) the observed health focus of HF; 2) the separation of HF and engineering; and 3) the lack of predictive HF tools. The health focus and organization location of HF specialists away from engineers was not unusual in industry. HF specialists typically play a health and safety 
supporting role within human resources and/or health and safety departments (Theberge and Neumann, 2013). Spanning the organization structure thus necessitates organizational work to justify ergonomics (Theberge and Neumann, 2010) and tailoring work assessments to convince the target audience (Wells et al., 2013). The ability to make these connections is dependent on HF knowledge and resources available in the company. Early in the research project, engineering stakeholders were unaware of the capability of HF to improve productivity and quality. HF specialists were without metrics that were relevant to engineers. Further, the HF specialists used design guidelines that were health risk factor based making the guidelines difficult to relate to the organization performance goals of engineers and managers. The healthfocused information combined with the differences in understanding of HF capabilities prevented HF metrics from contributing to engineering metrics. However, changing the context of the HF information to better align with engineers can help. Communicating HF information in an engineering context can improve HF integration into design processes and facilitate HF training for engineers (Broberg, 2007; Village, et al., 2013b; Hall-Andersen and Broberg, 2014; Village, et al., 2014b). As this project progressed, the understanding of avenues available to connect HF to the organization improved at the workstation level (see Village, et al., 2013a; Village, et al., 2014a; Greig et al., 2015). This included the development of HF tools that integrated within engineering tools and allowed light assembly workstation assessment (see Village, et al., 2014b) and assessment of HF maturity in the organization (Greig, et al., 2015). These tools enhanced the HF specialists' metrics capabilities and addressed one engineer's concern about the ability to score themselves at an organization level. Previously, the HF specialists in the organization were limited in workstation assessments by mostly observationbased reactive tools that did not relate well to light assembly workstations in a product and production design environment. Proactive tools combining performance and well-being information did exist (e.g. ErgoSam - Laring, et al. (2005), Ergo-MTM - Caragnano and Lavatelli (2012)). However, these tools were more appropriate for manual materials handling or automotive assembly than the electronics assembly in this case. The tool development in the case research project and the tools that were available for HF specialists to use highlighted the need for HF tools and indicators which are not exclusive to the knowledge of HF specialists, especially if non-human factors specialists are to use and apply HF in design. 
The metrics related issues identified in this HF focussed study are similar to those highlighted in other more general studies of metrics and performance measurement. Critical factors for performance measures include having measures that are relevant to the business, clear and simple, and cost-effective (Franco and Bourne, 2003). Characteristics of performance measures summarized by Hudson, et al. (2001) speak to connecting to the organization strategy and low resource requirements through ease of use and quick feedback. Resource availability, in the form of time and effort has also been shown to factor in organizational performance measurement implementation (Bourne et al., 2002). Factors enabling strategic performance measurement systems are also multifactorial (Franco and Bourne, 2003). The critical factors similar to this study include alignment between business and strategic measures, management understanding (including training), communication and reporting style, and management leadership and commitment (Franco and Bourne, 2003). Management support has been a contributing factor to measurement program implementation with the support changing as implementation proceeds (Bourne, et al., 2002; Bititci et al., 2006; Mendibil and MacBryde, 2006). Similarly, management support and resource time and effort commitment are necessary for successful participatory ergonomics program development (Jensen, 1997; Haims and Caryon, 1998; Driessen et al., 2010; van Eerd et al., 2010). Relationships with key stakeholders, which includes management support, has been suggested as needing to be developed to improve the prospects of HF influence on system design in the organization (Dul and Neumann, 2009; Dul, et al., 2012). This study has demonstrated the need for the resource connection from the context of HF metrics. Although the issues affecting metrics development in this study are identified as isolated themes they are likely to interact, which could impact HF metric development. Factors highlighted in the implementation of team-based performance measurement systems have been shown to interact both positively and negatively (Mendibil and MacBryde, 2006).

As a whole, the results of this study show the importance of knowing and understanding stakeholders involved with HF metrics. Understanding the interests of the metrics audience could help to create metrics with a greater perceived usefulness and ease of use, two factors that have been shown to improve technology acceptance (Davis, 1989; Davis et al., 1989). Having stakeholders contribute from different levels of the organization may help to better connect metrics capabilities at the micro-organization level with macro-organizational interests. Stakeholder and user input is commonly promoted for performance measurement design, 
strategy implementation (Platts, 1994), understanding design requirements (Dym et al., 2005), and to help create objectives and measures in the design of performance measurement systems (Bourne, et al., 2000). This suggests that HF metrics need HF principles and methods applied to their development. Participatory ergonomics (e.g. Haines et al., 2002) and user-centered design (e.g. Eason, 1995) are approaches that could be used to develop HF metrics. Process mapping (Lim, et al., 2014) and cognitive mapping (Village, et al., 2013a) could also compliment participative approaches as they have been applied to better understand engineering processes and the links between HF and organizational goals. Macroergonomics, which has an organization level focus, could help to relate metrics to different aspects of an organization since it provides a sociotechnical approach that captures the larger system perspectives of HF (Kleiner, 1996; Kleiner, 2004).

Based on the results of this case study, the following recommendations for HF practitioners interested in developing HF metrics seem warranted (Table 1). 
Table 1. Recommendations for developing HF metrics based on study results. Contributing results are indicated by R\#-p.\#\#, where \# corresponds to the results section and \#\# the page where the result can be found.

\begin{tabular}{l|l}
\hline \multicolumn{1}{c|}{ Recommendation } & \multicolumn{1}{c}{ Supporting Results } \\
\hline $\begin{array}{l}\text { 1. Understand other organization processes } \\
\text { and identify key milestones to support. }\end{array}$ & $\begin{array}{l}\text { Engineering metrics are focussed on different } \\
\text { goals compared to HF metrics (R1-p.35). } \\
\text { Know what information is important, and } \\
\text { when the information is important and most } \\
\text { relevant to the audience. }\end{array}$ \\
$\begin{array}{l}\text { Knowledge of the metrics audience (R4a- } \\
\text { p.41). }\end{array}$ \\
$\begin{array}{l}\text { the target audience. } \\
\text { Views of metrics and metrics definition differ }\end{array}$
\end{tabular}

Determine their understanding of metrics and knowledge of the information that you are able to share.

3. Educate the audience on the benefits of HF.

Provide education as needed using audience and organization relevant examples. Simultaneously highlight $\mathrm{HF}$ as a performance and well-being benefit.

4. Be relevant to strategic goals or goals in the organization.

Knowledge of the metrics audience (R4ap.41).

Engineering and HF silos reinforces unhelpful perceptions and knowledge of $\mathrm{HF}$ contributions (R3b-p.40).

Audience knowledge of metric information (R4a-p.41).

Connect metric content to audience or organization goals (R4b-p.42).

Move HF beyond a health and safety focus and demonstrate HF as a means to system and organization success. Identify key stakeholders for support and gain their support by connecting to their personal or organization goals.

5. Work within existing processes and minimise additional work.

Support the organization and it will support the metrics (R4c-p.42).

Create metric processes (e.g. HF tools) that integrate within existing work flow or add minimal work. Identify appropriate timing to introduce new concepts and demonstrate the importance to their role or the organization.

6. Create information in a style that is familiar to the audience.

Determine if the audience prefers colours, symbols, or data so that HF information is in a more familiar format and integrates within their traditional thinking.

Support the organization and it will support the metrics (R4c-p.42).

Resources are precious so work within stakeholder constraints (R4d-p.43).

HF reporting on health and health promotion activities did not relate to the new product realization environment (R3a-p.39).

Communication with others should be in the language of their choice (R4e-p.44). 
Methodological Issues:

This explorative investigation was limited to a case study of a light assembly product and production design operation. The exploratory nature resulted in a heterogeneous, diffuse data set with many stakeholder viewpoints considered. The breadth of data has helped to identify a range of factors for further investigation. More research is necessary to determine the generalizability of the current findings. Future work should investigate the requirements of HF metric development in other industries. Work is also needed to understand and create key metrics to bridge the gap between engineering metrics and HF metrics. A part of this future research could also include investigating the influence of organizational culture on the integration of HF into organizational performance systems.

More HF tools are also needed to build the measurement capabilities assessing HF impact on employee performance. A better understanding of the HF and system connections, such as quality and productivity, is also required to be able to address the gap. Developed tools should also consider a range of stakeholder interests to both improve information relevance to stakeholder processes and improve the potential for tools to be taken up and used. Establishing sufficient HF base measures can lead to future work investigating HF metrics sets as they relate to different organizational focuses and frameworks for a HF metrics system.

\subsection{Conclusion}

This exploratory case study investigated HF metrics in an electronics product development and production organization to better understand important factors for HF metric development and implementation. Engineers were more familiar with the concept of metrics but had varying interpretations of the definition of metrics. HF metrics were reactive, less in number and of a different focus compared to engineering metrics across four identified categories of leading, status, lagging and response. There were no HF leading metrics that could contribute to product design systems or production systems. The gap between engineering and HF metrics was due in part to the health and safety focus of HF in the organization, the physical location in the organization and knowledge separation of HF specialists and engineers, and the lack of predictive HF tools to create metrics. Issues affecting HF metric development identified five themes for to consider when developing HF metrics, including: 1) knowledge of engineer 
processes and of HF principles, 2) connection of metrics to the organization, 3) support of the organization and of the information to the organization, 4) resource availability and limitations, and 5) communication format of metrics information. From the results of the study six recommendations were created for HF practitioner interested in developing metrics. Though results come from an exploration in a case organization, these findings provide initial points for consideration in the development and implementation of HF metrics. 


\section{Chapter 4}

\section{A tool to predict human factors and work element times from workstation layout}

\section{Introduction}

To understand objective 2: Develop a workstation level HF assessment tool for light assembly work, this chapter addresses the following dissertation research question:

Research Question 2.1 - How can seated light assembly workstation layout be assessed for system and well-being impact at the design stage?

From interaction with the industry partner a need was identified to be able to assess human factors (HF) at the workstation level. This chapter describes the development of a novel tool for seated light assembly that predicts HF and work element times from the task descriptions of a workstation layout.

\subsection{Aim}

The aim of the chapter is to present the development of a novel virtual human factors tool and demonstrate its capabilities with a case example. The chapter describes how the tool predicts human factors and work system information using inputs from an existing layout, or proposed CAD design, of a seated, light assembly workstation. The case example will demonstrate the application of the tool using information from a workstation redesign assessment conducted by Neumann, et al. (2002) to illustrate how work cycle and shift demand requirements can be reviewed in the design stage. 


\subsection{Tool Development}

\subsubsection{Vision for the tool}

The goal of the tool is to provide a simple, predictive method, requiring no specialised ergonomics knowledge to assess worker shoulder loading, hand movement, and task time requirements in light assembly.

\subsubsection{Tool structure}

Input information for the tool is obtained from information describing a light assembly workstation, where from a seated position the worker reaches for all supplies and to complete all tasks without leaving or rotating in their seat (see example in Figure 4). The spreadsheet based tool uses inputs describing the three dimensional location of the hands for each task element, the type of hand movement and action required, and the gender specific population percentile anthropometry to be assessed. Inputs can be determined as early as the digital drawings in the design stage. Outputs include the estimation of shoulder load, hand travel and time of completion for the listed task elements. From the output information, more effective layout and assembly sequence decisions can be made with greater cost effectiveness and reduced risk exposure to the worker. The general logic of the tool is shown in Figure 5.

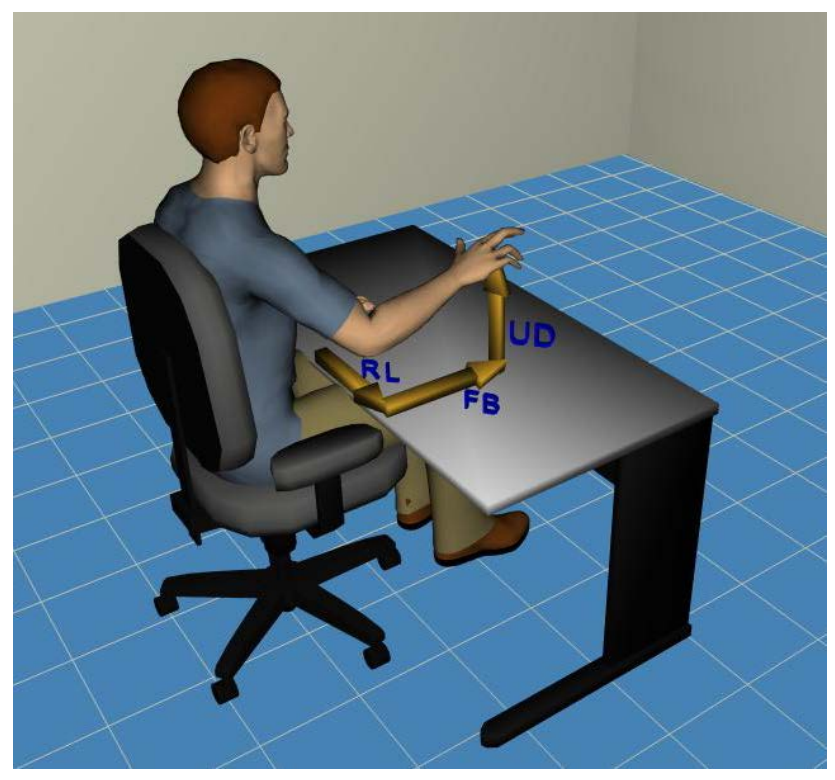

Figure 4. Measures required to describe worker hand location, where: RL is the right-left distance, FB the forward-backward distance, and UD the distance up from an origin at the top, leading edge of the workstation in line with the midline of the worker. Positive values match the direction of arrows. 
Inputs

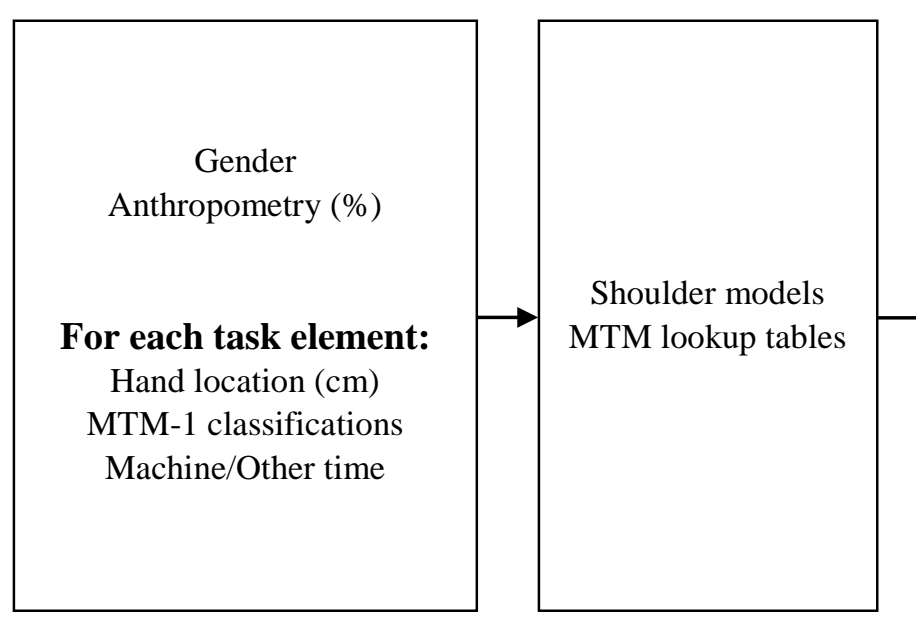

Outputs

\section{Human Factors}

Reach locations with zones of acceptability

Hand movement distance $(\mathrm{cm})$

Cumulative hand movement distance $(\mathrm{cm})$

Net Shoulder Load (Nm and \% MVC)

Cumulative Net Shoulder Load (Nms)

System

Task Movement and Process time (s)

Total Task time (s)

Cycle time (s)

Figure 5. Logic flow of the tool. Where \%MVC is the estimated shoulder load as a percentage of the maximum capability for a chosen gender and anthropometry.

\subsubsection{Tool inputs}

Inputs include setting the gender, the specific population percentile anthropometry $\left(5^{\text {th }}, 50^{\text {th }}\right.$, or $95^{\text {th }}$ by combined height and weight), and a description of the work task element sequence for the work cycle. The task elements include any reaching, getting, placing and machine operation steps. This level of detail is required for the prediction of time. Input data for each task element comes from two main sources, task element location and the Methods-Time Measurement (MTM-1) based element classifications (cf. Freivalds and Niebel, 2009). The contribution of the hand locations, population percentile and task classifications to the tool outputs will be explained in further detail in the next section.

Task element hand location, in centimetres, is the key driver of the tool as it is used for hand movement calculations, illustrating task reach zones, providing the inputs to the net shoulder load models and, when coupled with the element classification, driving MTM-1 reach and move 
time prediction. Hand location values are the three dimensional coordinates of the hand location for a work element. For a given element, the location may represent the average hand location or the worst case scenario posture to establish risk boundaries. The distances in each dimension are relative to an origin on the front, top edge of the workstation opposite the midline of the worker (Figure 4). Positive values are to the right of the origin, up from the workstation surface and moving forward, away from the worker (see Appendix A for an example of the input table and task values). This origin location allows the hand location inputs to be measured independently of workstation height from the floor and independently of the body size of the worker.

Connecting the hand location to different anthropometries, to allow shoulder load prediction, requires an assumption of a standard sitting posture and a translation of the origin to be relative to the worker.

Task element time prediction requires the analyst to identify the element as a reach or move, grasp or release, and the positioning required for any placement within a task (see example in Appendix A), according to the standard for MTM-1 (cf. Freivalds and Niebel, 2009). Choices are made through options available in drop-down menus. Eleven distinct MTM-1 grasp classifications and two different release choices are available to describe the method of obtaining or letting go of an object. The positioning required to place an object is described by one of nine descriptions. Any tasks without an MTM classification (e.g. machine time, inspection time, running a screwdriver) can have a time value, in seconds, entered manually.

\subsubsection{Tool processing and outputs}

Output information from the tool is organized into four categories: reach zones, hand travel distance, time estimation, and net and cumulative shoulder load. The processing of the input data to create each output value is explained in detail in the following sections.

\subsubsection{Reach zones}

Hand locations are directly mapped onto side and overhead view digital representations of workstations that include reach zone guidelines. The reach zones assist with the assessment of worker risk and help target task elements for continuous improvement. The overhead view zone 
acceptable for frequent reaches is defined by a $25 \mathrm{~cm}$ radius arc from the workstation edge, directly in front of the right and left shoulders, whereas an occasional reach, or cautionary zone, is bounded by an arc of $50 \mathrm{~cm}$ radius (adapted from Canadian Standards Association, 2012). An acceptable, frequent reach zone is defined in the side view by vertical heights under $15 \mathrm{~cm}$ from the top of the workstation surface (adapted from Putz-Anderson, 1988). The cautionary, occasional reach zone is bounded by the shoulder height of a $5^{\text {th }}$ percentile female, since working above shoulder height has been highlighted as an increased risk to the worker (Bernard, 1997; Punnett et al., 2000; Svendsen et al., 2004), and effective design should include as much of the working population as possible.

\subsubsection{Hand travel distance}

Hand travel distance, in centimetres, is calculated by the three dimensional straight line distance between corresponding task element hand locations. This novel indicator has been created as a generic workload indicator. It tracks movement distance throughout the workstation similar to tracking how far a worker walks around their work environment. The travel distance information, assumed to be linear between elements for calculation purposes, is also used to help predict movement time between consecutive elements. Cumulative hand travel is a summation of all of the hand travel distances to determine the total hand travel for the set of work elements for the right or left hand. A representative output of data from the tool is displayed in Figure 6. The hand travel contributions of each task (shown by columns) to the total hand movement (shown by lines) can be seen, and task specific changes can be targeted to assist continuous process improvement while managing worker risk. 


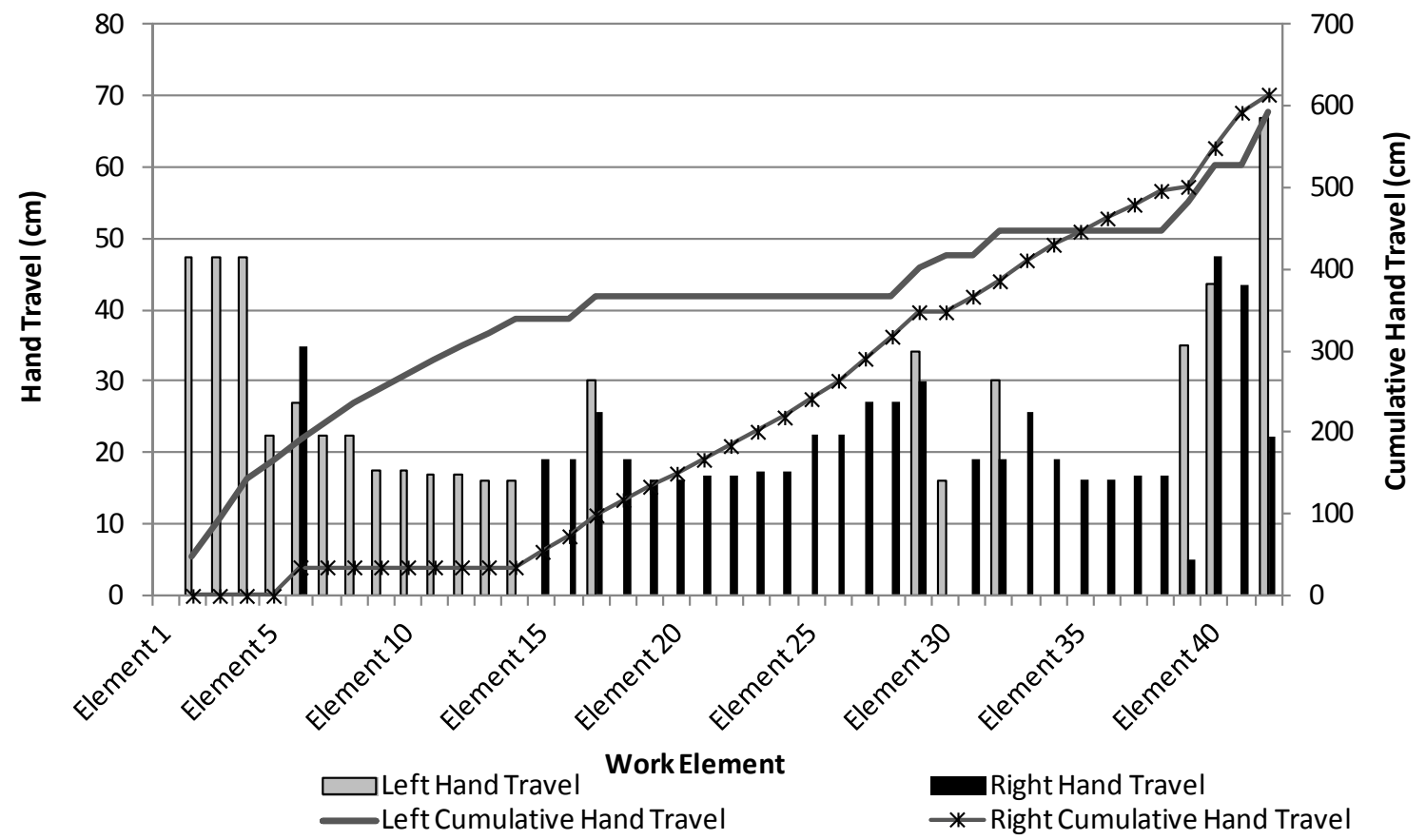

Figure 6. Example output summary of a set of work elements for cumulative hand travel and between element hand travel for the right and left hand. Number of element labels reduced to improve clarity.

\subsubsection{Time estimation}

The sum of the individual element times generates an estimation of the total work cycle time required for a normal, trained worker. The calculation of the movement, process, task, and cycle time are described in more detail in the following sub-sections.

\subsection{Movement time}

The hand movement distance between consecutive task elements is compared with the MTM-1 reach/move classification lookup tables to determine movement time. Time is presented in seconds in the output. Distances that are between levels in the lookup table are rounded up to the next level, meaning time is rounded up and not down. The MTM-1 'C or D' (reach) and ' $\mathrm{C}$ ' (move) Case and Description categories for small objects, accurate grasp and moves to an exact location, are used to predict time for all of the reach and move tasks (Freivalds and Niebel, 2009), since light assembly tasks require accurate grasping to pick up and accurately place potentially small objects.

\subsubsection{2. $\quad$ Process time}

Process time, converted and displayed in seconds, is a function of the two different types of classifications - grasp/release and position class of fit. The selection from the drop-down menu 
options in the spreadsheet is used to identify the corresponding time value from the MTM-1 tables (Freivalds and Niebel, 2009). The value is converted to seconds and displayed for each element.

\subsection{Element and cycle time}

Element time is the sum of the right and left hand movement times to get to the element location, the right and left hand process times while at that location, and any machine/other time. As required, the left and right hand actions are combined using prescribed MTM logic for simultaneous actions (cf. Freivalds and Niebel, 2009). This logic allows movements, but not processes, to be completed simultaneously. As a result, in simultaneous hand movements between successive elements, the larger time for the left and right hand movement time is used. Total cycle time is the sum of the individual element times. A representative output of element times in a work cycle can be seen in Figure 7.

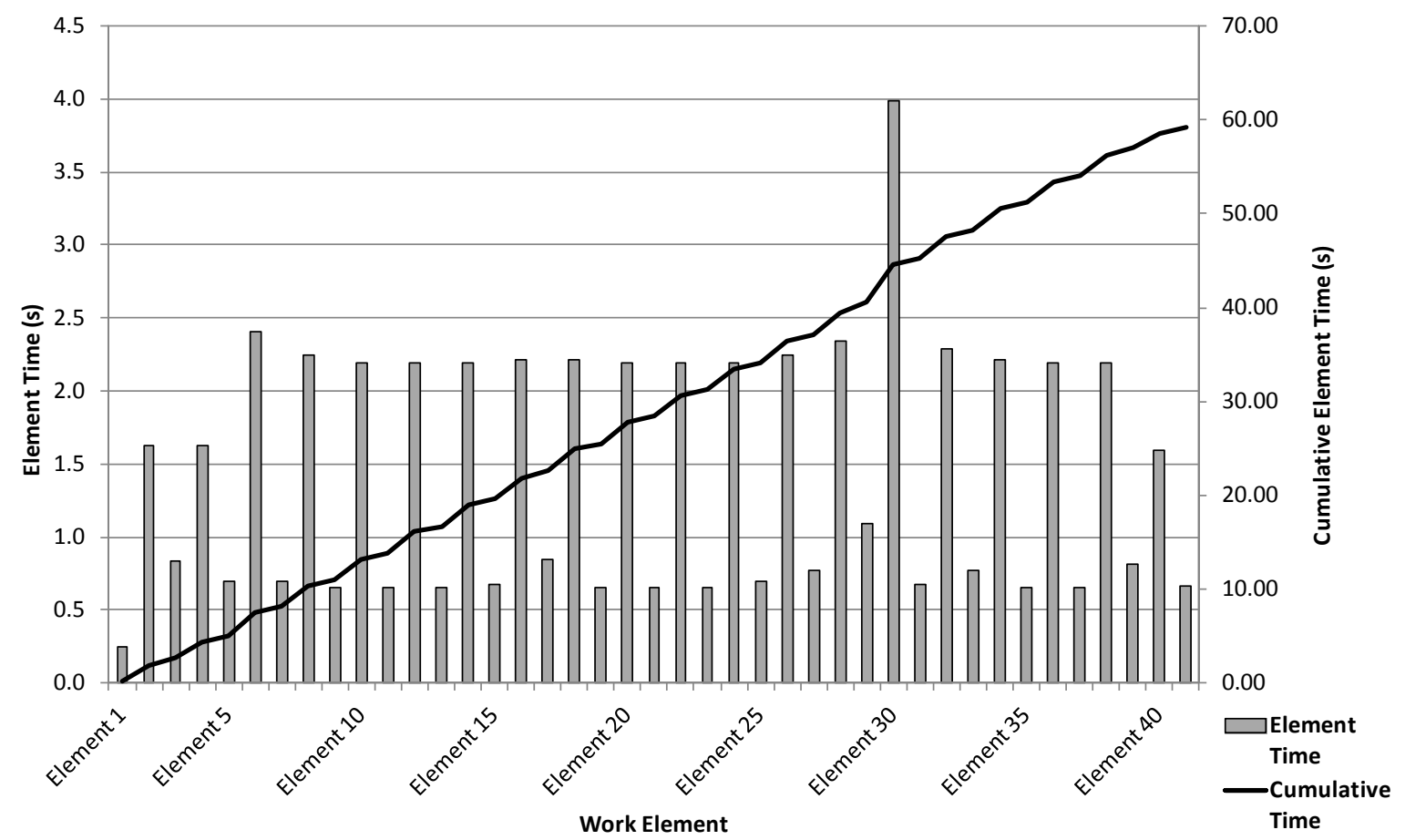

Figure 7. Representative graph showing individual element time contribution to the cumulative work cycle time. Number of element labels reduced to improve clarity.

\subsubsection{Net and cumulative shoulder load}

For each shoulder, two net shoulder load values, an absolute (Nm) value and a value relating the absolute value as a percentage of the capabilities of the population (\%MVC) being assessed, are reported for each element along with a cumulative load value (Nms) for the full list of elements. 
These measures were chosen to monitor known risk factors for musculoskeletal injury (Bernard, 1997; Norman et al., 1998) and to understand the load relative to the strength capability of the anthropometry being examined. Obtaining the net shoulder load values from the tool inputs requires the use of two separate shoulder models that have components dependent on the population percentile chosen and the input element location values, which are translated to an origin to match the input requirements of the shoulder models. The cumulative net shoulder load value combines the absolute net shoulder load value with the corresponding individual element time across the work cycle. The development of the models and outputs will be expanded upon in the remainder of this section.

\subsection{Development of shoulder load models}

Absolute and relative shoulder load prediction models were created as a function of hand location using data from a two dimensional digital human modeling software program (4D WATBAK, Version 2.0.37 - Ergowatch, University of Waterloo, Canada; Neumann et al., 1999). Data values were obtained by sequentially positioning the arm of the mannequin at $30^{\circ}$ intervals of shoulder flexion from $0^{\circ}$ (arm down at the side) to $150^{\circ}$ (arm near vertical over the head). Within each shoulder angle position the forearm was positioned at $30^{\circ}$ intervals from $0^{\circ}$ to $120^{\circ}$ (see Figure 8). The shoulder load for each position was also converted to a percentage of the population average maximum and termed as a percent of maximum voluntary capable (\%MVC) using values from within the software (cf. Neumann, et al., 2002). Data sets were compiled for the default male and female $5^{\text {th }}, 50^{\text {th }}$, and $95^{\text {th }}$ percentile included in $4 \mathrm{D}$ WATBAK. The heights and weights within 4D WATBAK were based on 1981 Canada Fitness Survey with limb segment information based on the works of Dreyfuss (1966), Plagenhoef (1971), and Zatsiorsky and Seluyanov (1983). No hand load was used with the mannequin in the initial model due to the low external loads anticipated in light assembly - for example, as seen in small electronics assembly, and due to the knowledge that the hand could be unloaded for a significant part of the work cycle while the worker reaches for a part or tool. Further, the part weights, up to a few hundred grams, are a small percentage of the worker's limb weight being moved about the workstation. With these assumptions, a hand load value is not needed as an input to this proof of concept version of the tool and the associated shoulder models. 
Three data sets were created from the digital human model data for each of the male and female population percentiles. These included Full Range (all data - see Figure 8, picture A), Above Shoulder (maximum shoulder flexion angle 90 degrees- see Figure 8, picture B) and Below Shoulder (no limb position or hand location above the level of the shoulder - see Figure 8, picture C). Information for the Above Shoulder data set is presented within the chapter since it was most relevant to the initial application of the tool in seated, light assembly. Model development was the same for all three data sets. The information for models of the remaining two data sets is presented in Appendix B for those readers that may have an application for it.

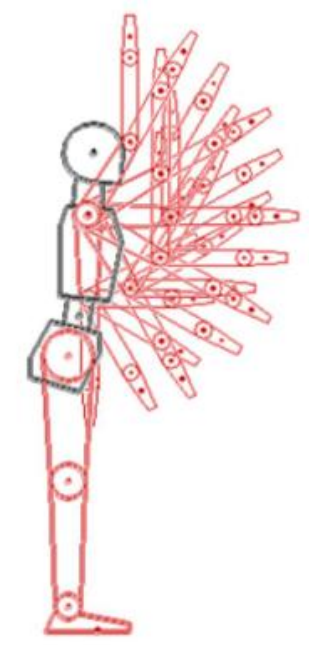

(A) Full Range

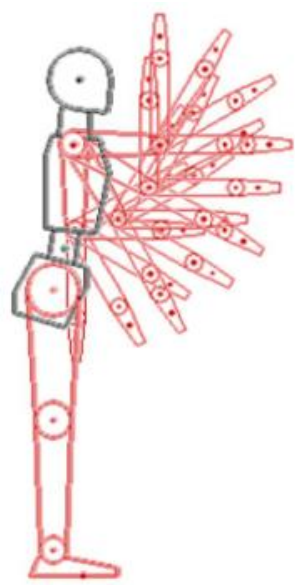

(B) Above Shoulder

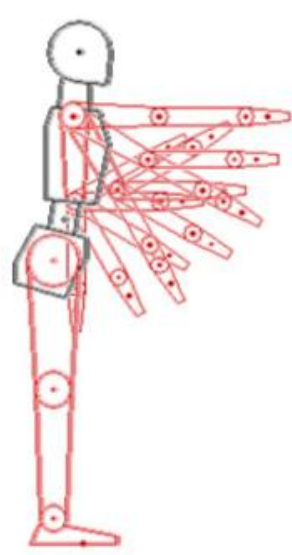

(C) Below Shoulder

Figure 8. Arm positions used for the three data sets.

For each data set, two linear regression models were generated in Microsoft Excel, one each for the shoulder load and \%MVC. Both models were functions of two input variables, the horizontal and vertical location of the hand relative to the shoulder.

The equations generated are shown in Table 2 and correspond with the generic shoulder load equations below. Equations for the other data sets can be found in Appendix C. Regression $\mathrm{R}^{2}$ values ranged from 0.86 to 0.88 for shoulder load and 0.81 to 0.82 for \%MVC. The range of $\mathrm{R}^{2}$ values for all models tested can be seen in Appendix C. 
Net Shoulder Moment ( $\mathrm{Nm})$

$$
\begin{aligned}
& =\text { Intercept }+X \text { Coefficient } \times x\left(\text { hand }_{(m)}-\text { shoulder }_{(m)}\right)+Y \text { Coefficient } \\
& \times y\left(\text { hand }_{(m)}-\text { shoulder }_{(m)}\right)
\end{aligned}
$$

Net $\% M V C(\%)$

$$
\begin{aligned}
& =\left(\text { Intercept }+X \text { Coefficient } \times x\left(\text { hand }_{(m)}-\text { shoulder }_{(m)}\right)\right. \\
& \left.+Y \text { Coefficient } \times y\left(\text { hand }_{(m)}-\text { shoulder }_{(m)}\right)\right) \times 100 \%
\end{aligned}
$$

Where: $\mathrm{x}\left(\right.$ hand $_{(\mathrm{m})}$-shoulder $\left.(\mathrm{m})\right)$ is the forward/backward hand location with respect to the shoulder joint and $y\left(\right.$ hand $_{(\mathrm{m})}$-shoulder $\left.(\mathrm{m})\right)$ is the hand location distance from the shoulder in the up/down direction.

Table 2 .Equation coefficients and $\mathrm{R}^{2}$ values for the shoulder models used in the tool, where, $\mathrm{X}$ and $\mathrm{Y}$ Coefficients are for horizontal and vertical components of the equation, respectively.

\begin{tabular}{cccccc}
\hline & Data Set & $\mathrm{R}^{2}$ & Intercept & X Coefficient & Y Coefficient \\
\hline & 5\%ile Female & 0.86 & 0.626 & 7.878 & 2.360 \\
& 50\%ile Female & 0.86 & 0.862 & 9.977 & 2.929 \\
Net Shoulder & 95\%ile Female & 0.86 & 1.246 & 13.721 & 3.841 \\
Moment (Nm) & 5\%ile Male & 0.87 & 0.816 & 10.221 & 2.806 \\
& 50\%ile Male & 0.87 & 1.169 & 13.596 & 3.756 \\
& 95\%ile Male & 0.88 & 1.713 & 17.960 & 4.985 \\
\hline & 5\%ile Female & 0.82 & 0.020 & 0.266 & 0.099 \\
Net \%MVC & 50\%ile Female & 0.82 & 0.027 & 0.337 & 0.123 \\
(\%) & 95\%ile Female & 0.81 & 0.041 & 0.458 & 0.164 \\
& 5\%ile Male & 0.81 & 0.013 & 0.166 & 0.063 \\
& 50\%ile Male & 0.82 & 0.019 & 0.220 & 0.084 \\
& 95\%ile Male & 0.82 & 0.028 & 0.290 & 0.112 \\
\hline
\end{tabular}

\subsection{Translation of tool input values for shoulder model input}

Hand location coordinates, as inputs to the tool, are measured with respect to an origin on the workstation surface. The shoulder load models, however, require input values of the hand location relative to the shoulder joint. Hand location inputs are made relative to the shoulder joint based on the assumption of a standard sitting distance from the work surface to the body, which is dependent on the choice of a gender and population anthropometry percentile. It is possible that different seated postures and orientations to the workstation will be chosen by the worker. Even with this potential variability, it is likely that over an extended period of time the 
seated positions will average to what has been assumed as the standard seated position in the tool. Subsequently, the gender and population percentile choice allows available anthropometric data (U.S. based - Humanscale - Diffrient et al., 1973) to be applied to make the appropriate adjustments of the inputs. The method used to make this adjustment is summarised in Table 3. Any differences between the anthropometries of the Humanscale data set and those within 4D WATBAK have been deemed to have no significant impact.

It is possible for other anthropometric data sets to be used. Compared to 2003-2006 United States anthropometric data (McDowell et al., 2008), the Humanscale data used was within 1-2 $\mathrm{cm}$ height, with the exception of a $6.4 \mathrm{~cm}$ difference for the $5^{\text {th }}$ percentile, and $4.2 \mathrm{~kg}$ to $32.2 \mathrm{~kg}$ weight. Similarly, for United States data in ISO/TR 750-2:2010(E) (International Organization for Standardization, 2010), the height and weight differences were $0.1 \mathrm{~cm}$ to $6.6 \mathrm{~cm}$ and $2 \mathrm{~kg}$ to $21 \mathrm{~kg}$ for the same percentiles. The weight differences have an impact of 0.1 to $1.7 \mathrm{~kg}$ on total arm weight, should weight be distributed similarly through the different populations, which would have low impact on the shoulder load model in some instances. Differences between anthropometric sets may be considered irrelevant in the intended design application of the tool since the comparison of new design improvements to an old design (an A-B comparison) will not be greatly affected by changes in anthropometrics.

Table 3. Translations used to make task hand location values relative to a shoulder joint location.

\begin{tabular}{l|l|l}
\hline \multicolumn{1}{c|}{ Direction } & \multicolumn{1}{|c}{ Anthropometric Correction } & \multicolumn{1}{c}{ Effect } \\
\hline $\begin{array}{l}\text { Forward-Backward } \\
\text { (FB) }\end{array}$ & $\begin{array}{l}\text { Average sitting distance from the } \\
\text { edge of the workstation }\end{array}$ & $\begin{array}{l}\text { Translates workstation FB distance } \\
\text { to the centre of the body and } \\
\text { shoulder pivot location }\end{array}$ \\
\hline Up-Down (UD) & $\begin{array}{l}\text { Work table height sitting \& shoulder } \\
\text { pivot height sitting }\end{array}$ & $\begin{array}{l}\text { Translates workstation UD distance } \\
\text { to body shoulder pivot height }\end{array}$ \\
\hline Right-Left (RL) & Shoulder pivot width & $\begin{array}{l}\text { Translates workstation RL distance } \\
\text { from the body midline laterally to } \\
\text { the shoulder pivot }\end{array}$ \\
\hline
\end{tabular}

The shoulder models developed from the two dimensional digital human model data require only one horizontal and one vertical input. The vertical component, consistent between the tool inputs and the shoulder model inputs, does not require any adjustment. With only one horizontal input to a shoulder load model, the two horizontal plane values are combined using Pythagorean 
addition to create a single value. The resulting value is used for the horizontal component input to the two dimensional shoulder load model in the tool. Due to the combination of values, any outputs from these shoulder models are therefore considered to be net shoulder moment or net \%MVC.

\subsubsection{3. $\quad$ Net and Cumulative Shoulder Load Outputs}

The tool shoulder models create outputs of net shoulder moment and net \%MVC for the left and right shoulder of each work element listed. This output information is displayed in a table and can be summarised as a graph of task elements versus load magnitude. Cumulative net shoulder moment is determined by multiplying the net shoulder moment for each element by the time for that element and summing all elements in the work cycle. The contribution of reach or move elements to the cumulative net shoulder moment is calculated from the net shoulder moment at the midpoint between successive hand locations multiplied by the movement time between the hand locations.

The combination of the horizontal plane information to generate proper inputs for the two dimensional shoulder load models is the most significant assumption of the shoulder load models used. A two dimensional shoulder load model was chosen for two reasons: (1) a simple shoulder load model was able to be developed from a two dimensional digital human model data set; and (2) the tool development presented in this chapter is a proof of concept of the tool as a whole. More complex, three dimensional models are possible, but would generate three shoulder load outputs. The three outputs would require more interpretation of their meaning and are less likely to be relevant to non-ergonomists, meaning a decrease in the usability of the tool. The concept of monitoring shoulder risk through a single plane of information is not new. Significant work has been done using inclinometers to correlate posture with risk (Hansson et al., 2006; Hansson et al., 2010).

\subsection{Case example of application of the tool}

\subsubsection{Aim}

The case example, using an observation based assessment of a workstation re-design from the previous study of Neumann, et al. (2002), demonstrates the application of the tool to complete an assessment of a light assembly workstation from leading design parameters, rather than the observational approach that was used in the earlier study. The use of the tool will illustrate how 
the impact of design decisions on work cycle and shift demand requirements can be assessed during the design stage.

\subsubsection{Methods}

Workstation layouts to be assessed with the tool were recreated from a previous study completed by Neumann, et al. (2002), who studied the layout changes observationally in a case study of Swedish electronics assembly system. The 'New' (New Design) and 'Original' (Original Design) workstation layouts assessed within the referent Neumann, et al. (2002) study are shown in Figure 9. To demonstrate a design stage evaluation, stock locations and reach requirements for an operator were estimated from pictures and the original information used in the observation based study. Estimated three dimensional values were verified by one of the Neumann, et al. (2002) authors.
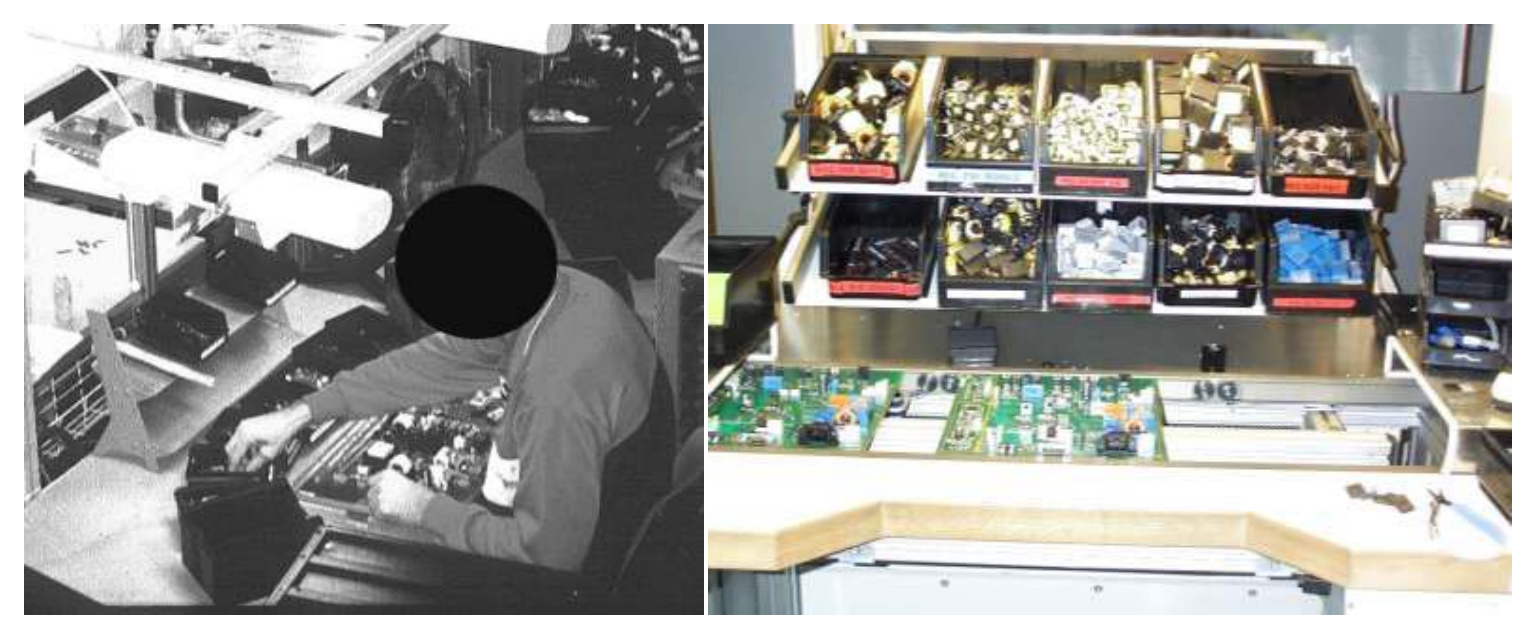

Figure 9. Workstation set up for the original (left) and new (right) workstations from the system studied by Neumann et al. (2002).

All tasks were assumed to be completed with the right hand, as was done in the original observation based Swedish case study. Work tasks progressed sequentially from left to right, top to bottom in each workstation. Stock retrieval was a singular pick and place, meaning two different parts were not grabbed in one reach. A $95^{\text {th }}$ percentile female was used for the shoulder load analysis to match the population assumptions of Neumann, et al. (2002). To assess the design effects over a shift, a full shift was assumed to be 7.5 working hours. The percent change between the New Design and Original Design was calculated from the following equation: 


\section{Workstation Change (\%) \\ $=100 \times($ New Design - Original Design $) /$ Original Design}

\subsubsection{Results}

The observational method (Neumann, et al., 2002) and the virtual method of the tool showed similar trends in the comparison of the two workstations. Both analyses determined that the New Design had increased peak and average shoulder loading for the assembly parts of the work cycle elements. Table 4 highlights the findings from the virtual tool. It shows that the peak Net Shoulder Moment increased by $22.7 \%$ in the change to the New Design. The average Net Shoulder Moment also increased from 7.2 Nm to 7.9 Nm, due in part to the New Design having six elements that exceeded the peak moment of the Original Design. These values corresponded to a $26.4 \%$ and $11.7 \%$ increase in peak and average Net \%MVC, respectively. Further, the virtual method identified a per cycle decrease in the amount of hand travel required and cycle time. When scaled to a full shift, the difference is switched, and the New Design has a 3.7\% increase in cumulative hand movement because of the total work time differences.

Table 4. Comparison between assembly task shoulder load, cycle time and cumulative hand travel calculated values for the proposed virtual tool and the observational method used by Neumann et al. (2002).

\begin{tabular}{|c|c|c|c|c|c|}
\hline & & & Worksta & Design & Workstation \\
\hline & & & Original & New & Change (\%) \\
\hline & Net Shoulder Moment (Nm) & Peak & 9.7 & 11.9 & 22.7 \\
\hline & 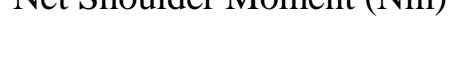 & Average & 7.2 & 7.9 & 9.7 \\
\hline Assembly & Cumulative Net Shoulder M & nt $(\mathrm{Nms})$ & 424.5 & 427.9 & 0.8 \\
\hline & Not 0 MMVC $(0 /)$ & Peak & 31.4 & 39.7 & 26.4 \\
\hline & ivet ronive (\%) & Average & 23.0 & 25.7 & 11.7 \\
\hline Work Cycle & Time (s) & & 64.3 & 58.4 & -9.2 \\
\hline Hand & & & & & \\
\hline Travel & Per Work Cycle (m) & & 11.8 & 10.5 & -10.7 \\
\hline & Per Shift (m) & & 2252.0 & 2336.0 & 3.7 \\
\hline
\end{tabular}

The virtual tool quickly allows hand movement for the two setups to be displayed. Hand locations relative to identified acceptable frequent (inner or lower dashed line) and occasional 
(outer or upper solid line) work zones are shown for the above view (horizontal plane), in Figure 10, and side view (vertical plane) in Figure 11. From the overhead view, the Original Design has a work envelope that is closer to the worker than with the New Design for most elements. The side view shows that all original design tasks are within the frequent vertical reach zone while the New Design has tasks in multiple zones. The side view also supports the observation of the distance of reach required that was evident in the overhead view.

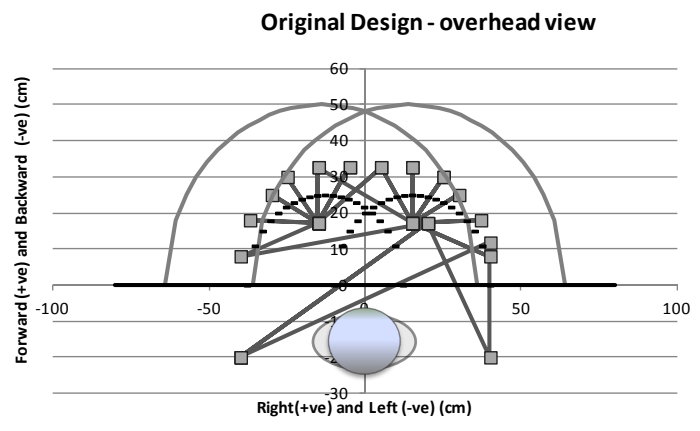

(A)
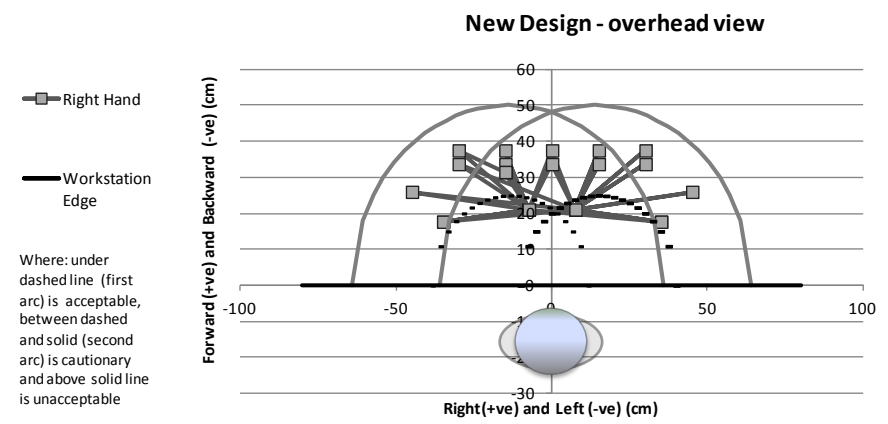

(B)

Figure 10. Overhead view (of the hand locations, relative to the workstation, required to complete the work elements for the Original Design (A) and New Design (B).

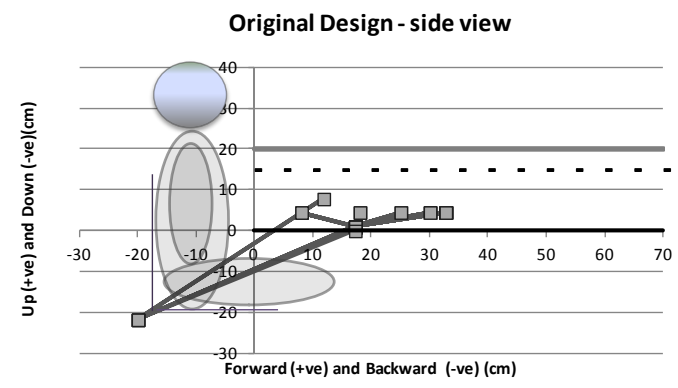

(A)

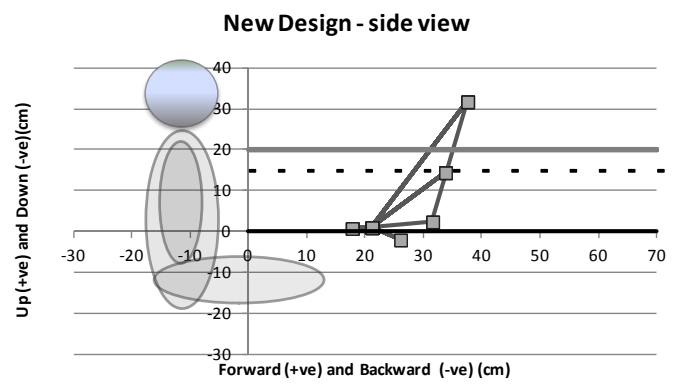

(B)

Figure 11. Side view of the hand locations, relative to the workstation, required to complete the work elements for the Original Design (A) and New Design (B).

The hand travel distances between elements and cumulative distance are shown for the virtual tool in Figure 12. The movements required at the beginning and end of the work cycle of the Original Design can be seen as key contributors to the hand travel differences between the two 
designs. These correspond to the points away from the main cluster of points in Figure 10 (A) and Figure $11(\mathrm{~A})$.
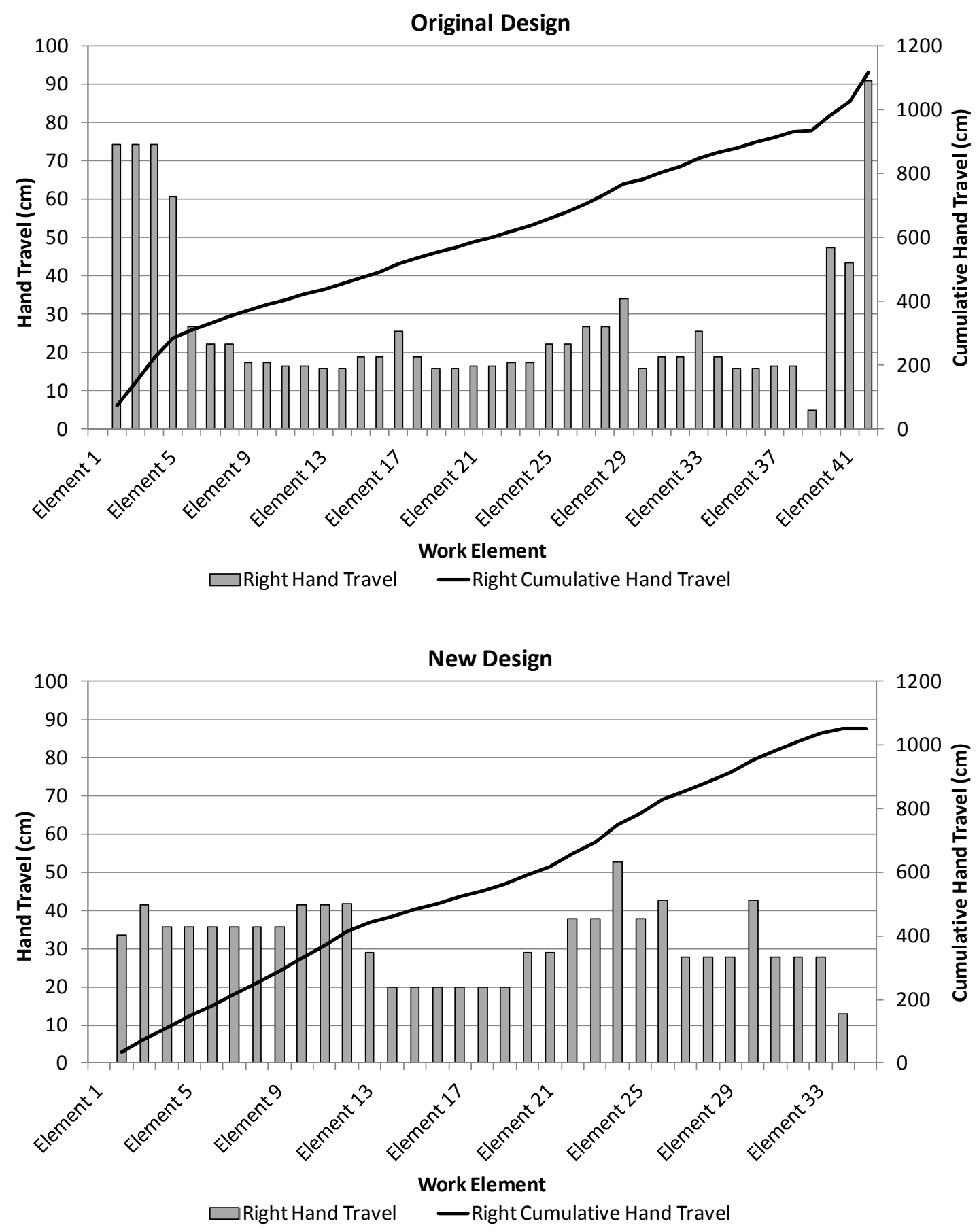

Figure 12. Hand travel between elements and cumulative hand travel for the original workstation (top) and new workstation (bottom). (Note: Element labels changed for simplicity of presentation). 


\subsection{Discussion}

This chapter presents the concept of a light assembly workstation design assessment tool and a case example demonstrating its benefit in making layout decisions. The tool converts available workstation information (hand location and work element descriptions) into outputs that report on system (time) and human (work element and cumulative hand travel and shoulder loading) outcomes for practitioners. The current simple shoulder load models are openly shared here to allow further development in other applications. More complex biomechanical models could be incorporated in future versions - provided they retain the ease of use embedded in this tool which requires only layout coordinates and no special training to use. In the tool's current form, as guided by industry feedback (Greig et al., 2013b), it provides a reliable method to quantify and compare design decisions, in both ergonomic and performance terms that are easily used by non-ergonomics specialists with limited training in predetermined motion time systems. Such simplicity in an integrated perspective is currently not available from other light assembly design tools. The tool does not replace all HF analysis tools but instead should be considered as part of a suite of risk analysis tools. Ideally the tool will be used to better understand workstation layout and work assignment choices allowing for both improved performance and reduced injury risks for operators.

The case in this chapter demonstrates the ability and potential benefits of this novel, virtual tool. Using workstation layouts, this tool predicted worker load levels that were similarly identified in an observation based workstation assessment by Neumann, et al. (2002). The virtual tool identified the same change trends between the two workstation designs even with subtle differences of estimated hand locations and task elements from the actual workstations. Further, the virtual tool provides a greater range of risk analysis outputs by combining hand travel, reach zone acceptability, task element time prediction, and an estimation of shoulder loading for the task elements and work cycle. Together this information allows change proposals to be assessed and targeted workstation improvements to be completed as required before any change is implemented. 
The ability for design stage assessment is a primary benefit of the tool over the observation method of Neumann, et al. (2002). Design decisions can then be connected to worker load and task element time at a stage where the ability to make change is more feasible (Miles and Swift, 1998; Dul and Neumann, 2009). A secondary benefit is the ability to bring together a range of outputs to obtain a better appreciation of the simultaneous impact of the layout choices on the worker and the work system.

The New Design was proposed to improve productivity, meaning reduce cycle time through the addition of a transport conveyor. Adding the conveyor reduced the hand movement when transitioning to the next cycle, a contributor to the reduced work cycle cumulative hand travel in the New Design. The addition of more HF-related information in the assessment from the tool begins to show that the benefit of the change actually increases risk for the worker. The peak net shoulder moment output was not only elevated in the New Design, as was found by Neumann, et al. (2002), but the peak net shoulder moment from the Original Design was actually exceeded by six tasks in the New Design. Similarly, reach locations, as summarized in the reach zone graphs, showed how the conveyor moved the work further from the worker and induced higher reaches, some of which would be above the shoulder level of a $5^{\text {th }}$ percentile female. Scaling the tool outputs to reflect activity for a full shift, the hand travel, which had decreased within a cycle and reduced cycle time, now showed an increased cumulative hand travel of $3.7 \%$ over the Original Design for a full shift. Likewise, the cumulative shoulder load showed a slight increase in a work cycle of the New Design (0.8\%), meaning a greater load over the course of a shift. Had a design engineer looked at the tasks and work cycle in isolation, the work shift information would have likely been overlooked. However, the cumulative effect of the work cycle tasks has been presented in an easily scalable way to look at the combined effect of all tasks throughout a working shift. Workstation layout changes can be investigated with greater effectiveness in this way with this tool, and far earlier in the change process than other HF tools allow.

The information from the tool could, conceivably, be applied to line balancing decisions, similar to Carnahan, et al. (2001), as well as to help create an optimal layout and work distribution, as has been proposed by Braun, et al. (1996) and Ben-Gal and Bukchin (2002), or used to make more informed decisions earlier in design proposals as was demonstrated in the case example. 
When applied early in workstation design the tool easily depicts the worker and workstation interaction, which can help with the interpretation of design concepts. Similar to how Braun, et al. (1996) and Zha (2001) used limb anthropometrics to anticipate worker reach envelopes and guide stock placement, the tool uses industry accepted horizontal and vertical reach zones which give a simple illustration of how the different task locations align with the $5^{\text {th }}$ percentile worker. Coupling the hand location output information with the corresponding shoulder load means that frequently completed tasks, or those with heavier loads, are more easily identified and able to be prioritised for improvement or preferred workstation locations that reduce the worker burden. This virtual tool analysis is not just limited to early design but can be completed at any stage of the design process. Hand location values can be readily obtained from digital design drawings, pilot build mock-ups or when the final system is completed. A revision of hand locations in any of these stages causes all outputs to change accordingly, which can help to guide the design process. This type of combined information analysis is possible using the observation based approach of Neumann, et al. (2002), but requires the physical station to be in place with a worker present and separate analysis processes to obtain the necessary information contained in this tool. Though workstation assessments and changes post-design are an accepted practice, assessments and changes that are able to be completed in the design stage have been shown to be more easily implemented, more effective, and have greater impact because of the avoidance of costly retrofitting (Miles and Swift, 1998; Neumann and Dul, 2010).

Performance time and load time estimations in the tool helps to connect HF information to work system components commonly of interest to system designers, and allows HF practitioners to capture the impact of cumulative work. With the connection to the work system, system designer goals to reduce cycle time can be better matched with HF practitioner desires to reduce reach distance and shoulder load, which creates cross discipline understanding and benefits to the worker well-being and system performance objectives that are at the core of HF (Dul, et al., 2012). Capturing cumulative work exposure allows a more realistic picture of task element shoulder demands on the worker within the cycle, and with simple scaling, for the work shift. Cumulative loading is a measure likely more reflective of total worker demand. It has been shown to be an important risk factor for low back pain, resulting in the development of exposure thresholds (Norman, et al., 1998). Thresholds for cumulative shoulder loading are being 
developed but have not been established to the same extent as for the low back (McClellan et al., 2009; Seaman et al., 2010).

With system and HF information together in the tool, the more complete picture of work cycle demands could use the time and worker demand information to better assign and distribute work tasks in a workstation (or, potentially, on a whole line) to better balance worker demand. Previous researchers have investigated the connection between the system, HF, and line balancing (for example: Braun, et al., 1996; Carnahan, et al., 2001; Zha, 2001; Ben-Gal and Bukchin, 2002). However, their HF measures do not relate well to light assembly and the complexity of their procedures might inhibit usability by people outside of their domain. Future work to improve the calculation of time-based exposure measures will create a better understanding of the impact of design decisions during the design stage and, through the use of the time element, improve the connection of HF with design engineers (Wells, et al., 2007).

The tool has also been created to address a need to incorporate HF assessment into system design decisions via a simple, yet informative, and cost effective tool. Perez and Neumann (2015) identified nine characteristics that motivate or deter the use of a virtual human factors tool. These characteristics include: time, cost, training, difficulty to use, trustworthiness, graphics, flexibility, usefulness and report presentation. It is believed that this tool satisfies a number of these factors, specifically:

1) Inputs to the tool have been designed to be as simple as possible: distances between points on a CAD drawing, or use of a tape measure, in the case of measurements on an existing workstation.

2) The Excel-based format has been used to allow the tool to be readily accessible and cost effective compared to other predictive tools like Jack, Santos, or even 4D WATBAK.

3) Results interpretation is straightforward, even for non-experts (Greig, et al., 2013b), and clearly presented for system and human outcomes.

Time to use the tool, a key factor outlined by Perez and Neumann (2015), presents the greatest challenge to the tool in its current form. Describing all of the work elements, necessary for MTM-1 and calculation of some tool outputs, can be an arduous task and requires specific training. MTM is used in industry and has various forms possible to describe tasks (e.g. MTM- 
UAS, MTM-MEK). It is not always applied though at the detailed level of MTM-1, in part due to the time consuming nature of describing work at the element-by-element level of detail.

With time prediction in the tool, the length of exertion time can be estimated. Exertion time, coupled with exertion intensity (\%MVC), can be used as inputs to maximal endurance time prediction models (for example, Mathiassen and Åhsberg, 1999; El ahrache et al., 2006). Corresponding pause or recovery times, from rest allowance models (for example, Rose et al., 1992; El ahrache and Imbeau, 2009), could inform a user on appropriate work-to-rest ratios. This functionality, applied similarly by Carnahan, et al. (2001) and Dode (2012), is currently under development for future versions of the tool.

Tool validation and epidemiologic calibration of the outcomes are also points of future work. Error sensitivity testing that has been completed has found that hand loads up to $100 \mathrm{~g}$ differed from the tool's base model predicted shoulder load by 5\%-12\%; hand location measurement error averaged within $1 \mathrm{~cm}$ of referent values; while simulated input error resulted in an average of $<0.05$ Nm predicted shoulder load and < $3.6 \mathrm{~ms}$ predicted movement time (Greig and Neumann, 2015). These testing results demonstrate the acceptability of the tool in a light assembly application such as electronics assembly. They also suggest that possible inertial limb-load effects of dynamic movements would have little impact on the results and be negligible in the comparison of two design options (e.g. A to B comparison).

The case application presented within this chapter was used to illustrate the tool and help prove the concept. The comparison of outcomes to a real, observed case does provide an initial, basic examination of content validity. More work is required to systematically assess the validity of the tool content and other aspects of validity, such as construct and face validity, as well. Calibration of output measures to known thresholds (for example see ACGIH HAL-TLV (American Congress of Governmental Industrial Hygienists, 2001); NIOSH Action Limit (National Institute for Occupational Safety and Health, 1994)) would also provide useful extensions of the current tool. With appropriate thresholds and limit values for both the work cycle and the shift, users of the tool will have more defined targets to work towards to maximize worker performance. Thresholds have been shown to be useful in assessing conformance to 
standards and can also provide a simple statement on usability (Lee and Koubek, 2012). Both of these applications could be beneficial in workstation design. Currently, shoulder load thresholds remain a work in progress (McClellan, et al., 2009; Seaman, et al., 2010) and the concept of hand travel is even less developed.

\subsection{Conclusion}

A novel tool is presented that predicts worker demand and element time in light assembly from workstation layout and task elements using simple biomechanical regression models and Methods-Time Measurement. Right and left hand element description inputs are used to generate estimations of hand travel, zones of reach acceptability, and shoulder load for male and female $5^{\text {th }}, 50^{\text {th }}$, and $95^{\text {th }}$ percentile populations. The tool can be used at any time during the design stage to assist in line layout, workstation layout, and task balancing. Work station design induced worker demands, assessed in a case example using this tool, compared favourably to an established observation based method. The virtual method can be considered an effective replacement for the observation based method in the context examined. This new tool has the ability to provide a greater breadth of assessment information that can allow workstation and system designers to develop and review workstation proposals at the earliest stages of workstation design. 


\section{Chapter 5}

\section{Testing of a workstation efficiency evaluator tool}

\section{Introduction}

This chapter addresses dissertation research questions 2.2 to 2.5 in pursuit of objective 2:

Develop a workstation level HF assessment tool for light assembly work. The specific research questions are as follows:

Research Question 2.2 - How do tool shoulder load moment outputs for different hand loads differ from the shoulder load moment outputs of the tool baseline models?

Research Question 2.3a - What is the average magnitude of error in hand location measurement?

Research Question 2.3b - Does the magnitude and direction of input error differ between measures taken from a virtual workstation and a matching physical workstation? (RQ 2.3a)

Research Question 2.4 - How does the magnitude and direction of induced hand location input error affect a) predicted shoulder load outputs and b) estimated reach/move time outputs of the tool?

Research Question 2.5 - Using the results on the characteristics of hand location input error (RQ 2.3a, 2.3b) and the outcomes from testing induced measurement error (RQ 2.4), how large is the potential error due to the observed input measurement variability?

This chapter extends on the tool developed in the Chapter 4. The focus in this chapter is on determining response of the tool to input errors and hand loads that differ from the assumed hand load in the tool.

\subsection{Aim}

The purpose of this chapter is to examine the WEE tool developed by Greig, et al. (2013a) in order to understand the impact of different hand loads on shoulder load outputs, the 
characteristics of hand location measurement error and the impact of hand location input error on the time and shoulder load outputs of the tool. This will be assessed through three studies guided by specific research questions (RQs).

In the first study, to understand the tool's current assumption of zero hand load, we ask:

- How do tool shoulder load moment outputs for different hand loads differ from the shoulder load moment outputs of the Greig, et al. (2013a) tool baseline models? (RQ 1.0) The second study, that helps to understand the errors from potential users, is guided by RQs that ask:

- What is the average magnitude of error in hand location measurement? (RQ 2.0)

- Does the magnitude and direction of input error differ between measures taken from a virtual workstation and a matching physical workstation? (RQ 2.1)

Study three examines the impact of simulated hand location input error on tool outputs and combines the findings of study two to determine the impact of user inputs. This study is guided by the following RQs:

- How does the magnitude and direction of induced hand location input error affect a) predicted shoulder load outputs and b) estimated reach/move time outputs of the tool? (RQ 3.0)

- Using the results on the characteristics of hand location input error (RQ 2.0, 2.1) and the outcomes from testing induced measurement error (RQ 3.0), we ask: how large is the potential error due to the observed input measurement variability? (RQ 3.1)

\subsection{Methods}

Three studies were completed to address each of the three core RQs identified earlier. We begin by describing the Workstation Efficiency Evaluator (WEE) tool being tested.

The WEE tool developed by Greig, et al. (2013a) has inputs of hand location (cm) and MTM-1 description (cf. Freivalds and Niebel, 2009) for each element along with a global, non-element specific classification of population percentile to be examined. Tool inputs are processed to be used in developed shoulder load models and for time estimation, the focus of this chapter. Other outputs of the tool include hand travel distance and identification of acceptable reach zones. The Greig, et al. (2013a) zero hand load shoulder load models, meaning no hand load is assumed and no hand load input is required in the model, were created from linear regression of data sets 
derived from a two dimensional digital human model containing horizontal and vertical hand location and associated shoulder load. Shoulder moment and percent capability (\%MVC) models were created for male and female populations $\left(5^{\text {th }}, 50^{\text {th }}, 95^{\text {th }}\right)$. The two dimensional shoulder load models give a single moment or \%MVC output that are considered net shoulder moment and net \%MVC because of the combination of the horizontal plane hand location input measures to create two distance inputs for the models. The zero hand load models matched the initial application of the tool with light assembly (e.g. electronics) where parts and devices are quite light and a number of the hand movements, up to half, can be completed without any external hand load. The MTM-1 element descriptions are used to generate process time (s), the time needed for getting and placing, or movement time (s), the time needed to reach with an empty hand to obtain something (reach time) or move something to a new location (move time).

\subsubsection{Study 1: RQ 1.0 - hand load effects}

The variation in predicted shoulder load due to increasing hand loads was examined by creating eight new sets of predictive shoulder load models. All shoulder load models were compared using a standard hand reach location test data set that was used to generate shoulder load outputs from all models.

\subsubsection{Model development}

The new sets of shoulder load prediction models (25, 50, 100, 200, 400, 700, $1000 \mathrm{~g}$ and for hand load as an input variable) were developed for male and female $5^{\text {th }}$, $50^{\text {th }}$ and $95^{\text {th }}$ percentile of populations that included hand load using the technique originally presented by (Greig, et al., 2013a). Briefly, a two dimensional digital human model (4D WATBAK, Version 2.0.37 Ergowatch, University of Waterloo, Canada; Neumann, et al., 1999) was systematically positioned with a range of arm locations at $30^{\circ}$ intervals, starting with the arm vertically downward at the side, up to a $90^{\circ}$ shoulder flexion angle. For each shoulder angle the elbow was systematically varied (flexed) by $30^{\circ}$ through a set of angles $\left(0^{\circ}-120^{\circ}\right)$ as well. Zero hand load was previously used for these positions creating the base model of the tool. The seven load levels outlined above were added for this study. The vertical and horizontal hand location relative to the shoulder was recorded for each of the 19 limb positions along with the corresponding shoulder moment. A second shoulder load representative value was also calculated from the shoulder moment as a percentage of the average population maximum shoulder moment capability as specified by the software (cf. Neumann, et al., 2002). Linear 
regression was applied to the data sets to generate two models, one for shoulder moment (Nm) and one for percentage of maximum voluntarily capable (\%MVC), each as a function of the horizontal and vertical hand location relative to the shoulder.

The new sets of models differed from the original zero hand load models in that seven of the models had a specific hand load, noted above, for the digital human model that was used to generate the data set, termed 'specific load models' (SLM). The one remaining set of models, termed 'general load models' (GLM) used hand load magnitude as an additional input to the model. The data set for the GLM models combined the data from the original base model and the seven developed load specific models to generate the shoulder moment and \%MVC models for this last set. Linear regression of the GLM data sets created shoulder load prediction models that were a function of the horizontal and vertical hand location and hand load.

\subsubsection{2. $\quad$ Data set to test the models}

An input test data set containing 84 hand locations was created to compare the impact of hand load on the shoulder load outputs for each model. Twenty-one points representing hypothetical reach locations were spread over a representative workstation as shown in Figure 13 and repeated in four vertical levels $0,10,20$, and $30 \mathrm{~cm}$ above the workstation work surface.

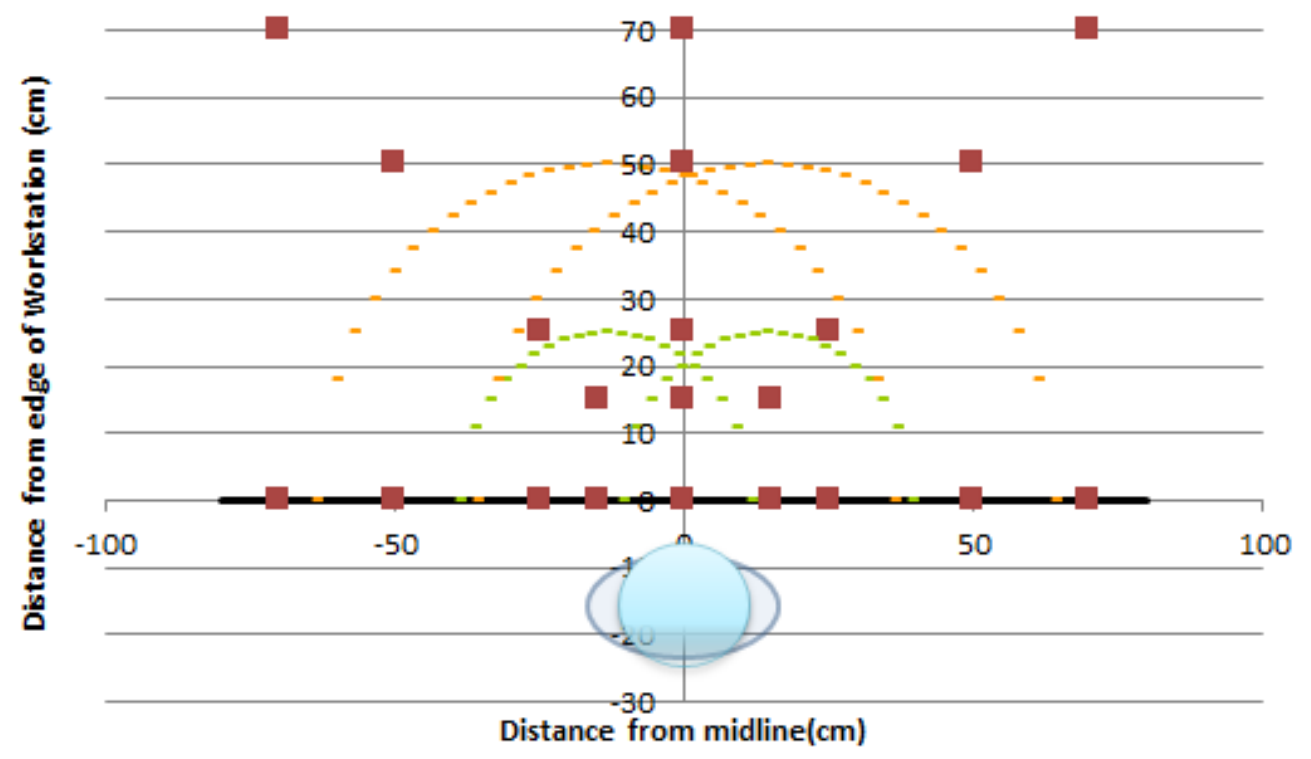

Figure 13. Data points within the test data set for the horizontal plane. All points were repeated for 0, 10, $20,30 \mathrm{~cm}$ above the workstation work surface $(\mathrm{n}=84)$. 


\subsubsection{3. $\quad$ Data testing and analysis}

The impact on the shoulder moment and \%MVC for each hand location was determined by comparing the outputs for each model with the referent output values of the base models used in the tool. Descriptive statistics were used to summarize the relationship between the different models and the base model.

\subsubsection{Study 2: RQ 2.0 - hand location measurement error characteristics}

To understand the characteristics (magnitude and direction) of human errors in measuring hand location, a lab based study was completed in which participants completed a series of workstation hand location measurements on virtual (CAD drawn) and physically constructed workstations. Twelve participants from a Mechanical and Industrial Engineering undergraduate program were recruited as volunteers to complete the study. All participants were required to have experience using SolidWorks (Dassault Systèmes Corporation) software. Informed consent was obtained from all participants prior to their participation.

\subsubsection{Setup}

Five different workstation layouts were used in this study; all were plausible layouts of a light assembly workstation (Figure 14). Each workstation had five hand locations to be measured. Four virtual workstations were presented to participants using SolidWorks. The other workstation was physically constructed in the lab (see Figure 14). One of the four virtual workstations had the identical layout of the physical workstation. 


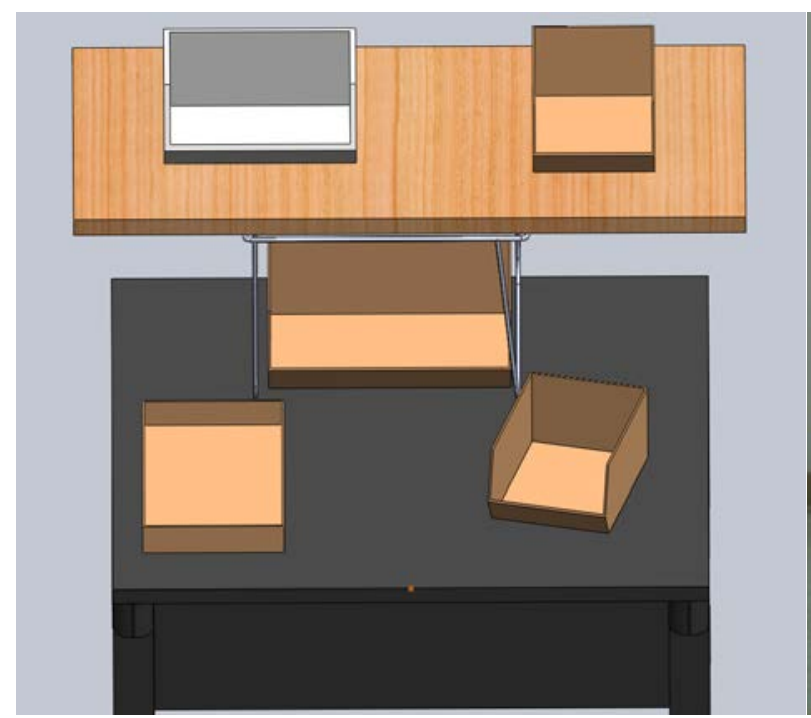

a)

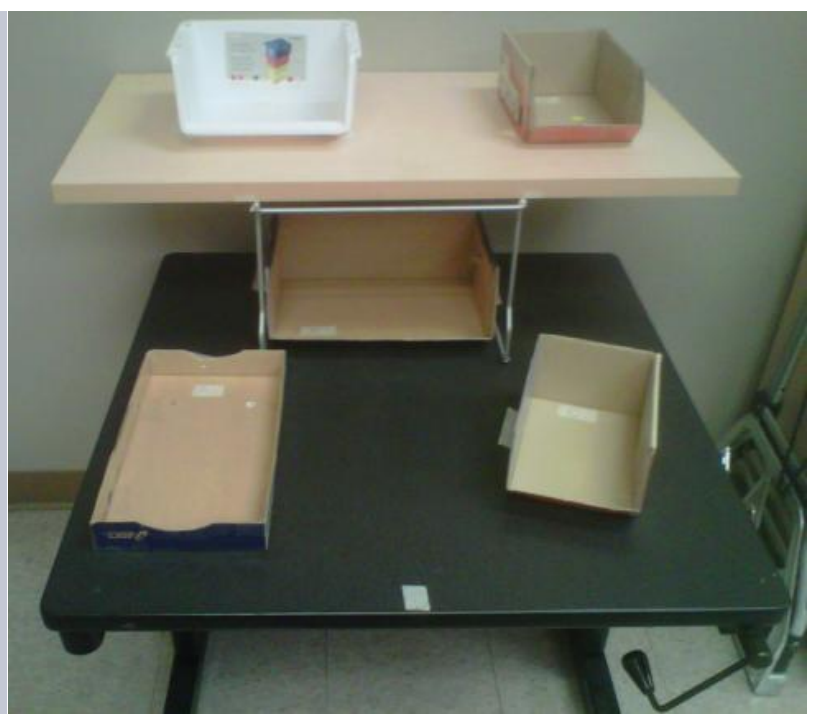

b)

Figure 14. Examples of a) one of the virtual workstation and b) the matching physical workstation used for data collection.

\subsubsection{Data collection}

Participants measured the three dimensional hand locations (FB-forward/backward, RLright/left, UD-up/down) from an origin on the top, front, middle of the workstation to a point representing the average hand location for continuous work from a given bin location - the centroid of the bin. Measurements within SolidWorks were completed using a measuring tool within the program. A tape measure was used to measure location distances on the physical workstation.

The presentation of the physical workstation was balanced so that half of the participants completed its measurements at the start and the other half completed it at the end of the trials. The presentation of the four virtual workstations was randomized within its block either before or after the physical workstation. The virtual workstation sequence was also balanced so that each workstation appeared an equal number of times in each sequence spot.

\subsubsection{Data Analysis}

The location measured for each point was compared to the precisely measured, desired location established by the researchers - deemed the referent. Distances from the referent, defined as measurement error, were calculated. The average, minimum, maximum and standard deviation of error were calculated within the RL, FB, UD directions. The difference between 
measurements from a virtual workstation and a physical workstation was determined by comparing the average measurement error taken with the tape measure to those from the measuring tool in its virtual counterpart for each of the three measurement directions.

\subsubsection{Study 3: RQ 3.0 - Hand location input error effect on load and time outputs}

To understand the impact of hand location input errors on tool outputs for shoulder load and movement time, the 84 hand location reference test data set in Study 2 was entered into the tool with the corresponding outputs noted. Each reference location was then systematically adjusted in all axes and for all combination between axes by a magnitude of 1, 2, 5, or $10 \mathrm{~cm}$. Resulting shoulder load (Nm and \%MVC) and movement (reach and move) time outputs were recorded for each point. This process was completed for the male and female $5^{\text {th }}, 50^{\text {th }}$ and $95^{\text {th }}$ percentile populations. To create a consistent comparison across movement time data, reach and move time for each hand location was determined to and from a common, fixed point on the workstation.

Average differences from the reference location outputs were calculated for the 1, 2, 5, and 10 $\mathrm{cm}$ adjustment level. Similarly, the absolute difference from the reference was also determined. Descriptive statistics of the difference from the reference point were used to summarize the findings.

Results of Study 2 (RQ 2.0) were subsequently compared to the results of this study to determine the magnitude of the potential output error due to average input variability of the participants. Separate regression equations were created for shoulder load moment and move time for each of the RL, FB, UD directions based on the magnitude of induced error and the average output error response. Equations were generated in Microsoft Excel and were chosen based on the higher magnitude of $\mathrm{R}^{2}$ for either a linear or second order polynomial trend line. Using the findings from Study 2, the average, average plus one standard deviation, and the average minus one standard deviation for the location measurement error was entered into each equation to determine the average shoulder load moment or move time error due to participant error. As a further examination of the impact of input error on shoulder load and time output, values from 
the average location measurement error of Study 2 were added to each of the 84 test data points. Descriptive statistics were determined for predicted shoulder load and movement time to examine the difference from the original values of the same test data set.

\subsection{Results}

\subsubsection{Study 1: RQ1.0- hand load effects}

The equation coefficients and $\mathrm{R}^{2}$ values for all models developed are included in Appendix C.

Examples for a $50^{\text {th }}$ percentile male for $25 \mathrm{~g}$ specific load model (SLM) and for the general load model (GLM) are shown below.

\section{5 g SLM:}

Net Shoulder Moment $(\mathrm{Nm})$

$$
\begin{aligned}
& =0.019+0.224 \times x\left(\operatorname{hand}_{(m)}-\operatorname{shoulder}_{(m)}\right)+0.085 \times y\left(\operatorname{hand}_{(m)}\right. \\
& \left.-\operatorname{shoulder}_{(m)}\right)
\end{aligned}
$$

GLM:

$$
\begin{aligned}
& \text { Net Shoulder Moment (Nm) } \\
& =0.027+16.636 \times x\left(\operatorname{hand}_{(m)}-\text { shoulder }_{(m)}\right)+3.734 \\
& \times y\left(\operatorname{hand}_{(m)}-\operatorname{shoulder}_{(m)}\right)+0.004 \times\left({\text { hand } \left.\operatorname{load}_{(g)}\right)}\right.
\end{aligned}
$$

Where: $x$ (hand $_{(m)}$-shoulder $\left.(m)\right)$ is the forward/backward hand location (m) with respect to the shoulder joint, $y\left(\right.$ hand $_{(m)}$-shoulder $\left.(m)\right)$ is the hand location distance (m) from the shoulder in the up/down direction and hand $\operatorname{load}_{(g)}$ is the hand load (g) lifted.

As might be expected, all models predicted a higher level of shoulder load compared to the zero hand load base models used in the tool. The magnitude of difference increased with the size of the hand load. The effects of changing hand load in the shoulder load models for a $50^{\text {th }}$ percentile male are shown in Figure 15, and reflect the general trend of all anthropometrics examined. In the $50^{\text {th }}$ percentile male example, the GLMs exceeded the tool's zero hand load base models by 5\% to 54\% for the range from $0 \mathrm{~g}$ to $1000 \mathrm{~g}$, respectively - equivalent to an average of approximately $0.7 \mathrm{Nm}$ to $4.4 \mathrm{Nm}$ or $1.2 \% \mathrm{MVC}$ to $6.7 \% \mathrm{MVC}$. The SLMs averaged $1 \%$ to $65 \%$ greater than the tool's base models for the hand loads ranging from $25 \mathrm{~g}$ to $1000 \mathrm{~g}$, 
respectively - equivalent to approximately $0.1 \mathrm{Nm}$ to $5.9 \mathrm{Nm}$ or $0.2 \% \mathrm{MVC}$ to $9.4 \% \mathrm{MVC}$, and showed less variability compared to the GLM. Hand load values up to 100 g predicted shoulder load values within $6 \%$ of the tool outputs for SLM and within 10\% for GLM. Across all percentiles, the trends seen in the example were similar though the magnitude of the difference from the zero hand load base model was scaled based on the percentile examined. This means that the average difference from the base measures decreased as anthropometry was changed from the $5^{\text {th }}$ percentile female through to the $95^{\text {th }}$ percentile male. For hand loads up to $100 \mathrm{~g}$ the difference in the SLMs ranged from $12 \%$ ( $5^{\text {th }}$ percentile female) to $5 \%$ (95 ${ }^{\text {th }}$ percentile male) while the GLM was $18 \%$ to $7 \%$, respectively. Similarly, the maximum difference of a single point in the test data set for the $1000 \mathrm{~g}$ SLM ranged from $210 \%-150 \%$, respectively, for the same percentiles. The average difference from the predicted shoulder moment of the zero hand load base model had a range across the percentiles of $0.55 \mathrm{Nm}$ to $0.62 \mathrm{Nm}$ at $100 \mathrm{~g}$ for the SLM and $1.01 \mathrm{Nm}$ to $1.12 \mathrm{Nm}$ for the GLM. At $1000 \mathrm{~g}$ the SLM ranged from an average of $5.6 \mathrm{Nm}$ to 6.1 Nm while the GLM differed by an average of $3.9 \mathrm{Nm}$ to $4.7 \mathrm{Nm}$.

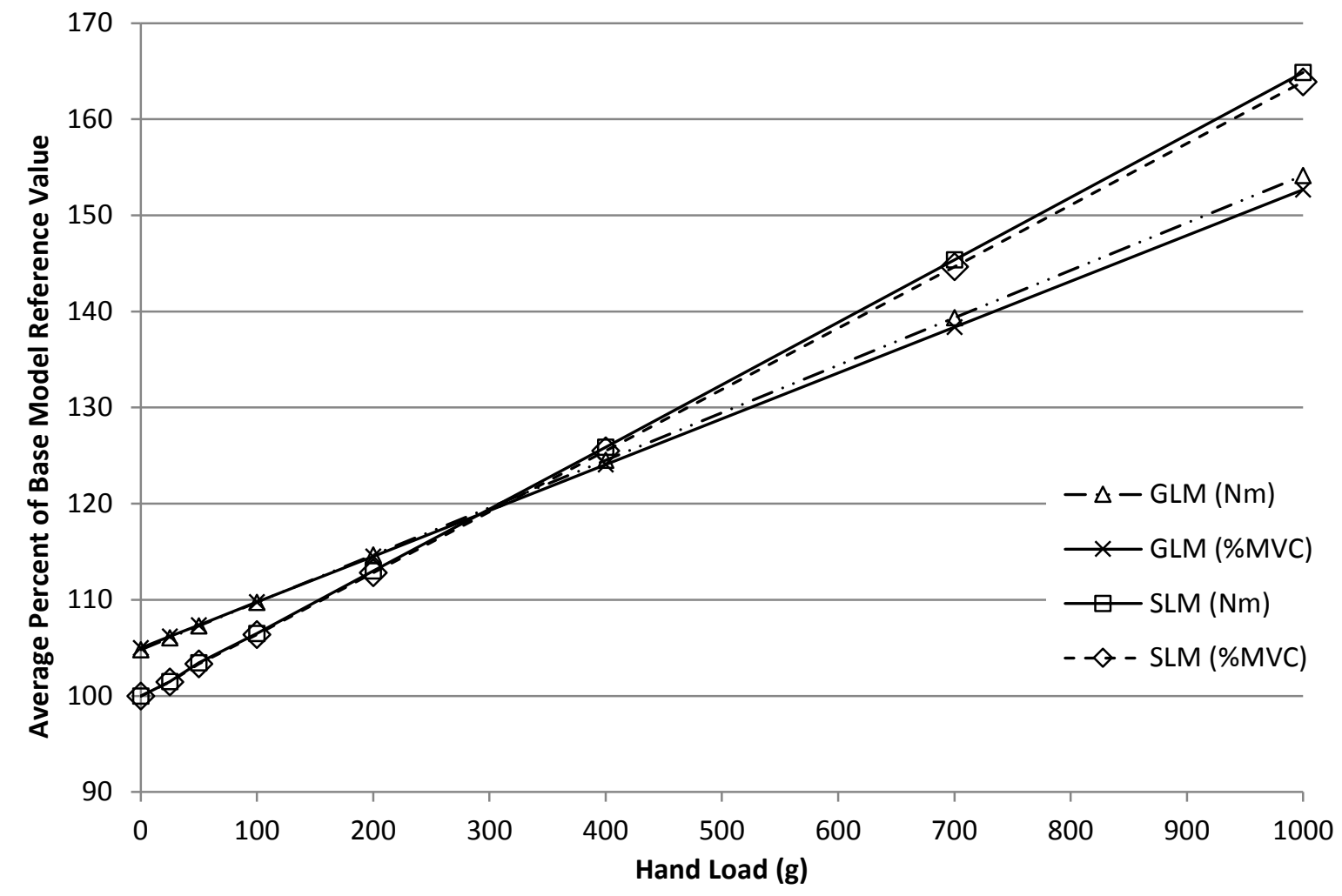

Figure 15. Test measure as a percentage of the reference value for general shoulder load models (GLM) and specific load models (SLM) for a 50th percentile male. 


\subsubsection{Study 2: RQ 2.0 - hand location measurement error characteristics}

The average and standard deviation of measurement errors for the physical and virtual workstations, along with the subset digital workstation matching the physical workstation, are shown in Figure 16. Mean error ranged from $-0.64 \mathrm{~cm}$ to $0.48 \mathrm{~cm}$. The greatest single measurement error was seen in the UD direction $(-11.17 \mathrm{~cm}$ and $10.0 \mathrm{~cm}$, virtual and physical workstations, respectively) whereas the lowest maximum error for a direction was $-2.22 \mathrm{~cm}$ (left direction). Standard deviations of error ranged from $0.56 \mathrm{~cm}$ in the virtual RL to $2.48 \mathrm{~cm}$ in the virtual UD. Physical workstation measurement error was only the highest error in the RL direction when compared to the average error in all of the virtual workstations. When comparing physical workstation measurement error to the virtual workstation measurement error of a matched location, physical workstation measurement error was greater in the UD and RL direction (Figure 16).

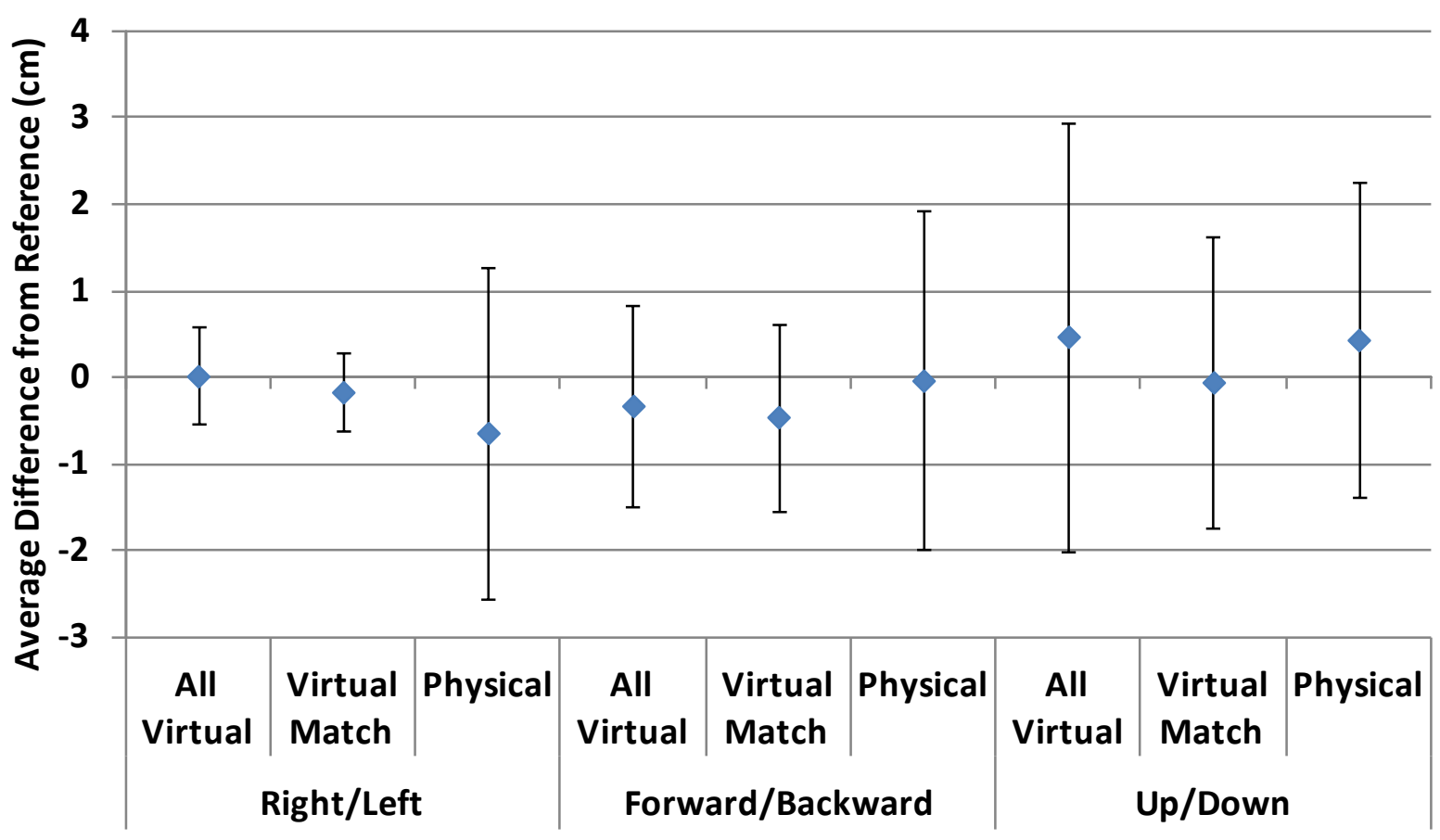

Figure 16. Average error +/- one standard deviation for all axes, where Virtual Match, a subset of All Virtual, is the virtual workstation that matches the physical workstation. 


\subsubsection{Study 3: RQ 3.0 -Hand location input error effect on load and time outputs}

\subsubsection{Shoulder Load}

Representative, average predicted shoulder loads from simulated location input error in the test data set are shown for a $5^{\text {th }}$ percentile female in Table 5 . Over all data points, for each percentile tested, there was an average difference from the original reference value that ranged from 0.02 $\mathrm{Nm}$ to $0.04 \mathrm{Nm}$ and $0.04 \% \mathrm{MVC}$ to $0.11 \% \mathrm{MVC}$. Average error within $2 \mathrm{~cm}$ of the reference data point for all percentiles was $0.003 \mathrm{Nm}$ to $0.005 \mathrm{Nm}$ and $0.05 \% \mathrm{MVC}$ to $0.013 \% \mathrm{MVC}$ from the reference value. Average absolute error ranged from $0.26 \mathrm{Nm}$ to $0.60 \mathrm{Nm}$ and $0.57 \% \mathrm{MVC}$ to $1.55 \% \mathrm{MVC}$ across all populations and points tested. For errors of $2 \mathrm{~cm}$, average absolute error was $0.12 \mathrm{Nm}$ to $0.27 \mathrm{Nm}$ and $0.44 \% \mathrm{MVC}$ to $0.69 \% \mathrm{MVC}$. As an absolute percentage of the reference value, both the average $\mathrm{Nm}$ and $\% \mathrm{MVC}$ were less than $2.5 \%$ of the referent base value for a $2 \mathrm{~cm}$ input error.

Table 5. Representative average response of the shoulder load outputs to simulated input error for a 5th percentile female across the 84 point test data set.

\begin{tabular}{c|ccc|c|ccc|c}
\hline \multirow{2}{*}{$\begin{array}{c}\text { Simulated } \\
\text { Error }\end{array}$} & \multicolumn{3}{|c|}{ Shoulder Moment Error (Nm) } & \multicolumn{3}{c}{ Shoulder \%MVC Error (\%MVC) } \\
\hline $1 \mathrm{~cm}$ & Average & Minimum & Maximum & $\begin{array}{c}\text { Average } \\
\text { Absolute }\end{array}$ & Average & Minimum & Maximum & $\begin{array}{c}\text { Average } \\
\text { Absolute }\end{array}$ \\
$2 \mathrm{~cm}$ & 0.00 & -0.13 & 0.13 & 0.06 & 0.00 & -0.47 & 0.47 & 0.20 \\
$5 \mathrm{~cm}$ & 0.00 & -0.27 & 0.27 & 0.12 & 0.01 & -0.95 & 0.95 & 0.40 \\
$10 \mathrm{~cm}$ & 0.02 & -0.67 & 0.67 & 0.29 & 0.05 & -2.37 & 2.37 & 1.01 \\
\hline
\end{tabular}

\subsubsection{Time}

Predicted move and reach time difference in all data points, for each level, averaged from under a millisecond for the $1 \mathrm{~cm}$ adjustments to less than $0.02 \mathrm{~s}$ for reach time and $0.03 \mathrm{~s}$ for move times with a simulated $10 \mathrm{~cm}$ input error. The absolute difference from the reference point for the four levels of adjustment is shown in Figure 17. The magnitude and variability of difference from the reference increases with the distance from the reference point (i.e. from 1, to $10 \mathrm{~cm}$ of simulated error). As a percentage of the original value, the response to simulated input error ranged from $0.5 \%$ to $6 \%$ of the original reach or move time value, with the magnitude and variability increasing as the distance increased. Across all levels of simulated error, the effect on move time (with a component in hand) was greater than reach time (with an empty hand). 


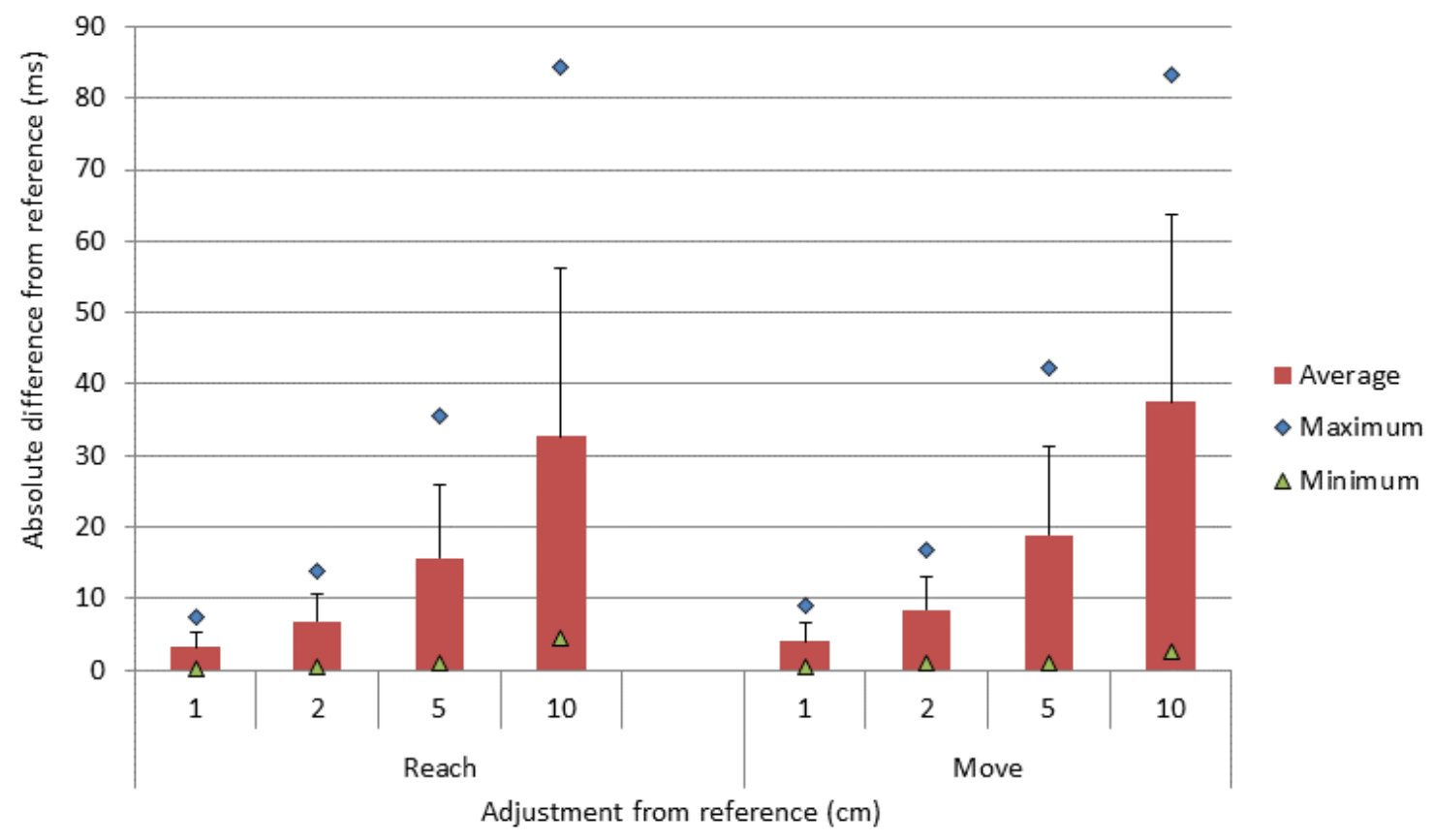

Figure 17. Absolute difference in predicted reach time and move time from the reference time value for the four magnitudes of simulated error tested.

The impact of average user entered hand location errors from Study 2 on predicted move time and shoulder moment is shown in Table 6. Maximum time prediction error based on human input error is $3.59 \mathrm{~ms}$ or less depending on the axis and type of workstation (virtual or physical) being measured. At one standard deviation from the average observed error, time prediction error peaks at $9.90 \mathrm{~ms}$ in the UD direction based on errors in a virtual measurement. Similarly, for shoulder moment, the largest error $\left(-0.02 \mathrm{Nm}\right.$ and $-0.04 \mathrm{Nm}$, for $5^{\text {th }}$ percentile female and $95^{\text {th }}$ percentile male, respectively) occurred in the FB direction when average input error was based on the virtual workstation measures. The magnitude of output error grew to $0.26 \mathrm{Nm}$ when values one standard deviation from the average observed error were used. 
Table 6. Predicted time error (ms) and shoulder moment error (Nm) from average and average $+/-1$ standard deviation (SD) input measurement error (cm) from Study 2 for all three axes.

\begin{tabular}{|c|c|c|c|c|c|c|}
\hline & & \multirow[b]{2}{*}{ Direction } & \multirow{2}{*}{$\begin{array}{l}\text { Input } \\
\text { Error } \\
\text { (cm) }\end{array}$} & \multicolumn{3}{|c|}{$\begin{array}{c}\text { Average Output Error } \\
\text { Shoulder Moment } \\
\text { (Nm) }\end{array}$} \\
\hline & & & & $\begin{array}{l}\text { Time } \\
\text { (ms) }\end{array}$ & $5^{\text {th }}$ Female & $95^{\text {th }}$ Male \\
\hline \multirow{6}{*}{ Average observed error } & \multirow{3}{*}{ Virtual } & RL & 0.02 & 3.49 & 0.00 & 0.00 \\
\hline & & FB & -0.32 & 1.85 & -0.02 & -0.04 \\
\hline & & UD & 0.48 & 2.28 & 0.01 & 0.02 \\
\hline & \multirow{3}{*}{ Physical } & RL & -0.64 & 3.59 & -0.01 & -0.03 \\
\hline & & FB & -0.03 & 2.53 & 0.00 & 0.00 \\
\hline & & UD & 0.44 & 2.14 & 0.01 & 0.02 \\
\hline \multirow{6}{*}{$\begin{array}{c}\text { Average }+1 \text { SD observed } \\
\text { error }\end{array}$} & \multirow{3}{*}{ Virtual } & RL & 0.58 & 3.13 & 0.01 & 0.03 \\
\hline & & FB & 0.84 & 4.17 & 0.05 & 0.11 \\
\hline & & UD & 2.96 & 9.90 & 0.07 & 0.15 \\
\hline & \multirow{3}{*}{ Physical } & RL & 1.27 & 2.35 & 0.02 & 0.06 \\
\hline & & FB & 1.92 & 5.35 & 0.11 & 0.26 \\
\hline & & UD & 2.26 & 8.13 & 0.05 & 0.11 \\
\hline \multirow{6}{*}{$\begin{array}{c}\text { Average - } 1 \text { SD observed } \\
\text { error }\end{array}$} & \multirow{3}{*}{ Virtual } & RL & -0.54 & 3.60 & -0.01 & $\begin{array}{c}-0.03 \\
\end{array}$ \\
\hline & & FB & -1.49 & -1.56 & -0.08 & -0.20 \\
\hline & & UD & -2.01 & -9.03 & -0.05 & -0.10 \\
\hline & \multirow{3}{*}{ Physical } & RL & -2.54 & 1.93 & -0.04 & -0.12 \\
\hline & & FB & -1.98 & -3.34 & -0.11 & -0.26 \\
\hline & & UD & -1.38 & -5.85 & -0.03 & -0.07 \\
\hline
\end{tabular}

Similarly, when the findings of Study 2 were added as error to each of the 84 points of the test data set, all shoulder load outputs, for all percentiles had an average difference within $0.21 \%$ of the original values. Maximum difference from the original values were no more than $0.13 \mathrm{Nm}$ for males ( $95^{\text {th }}$ percentile male), $0.34 \% \mathrm{MVC}\left(95^{\text {th }}\right.$ percentile female), or $2.3 \%$ ( $5^{\text {th }}$ percentile female) of the original value. Magnitudes were dependent on the population percentile being examined. The impact on estimated time was near negligible $(<0.2 \mathrm{~ms})$ within virtual or physical workstations for both move and reach time. The maximum difference observed was $61.2 \mathrm{~ms}$ for a physical workstation. Virtual workstations showed no difference from the reference time value.

\subsection{Discussion}

The simulated and empirical tests to characterize error for the tool has shown that input location measurement error is low and the resulting output error is also low, more so for time prediction than shoulder load. The low average time prediction error is understandable since MTM-1 move 
and reach time prediction has discrete levels in the lookup tables (cf. Freivalds and Niebel, 2009). The discrete levels are either $2.54 \mathrm{~cm}$ ( 1 inch) or $5.08 \mathrm{~cm}$ (2 inches) of distance before the magnitude of reach or move time changes. The impact of location error on time is therefore dependent on the proximity of the location to the threshold for change. Shoulder load values are more variable compared to time because the prediction of load is from a regression equation, which has a continuous effect, and the regression equation is impacted differently depending on input measurement direction.

The low magnitude of shoulder moment output errors from either the addition of the average hand location measurement error to the test data set or the error due to hand load suggests that location measurement and hand load is of minimal concern when the tool is used within its intended scope. In the context of the mean population maximum capability from the 4D WATBAK software (values from Lannersten et al. (1993) and Koski and McGill (1994) ), the maximum hand location error induced difference from the expected value for females was less than $0.36 \%$ of the mean population maximum for arm angles ranging from $0^{\circ}$ to $90^{\circ}$. Similarly, the percent of mean population maximum was under $0.25 \%$ for the maximum difference of the male anthropometrics tested. These magnitudes are much lower than the similarly determined percentages from the shoulder moment change when hand loads are included. Using hand loads up to $200 \mathrm{~g}$ for a $50^{\text {th }}$ percentile male as an example, the average shoulder moment difference is less than $2.3 \%$ of the average population maximum capable for shoulder positions ranging from $0^{\circ}$ to $90^{\circ}$ shoulder flexion. Though hand load errors are a greater percentage than errors due to location measurement, tool, users should not be overly concerned since hand load errors will fluctuate as work elements change. With the tool designed to be applied in seated light assembly hand loads are anticipated to be relatively low and the hand may be unloaded up to half of the time as the worker reaches to get material. It is possible that hand load associated errors could be reduced in future versions of the tool as well. More complex shoulder load prediction models could be incorporated or the general load or specific load prediction models generated to test the impact of hand load could be used. For this reason the equations have been made available in Appendix C for use as desired. Adding more complex shoulder load models may appear beneficial if it reduces error, however, caution is advised. More complex models may require more inputs, such as hand loads that might not be available early in workstation design, and the 
models may generate more outputs that are complicated to interpret without specialized expertise. As a result tool usability could be reduced.

Of the models used in the tool, the \%MVC models are prone to more error. Only a small number of maximal shoulder load capability data sets existed in the literature and were available for use in 4D WATBAK. Subsequently, a maximum comparison value does not exist for each population percentile or limb position when using the technique of Neumann, et al. (2002) to create \%MVC. Having the same maximum value across percentiles means that the \%MVC models are overly cautious for the larger population percentiles because larger anthropometries have a larger limb mass and result in a higher percent of the maximum value. Not having a maximum for each limb position means that a range of limb positions are compared to the same maximum, which creates a discontinuous comparison set. This is reflected in the lower $\mathrm{R}^{2}$ values in the \%MVC regression equations, compared to the shoulder moment regression equations, and is likely the reason for larger tool output errors compared to the shoulder moment outputs.

The test data set used was created to be relative to a real work environment, meaning locations chosen would be at different percentages of an individual's reach capability. As a result, errors for smaller anthropometries may not be truly representative at the extreme hand locations because of an inability of the limb to extend to the point without movement of the trunk. It is possible that this was part of the reason for the percentage differences found between the percentiles, especially when examining the impact of hand load. A future version could guard against overreaching by limiting outputs from reaches not functionally possible. Regardless, in its current form, the tool does provide commentary on excessive reaches by overlaying hand locations with respect to acceptable reach zones. Inappropriate reach distances can be considered a musculoskeletal disorder risk factor when an awkward shoulder posture results (Bernard, 1997).

In light of the results, the small error in this tool suggests that it is usable in industry. As a tool that was collaboratively developed to fit a need during an industry partnership (Greig, et al., 2013b) it fills a gap among other industry standard tools that also have their assumptions and limitations to consider. The strengths of the WEE tool are its application in the workstation 
design stage of seated light assembly, combination of human and system information, and cost efficiency. A large portion of the HF tools available to assess physical risk are observation based (see Takala, et al., 2010) and few incorporate the element of time at all or in a method that connects the tool and analysis to the work system. The use of time in this tool helps to bridge the gap between HF specialists and engineers (Wells, et al., 2007). Compared to digital human models (DHMs) such as Jack, the WEE Tool lacks the comprehensiveness of posture information, such as joint angles, and multi-axial moments. Like DHMs though, the WEE tool is also usable in the design stage, however the WEE tool does not require as specialized of a user and is only a fraction of the cost - traits highlighted by Perez and Neumann (2015) for effective virtual tools. HF tools at the other end of the tool complexity spectrum, tools such as RULA (McAtamney and Corlett, 1993) and OWAS (Karhu et al., 1977), use posture classification to predict risk but are more appropriate for gross motor movements. HAL-TLV (Latko et al., 1997; American Congress of Governmental Industrial Hygienists, 2001) examines repetitive work for the hands, which is more applicable to light assembly. With inputs of hand activity level and normalized peak force, the HAL-TLV, like OWAS and RULA, also lends itself to post-design stage, observation based use. The group of tools that the WEE Tool shares most conceptual similarity with are OCRA (Occhipinti, 1998), EAWS (Schaub et al., 2013), Ergo-MTM (Caragnano and Lavatelli, 2012) and ErgoSAM (Laring, et al., 2005). Common among these tools are that they have a more direct use of work time in analysis, a greater complexity of analysis when compared to tools like RULA, and a typical application in a large assembly environment. Conversely, the WEE Tool is a workstation based, design level tool that can be used to predict work element and cycle time in seated light assembly. We argue that, for a designer considering options in realistic ranges with low loads, or an engineer considering improvement, the good reliability of this tool is more important than additional biomechanical sophistication.

\subsection{Conclusion}

Three studies were completed to test the impact of hand load and location measurement input error on shoulder load and movement time outputs of the Greig, et al. (2013a) Workstation Efficiency Evaluator (WEE) tool. Simulated and observed experimental error created minimal average error in the prediction of shoulder loads $(<0.05 \mathrm{Nm})$ and movement times $(<3.6 \mathrm{~ms})$. 
Potential tool user measurement error was nearly random, and would therefore tend to cancel other errors out. Measurement error averaged within $0.65 \mathrm{~cm}$ of the desired value in both virtual and physical workstation measurements and was more accurate in the virtual measurements. The inclusion of hand load in the predictive shoulder models resulted in predicted shoulders loads greater than the zero hand load base models of the tool, with the magnitude of the difference increasing with hand load and dependent on the population percentile being assessed (average of $5 \%$ to $12 \%$ within the first $100 \mathrm{~g}$ of hand loading and $150 \%$ to $210 \%$ for $1000 \mathrm{~g}$ ). For low level hand loads, the current WEE tool appears to have good capability to predict shoulder load and time within the parameters that it has been tested. The WEE tool can be applied in the design stage of seated, light assembly workstations with little concern for impact of measurement error on tool outputs but has the ability to be improved in its shoulder load prediction accuracy by the new shoulder load models that have been developed. 


\section{Chapter 6}

\section{User-centered development of a workstation efficiency evaluator tool}

\section{Introduction}

This chapter continues the investigation towards objective 2: Develop a workstation level HF assessment tool for light assembly work. The following research question is addressed:

Research Question 2.6 - What are the factors that influence a user-centered design approach to develop a human factors tool for predictive workstation assessment?

The conference paper in this chapter is focussed on communicating the user-centered development process of the tool that was initiated in Chapter 4. It provides context to the development process and demonstrates an approach to developing human factors (HF) tools.

\subsection{Purpose}

This chapter examines how a predictive HF tool was jointly developed through a series of researcher and organizational stakeholder interactions using an action research approach. The research question that guides this chapter is "what are the factors that influence a user-centered design (UCD) approach to develop an HF tool for predictive workstation assessment?”

\subsection{Methods}

The case presented is part of a larger, multiyear case study at an electronics manufacturer. In this case study, we used action research and involved the end-user of a predictive HF tool in the tool development. Action research is a technique that involves cycles of planning, action, and reflection, to reach a goal while, simultaneously, developing an understanding of the process. It is characterized by several elements - involvement of stakeholders in identifying the problem to be addressed, deciding how the problem will be addressed, the research and the collection of data and the joint reflection on the data to guide future work on the problem (Neumann, et al., 2012). Researchers play less of a role of “expert”, transferring knowledge and instead take on a position 
where they engage with the knowledge of those in the setting (Neumann, et al., 2012; Neumann and Village, 2012). Contributors to the tool's development included members of the Ryerson University (RU) Human Factors Engineering Lab research team, company human factors specialists / ergonomists (HFS's), industrial engineers (IEs), product engineers, and their managers/directors, the project manager and the project steering committee - comprised of the researchers and selected senior managers/directors in the organization. End-user involvement included initial development, prototyping, and testing of the tool through eight significant tool design iterations to date.

Data is drawn from both field notes and audio recordings of meetings with representatives from the industry partner. Field notes were recorded in meetings specifically aimed at working on the tool as well as meetings where the tool was commented on during general discussion. Four meetings were dedicated solely to review of the tool. These meetings were usually 30-45 minutes in length and included 4-8 participants. In these meetings, a frank exchange of ideas among members (researchers and a subset of company contributors) was encouraged and occurred. Other meetings included steering committee meetings, where tool updates were presented. In these meetings, with eight to twelve people, a range of topics involving the broader research project were covered.

We expand on the nature of the interactions between the researchers and industry partner (enduser) that guided tool development in the findings below. A general inductive approach was used to understand the significance of statements and actions throughout development. This method searches for core meanings relevant to the research objectives, assembles them into themes that are most relevant to the research objectives and describes the most important themes as an output (Thomas, 2006). The tool development process within this chapter was organized chronologically and three themes were identified based on their occurrence, importance and significance.

The concept of the tool was motivated by discussions among the research team and industry partner. Assessing workstation risk using traditional HF tools, such as rapid upper limb assessment (RULA), a single task assessment approach was ineffective in their electronic components assembly work environment and not sufficiently capturing the low force, high repetition worker demands of the workstation. As the HFS stated, there is "nothing numerical 
that assesses the workstation”. The tool, developed over three years, was intended to assist engineers and HFS's with the evaluation of seated light assembly workstation layout-induced risks to worker health and system performance.

\subsection{Results}

Three themes emerged from the data regarding the development of the HF tool:

(1) HF information needs to be quantified for management of HF - For engineers the indicators of HF are more tangible when they are scored (numerically or colour coded); (2) The tool needs good usability, meaning outputs need to be quickly interpretable and concisely summarized for quick review; (3) Improving the acceptance and integration of the tool required a tool that supports both HF/ergonomic and engineering outcomes. These themes were recurrent within the tool development milestones.

The tool's development has been categorized into eight significant milestones (Figure 18). A milestone is a shift in tool capability or change in data presentation or organization as guided by discussions among the users and the researchers. Tool developments and information that led to changes in the tool during the course of its development are expanded on below.

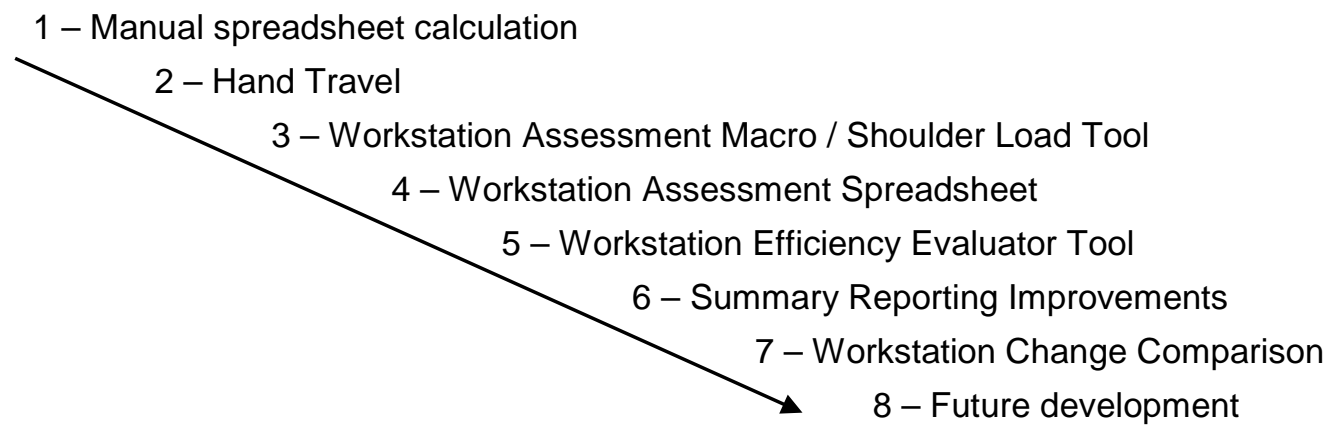

Figure 18. Significant milestones in tool development.

\section{Milestone 1: Manual spreadsheet calculation}

In the first version of the tool, the user had to manually combine information from different data sources. Hand travel, calculated from task element hand locations, was summarized by a spaghetti plot graph. For each element cumulative shoulder load was found using 3DSSPP with times determined using Captiv-L2100. The tool concept was demonstrated by the chapter's lead 
author to the HFS and IEs on a current assembly process along with an example of its application at the workstation design stage. The tool concept was supported as workstation information was needed by an industrial engineer (IE) and the HFS. The first form of the tool was anticipated to be used mainly by the HFS. Providing acceptable reach zones with the hand travel graphs made the idea of hand movement "very graphical" for the HFS and improved results interpretation. However, stakeholders had questions about the applicability of hand travel, return on time invested to get input data, and meaning of the tool's outputs. An IE highlighted the need for outputs with “engineering-like numbers”, which was supported in a later milestone by an engineering manager whose “....work is all about making things quantifiable - give me some numbers.”

\section{Milestone 2: Hand travel macro}

During this development stage, the first program was created - an Excel macro that centralized the tool and data, and automated processing. Inputs to the tool were individual work element three dimensional hand locations, anthropometry (small female or large male) and individual element times. Output tables displayed cumulative hand travel, with hand travel graphs for three movement planes, and shoulder load, calculated from a developed shoulder load model, for each element. The new display of hand travel graphs for all three planes of movement was suggested by the HFS. The HFS appreciated the new graph style, and later in the tool's development added that the graphs prove the point about hand locations clearly and with easy comparison. An IE manager reacted to the tool by saying: "When an engineer is putting his parts here.... you can define your table surface of data and get the $x, y$ coordinates....you could use anthropometric data.... and then you can say this is flexing, this is abduction, whatever." He further added: "This information could be used...for ergonomics risk assessment... [and] could actually develop the cycle time too" which was in line with future planned development by the tool's designer. The IE manager added further that their goal is "really designing stations ergonomically....and to reduce cycle time as well as to avoid injury....And that tool is a very important tool." A senior director saw potential for the tool in the workstation design stage, using CAD drawing information to assess a future production line design. 


\section{Milestone 3: Workstation Assessment Tool / Shoulder Load Tool}

This milestone signified a shift in the tool's application and an evolution in communicating output information. Up to this point, the tool had been frequently called the "Shoulder Load Tool” within the steering committee to promote its ability to estimate shoulder load. However, to better reflect all of the tool's measurement, reporting and prediction capabilities, as well as its use in the design of a future workstation it was rebranded the "Workstation Assessment Tool". Stakeholders who needed more concise report-ready outputs, drove a change in the tool to automatic creation of graphs of results for hand travel and reach zones. This improved the ability of the user to create quicker visual feedback of outputs. The HFS highlighted the need for effective output information that would not overburden the user with large tables of data. Further, the HFS believed an ability to see all data values for each element would always be needed but, in the tool's next iteration a summary should be more prominent with the raw data in reserve. This summary is akin to an "executive summary,” which was developed and refined later in the tool's development under the guidance of an engineering director.

\section{Milestone 4: Workstation Assessment Spreadsheet}

The tool structure shifted from being Excel macro-based to a series of linked spreadsheets. The rationale behind this change was to 1) avoid potential macro-related security concerns (thus improving usability); and, more importantly, 2) allow real-time feedback of input changes in the output graphs, tables and summary. This program change allowed the user to more easily try “what if?” layout scenarios. A new summary page highlighted the results, which included scoring element locations based on reach zones. Support information (calculations and guidelines) was hidden to make the volume of information less imposing. Reach zone scoring enabled the creation of an aggregate value describing hand location for an individual arm. The introduction of movement and process time estimation via methods-time measurement (MTM-1) description of elements was considered a step towards estimating physiological requirements similar to what had been seen by the company HFS in heavy manufacturing. At this stage in its development, the tool was seeing increased use, but was found to need instructions to help users. 
Milestone 5: Workstation Efficiency Evaluator (WEE) Tool:

In this phase of the tool's development, the user interface was further improved and instructions for use and explanations of summary outputs were added. The summary sheet was enhanced by a table of highlight information. The tool was rebranded the "WEE tool” because of the additions of time prediction, allowing commentary about efficiency of work processes, and recovery allowance prediction and also because of continued interest by the steering committee for fatigue prediction in an attempt to estimate worker efficiency. A continuous improvement prioritization metric was created to rank elements to examine for continuous improvement - a common management theme. The metric is intended to inspire tool users to work beyond red, yellow, green target thresholds and strive for the best workstation layout possible. The WEE tool usability and relevance was tested when it was used to review a new line setup and any subsequent changes. The improved number and quality of output measures was perhaps starting to justify the effort of input data collection. The tool's applicability to workstation development was evident. A statement by the project manager compared the science behind the tool to previous methods used for workstation design "I don't think that there is this kind of science behind it [the previous method].”

Milestone 6: Summary reporting improvements

The summary table was reorganized as requested by an engineering director. The follow-up review was positive "I like that prioritized ranking too... you stuck that summary right at the top.” With the top three continuous improvement priority items placed at the top of the summary the engineering director pointed out "Our life is all Pareto. It's going to be top 3, top 3, top 3." Input elements were classifiable as "Value Added" and "Non-value Added" which supported the organization's lean principles. A reworked summary page highlighted key values and processes and provided an executive summary of the workstation. A secondary summary page provided details on continuous improvement components for each element allowing engineers to drill down to better understand issue causes. With this summary design the engineering director was able to see the tool's use; “... the reason we also have the red, yellow, green here is that we're saying 2's [reds] aren't allowed. Period. We've got to fix the 2's[reds]....The summary table gives me the aggregate worst ones overall and then the next tier for my team is then to come in 
and say you have to get rid of all the reds. Because we're saying 2's aren't allowed to the HF scoring tool". Further, the improvement in HF via the tool was evident as well "....I think the feedback from my team has been very positive too.....objective feedback like this.... is much more effective than it has been in the past....I'm certainly supportive from above". This support was demonstrated by the manager by arranging a meeting to share the tool with the industrial engineering group, who the manager believed would "see the value in this as well" as the tool would give the IE "team the ability to provide more back to the system". A product engineering group was also showing interest for application of the tool in their early system design stage. Sample results were posted on the line by a product focussed engineer to demonstrate the use of a tool output as part of their innovation in design. Tool input interface improvements were also suggested by the HFS to allow data entry to be more intuitive.

\section{Milestone 7: Workstation change comparison}

The input and output information that was once spread across multiple worksheets was combined into one sheet, a necessary step to allow for a comparison between two workstations. Having a tool that had the ability to compare workstations was a necessity for the industry partner. A single-sheet comparison summary highlighted the percent change occurring in the comparison which was a desired output from the users. The HFS was using the tool more frequently in this development phase and starting to understand the tool's ability to estimate time. Hearing of the potential to estimate time from digital drawings, a product focussed engineer was eager to trial the tool early in the process development stage to estimate time of assembly.

\section{Milestone 8: Future steps}

More HF measures are being added to strengthen the HF presence because of the recent focus on the system output side of the tool. In an earlier review of the tool by the RU research team, a member stated “Where has the HF gone?” Cumulative shoulder load and improved logic for recovery allowance are being implemented in the tool. The summary comparison of key measures (e.g. movement time, reach zone count, hand travel) in a comparison between two workstations has been modified to colour code the type of improvement - red, yellow, green. The HFS has integrated the tool into their tool box and has promoted the tool as a measurement method in ergonomic development projects. 


\subsection{Discussion}

This case study demonstrates how a joint UCD approach with users' and researchers' input was used to create a customized tool for adoption within the partner organization. The AR approach, coupled with UCD, provided an opportunity for discussions between RU researchers and organizational stakeholders that shed light on the type of information desired and the work processes that the tool could apply to. Analyses of the discussions highlighted three themes within the tool's development. The findings highlighted the importance of quantification of HF, tool usability, and output information simultaneously supporting both HF and engineering interests. The need for quantification of HF information to improve understanding and management of HF is not surprising since a longstanding belief in business management is that “you can't manage what you can't measure”. The addition of system relevant, engineering measures (e.g. value added classification and time estimation), alongside HF outputs, and the structure of the quick summary display of information were necessary to connect the HF information with the engineering groups and align stakeholder interests. This finding is similar to the findings of research by Waterson and Kolose (2010). The development of predictive HF measures helps to close the gap on the need for leading indicators to predict outcomes and manage worker and system risks, a suggestion for improving HF integration into work system design (Neumann and Village, 2012).

Not all developments of the tool were able to be delivered or were positively reviewed. The steering committee was very interested in finding a method to estimate and scale worker efficiency based on task demand. That type of assessment stretches the current capabilities of fatigue estimation and worker performance beyond current capabilities and requires further research. One challenge facing the tool is ownership in the organization. The tool is designed to span HF and engineering boundaries, meaning it spans knowledge bases and responsibilities as well. Ideally, anyone can use the tool, however the HFS will need to continue to champion the tool until knowledge and understanding of the tool increases and its integration within the design process matures. 


\subsection{Conclusion}

The chapter demonstrates the ability to use UCD and AR to develop a predictive HF tool to assess light assembly workstation layout. Contributions to the development of the tool by engineering groups, HFS's and researchers have helped the tool to evolve so that it combines system performance and physical workload information in its outputs. The coupling of system and worker information allowed the tool to predict aspects of worker efficiency at the workstation design stage. Examination of the development process identified three influential tool design factors: (1) importance of quantification of HF; (2) tool usability; and, (3) tool output information that simultaneously supports and connects HF/ergonomic and engineering interests. These factors could be applied to help future HF tool development. 


\section{Chapter 7}

\section{Assessing organizational human factors integration and capability}

\section{Introduction}

This chapter addresses objective 3: Create a tool that reports the level of HF integration and maturity in an organization, and includes the following research questions:

Research Question 3.1 - How can the level of HF capability and integration maturity in an organization be assessed?

Research Question 3.2 - What are potential user views on the contents of the tool developed in $R Q 3.1$ ?

Research Question 3.3 - What are users' views on the content and usability in a case field trial of the tool developed in RQ 3.1?

The focus in this chapter is on the collaborative development of an organization assessment tool. Feedback from the case organization as well as participants in workshops used to validate the tool are incorporated into the tool's design. Themes describing the reaction to the tool were identified using qualitative analysis of the interactions.

\subsection{Aim}

The aim in this chapter is to present the development and evaluation of a tool that can 1) assess the state of HF capability and integration in an organization, and 2) help organizations to know what the ideal HF capability and integration within an organization might look like. This will be addressed in the chapter in three phases, which include theoretical development, workshop validation, and field testing of the approach to assessing HF integration and capability in an organization.

\subsection{Methods}

An action research approach was used to facilitate the participatory tool design. With this approach an ongoing exchange occurred between a Ryerson University research team and an 
industry partner to develop and test the tool. The interaction was part of a larger research project investigating the integration of HF in the design of production systems (Village, et al., 2014a). Experience within the Ryerson research group included approximately 150 combined years of research and practice in fields such as ergonomics, biomechanics, engineering design, sociology, industrial engineering, and health and safety. The industry partner was an international electronics design and manufacturing company. Industry contributors $(n=8)$ were part of the product realization business unit of the company. Engineers, managers, directors and HF specialists within the company provided feedback on the tool concept, early content and layout, and executed field testing.

\subsubsection{Conceptual development}

The tool development approach combines concepts from audits and performance measurement and management systems. The assessment in the tool differs from a traditional audit and assessment (e.g. ISO, European Foundation for Quality Management (EFQM)),or ergonomic self-auditing process as suggested by Bierwirth, et al. (2010), in that it is not an assessment of prescribed processes or specific practices. This was an intentional choice in order to allow flexibility to apply the tool in different organizations. The scope of the tool covers human factors (HF) as outlined by Dul, et al. (2012) and defined by the International Ergonomics Association (2014). The tool is intended to cover all parts of the organization due to HF being a system approach, the suggestion by Wilson (1994) that ergonomics should spread throughout an organization, and that the incorporation of HF throughout an organization can have system-wide benefits (Goggins, et al., 2008; Dul and Neumann, 2009; Rose, et al., 2013).

The tool is therefore guided by the following principles:

1) HF is a means, not a goal.

2) HF applies to all aspects of an organization.

3) The tool should not be prescriptive, allowing for different approaches in different contexts.

4) Scoring should be a range from 'nil' to 'ideal' on a simple 0-4 scale.

5) The tool should be a self-administered organization assessment via interviews with key informants. 
Accomplishing these principles required the division of a generic organization into distinct analytical units, defining processes and outcomes that contribute to the ideal HF within the units, and identifying distinguishable levels of maturity for the processes and outcomes.

Organization analytical units were termed as functions in an organization. A function is defined as a role that exists in the organization, for example the role of health and safety, or product design. The term function was chosen over department to avoid excluding small organizations where distinct departments might not exist but instead a single person may have responsibility for a multitude of functions in their organization.

An HF element is considered to be a process or outcome that contributes to the achievement of the ideal HF for the function. It could be something measured, reported, a method installed, or a mindset within an organization to achieve that ideal state. Each HF element poses a single item to be evaluated.

Based on the preceding principles and definitions the framework in Figure 19 was used to guide the tool development. In this framework the organization was broken into core functions. Within each function there exists a range of HF elements that can contribute to the ideal HF. The HF elements would then be scored for the HF integration and capability maturity based on a guiding rubric. Summing the scores for all HF elements generates a score for the function. The scores from all functions can then be reviewed to gain an appreciation of the HF capability and level of integration in the organization. 


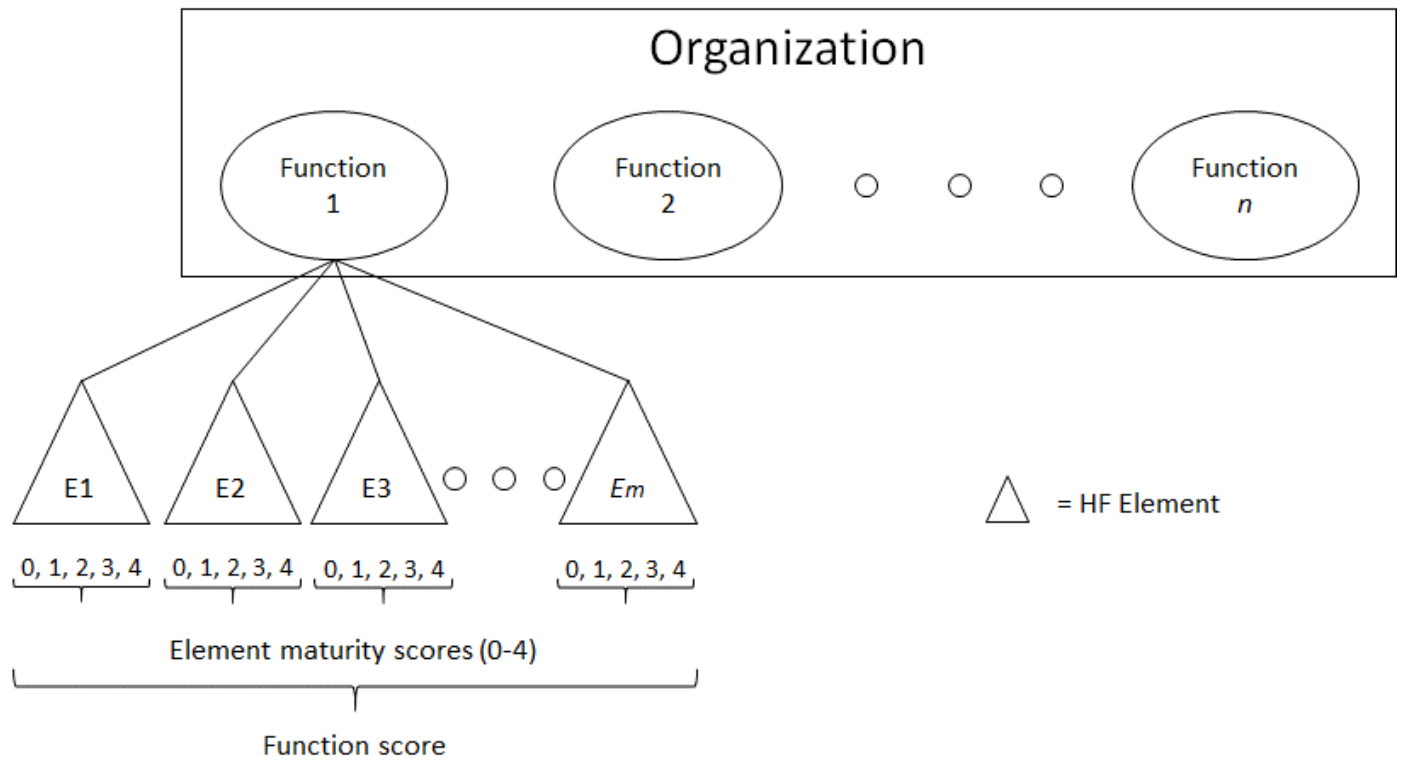

Figure 19. Framework for the development of the tool, where $n$ functions exist in the organization and $m$ HF elements are contained within a given function.

A consensus building approach informed decisions on content in the first draft of the tool. The research team identified, and reviewed for consensus on applicability, titles for key functions where HF would be relevant in an organization. The relationship to the organization of each chosen function was then defined, as was the ideal HF situation within the function. These definitions were necessary to focus the scope in the remaining development steps and to align future tool users with the context with which the function was considered. The ideal HF for each function was defined based on key themes or requirements for ideal HF as identified in the literature and through past field experience of the research team. Ideal HF means HF that is not only the best for the organization or its industry peers but the best regardless of the industry. . Based on the ideal HF in each function, and again supported by experience and research literature, HF elements that contribute to the ideal for that function were identified. All identified HF elements for all functions were grouped and examined for overlap between functions. Similar elements were combined under a common theme to minimize the total list of HF elements. For each HF element within a given function, a statement reflecting the ideal HF was created for that HF element as it related to the function. This was considered reflective of 'ideal', mature HF integration and capability. 
Literature seeded the development of definitions of the HF ideal. A number of themes were found to guide the definitions of the HF ideal for each function and the HF elements supporting the functions. Evident themes included:

- HF has been suggested to take a systems approach, be design driven and combine performance and well-being (Chapanis, 1995; Dul and Neumann, 2009; Neumann and Dul, 2010; Dul, et al., 2012; Wilson, 2014).

- The impact of HF needs to be measured and reported in a manner that is understood by stakeholders and relates to company key performance indicators, strategy, and business goals to better manage HF, improve its utilization, and support stakeholder and organizational success (Rodrigues, 1999; Fulton Suri, 2001; Dul and Neumann, 2009; Dul, et al., 2012; Edwards and Jensen, 2014; So and Lam, 2014; Village, et al., 2015).

- HF measures need to address direct and indirect costs, return on investment, cost-benefit analysis, and compensation claims so that $\mathrm{HF}$ is viewed as an organization investment and not a cost (Wilson, 1994; Hägg, 2003; Goggins, et al., 2008; Rose, et al., 2013).

- The application of HF should be throughout an organization, from strategy development to product development and the associated processes (Perrow, 1983; Chapanis, 1995; Stanton and Stammers, 2008; Dul and Neumann, 2009).

- The HF implementation, integration and HF program should be evaluated regularly (Kragt, 1995; Bierwirth, et al., 2010).

- Some initiatives need to have an HF specialist integrated but should always employ a participative approach and get everyone involved (Wilson, 1994; Kragt, 1995; Wulff et al., 1999a; Wulff, et al., 1999b; Vink et al., 2006).

- Reviewing HF components of a product or process should consider not only the physical aspects but the psychosocial and cognitive aspects as well (Fulton Suri, 2001; Hägg, 2003; Wilson, 2014).

- User-centered approaches should be used and include an empathetic view towards the people involved during design, review and implementation (Eason, 1995; Fulton Suri, 2001).

- Ensuring high quality HF is further dependent on continual HF training and learning and providing guidelines of relevant context for non-specialists (Chapanis, 1995; Dul, et al., 2012; Hall-Andersen and Broberg, 2014). 
To create a scoring range within the tool, the ideal HF element was scaled across four other levels of maturity, each level having its own descriptive statement. This continuum, bookended by statements for the best and worst case scenarios, was conceptually similar to the levels of the Baldridge Performance Excellence Program (National Institute of Standards and Technology, 2012). Scores, assigned to each level to reflect a 'nil' to 'ideal' scenario, ranged from 0 to 4 , with 4 representing the ideal scenario. A score for a function was obtained by summing all individual element scores and dividing it by the total score possible.

\subsubsection{Workshop Validation}

Three workshops were held to review tool content (i.e. that the tool includes the information that it should) and face validity (i.e. that the tool does what it appears to) (Trochim and Donnelly, 2007). A convenience sample of participants (8-20 participants per workshop, 45 total participants) were recruited from professional societies and graduate programs of the fields of ergonomics, industrial hygiene, health and safety, and industrial engineering. One workshop recruited ergonomists from the Association of Canadian Ergonomists, another workshop recruited industrial engineers from the local chapter of the Institute of Industrial \& Systems Engineers, while the third workshop was completed in a graduate ergonomics course that included industrial hygienists with a range of professional experience. Workshops were structured to guide the participants through the tool development process and the background, supporting information for sections of the tool. Four functions were reviewed in-depth per workshop. Breakout groups allowed the participants to simultaneously review and develop their own sections of the tool for comparison with, and validation of, the current tool. Participants were asked to provide comments and feedback at the end of the workshop using a seven point Likert-type scale. A one-sample t-test with 95\% confidence interval tested the null hypothesis of an opinion neutral midpoint on each of the five following statements about the need for the tool and the appropriateness of content and tool design:

1. The HFIT is a tool that is needed.

2. The functions chosen in an organization are appropriate for the HFIT's scope.

3. The element themes are appropriate and significantly cover human factors and ergonomics concerns. 
4. The definition of World Class, or ideal HF are appropriate and accurate for the tool.

5. The reporting format is user friendly and easy to understand.

The presentation of information and general discussion questions were audio recorded for postworkshop verification of any key comments. Participants were also instructed to write down information and ideas from brainstorming during small group work. This information was collected for review after the workshop. All comments and information were reviewed and used to improve the tool.

\subsubsection{Field testing of the tool}

HF specialists in the partner company were recruited to use the tool to assess the level to which the organization considers human factors in its activities. The tool was presented to the HF specialists, and a high-level explanation given as to how it was to be used. HF specialists recruited subject matter experts they deemed appropriate when assessing the different functions in the tool. The specialists facilitated the use of the tool with the subject matter expert(s), noting comments about the usability and content of the tool. Researcher assistance was provided as requested, otherwise the HF specialists were left on their own during the use of the tool. During a number of assessments, the researcher observed the process of use of the tool, noting comments about tool usability and content. After each scoring, and, again when all function assessments were completed, the researcher and HF specialist(s) met for a semi-structured interview on key development and experience themes about the tool. The discussion was guided by questions on the user experience, the appropriateness and scoring of content, gaps in information, application of the tool and use of the outcome information. With permission, discussions were recorded to verify accuracy of comments. Research was predominantly qualitative, using guided discussions, field notes and audio recordings of discussions that were transcribed as required, as well as written feedback on the user's experience with the tool. Field notes from all interactions involving discussion of the tool were reviewed for any emergent themes using the inductive approach of Thomas (2006) and, specifically for issues around reaction to the tool, its application and design improvement suggestions. 


\subsection{Results}

\subsubsection{Conceptual development results}

The complete, initial document included definitions of all functions and HF elements to provide context for the user, scoring sheets for each HF element in a function, and summary scoring tables for an individual function and all functions combined. The tool was initially titled the Human Factors Integration Tool (HFIT). An example summary scoring checklist and associated questions for scoring for the Training function can be found in Appendix D.

Research group discussion generated a list of 16 functions. These included:

1. Environmental, Health \& Safety

3. Training

5. Tooling

7. Medical Services \& Claims Management

9. Marketing/External Communications/Advertising/ Retail/Sales

11. Finance

13. Maintenance

15. Human Resource Management (Hiring and Retention, Employee and Labour Relations and Internal Communications)
2. Construction and Fabrication

4. Operations/Supervision

6. Product/Service Design

8. System Engineering / Design

10. Logistics

(Shipping/Receiving/Material Handling/Warehousing/Storage)

12. Scheduling/Operations Planning

14. Quality

16. Organizational Strategy Development (Board of Directors/Senior Management)

Two examples of definitions of the ideal HF for a function are shown below for the functions Training and System Engineering / Design.

\section{Training:}

Training modules are reviewed and compliant with promoting effective HF practices (well-being and performance). HF awareness module is used and employee introduction training is completed with frequent refresher. HF information in training has both general, but simple HF information component, and a component that is context specific to the organization. Role specific training provided to front line employees, managers and system designers.

\section{System Engineering / Design:}

HF inclusion as a key contributor with the equivalent power to stop a process and force revisions to the design. All modifications are automatically considered for HF impact and interaction without prompting. Lessons learned from other experiences feed into any 
new design. All HF concerns addressed while in the digital/development stage. Layout allows flexibility to cover entire working population.

A list of 31 discrete HF element statements were created and structured to improve the number of common terms between functions (see Figure 20). Two HF elements were added to the original 29 proposed elements, along with modification of language within some of the original elements, as part of a revision requested by the industry partner in order to better allow for the assessment of knowledge workers. An average of 10.2 functions were served by a single HF element $(\min =2$, $\max =16)$. The average number of elements per function was $19.7(\min =12$, $\max =26)$.

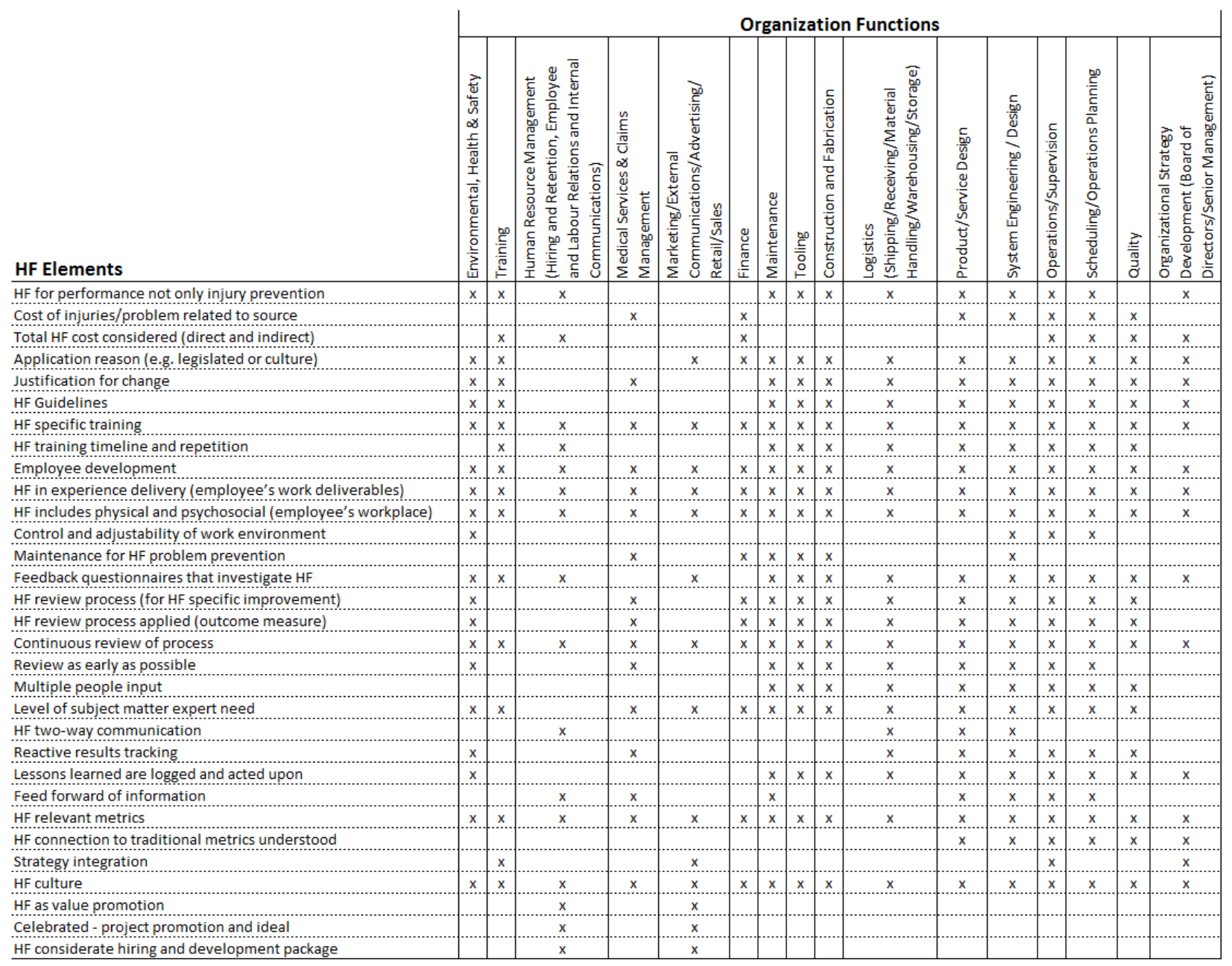

Figure 20. List of all Organization Functions and the corresponding HF Elements within each function (indicated by an 'x'). 
An example of three elements and their HF ideal are shown below:

\section{HF Guidelines:}

Refers to standards and recommendations for procedures and outcomes. Guidelines are industry based or established by a subject matter expert. They are reviewed frequently for relevance and tailored to an individual organization to increase understanding.

\section{Lessons learned are logged and acted upon:}

HF related lessons (e.g. from projects or improvements) are logged and circulated among key people to support continuous improvement. The information exist as a living document space that is updated regularly and provides continual feedback to the next lifecycle of service or product.

\section{Celebrated - project promotion and ideal:}

Successful HF specific projects are celebrated within the company and promoted. Ideas brought forth by employees are noted and published. The culture of HF and desire to be a World Class HF leader is evident in the organization.

An example of the rubric created from the HF ideal for HF Guidelines is shown in Table

7Error! Reference source not found. for the Training function. Users would choose the statement that best applies to their situation to determine the appropriate score.

Table 7. Example of an assessment rubric for the HF Guidelines HF element of the Training function.

HF Guidelines (\#6):

\begin{tabular}{l|r}
\hline Level of Human Factors Maturity & Score \\
\hline HF training does not include HF guidelines. & 0 \\
\hline HF training occasionally includes generic HF guidelines. & 1 \\
\hline HF training frequently includes generic HF guidelines. & 2 \\
\hline HF training occasionally includes organization specific HF guidelines. & 3 \\
\hline HF training frequently includes organization specific HF guidelines. & 4 \\
\hline
\end{tabular}

Comments:

\subsubsection{Workshop validation results}

Workshop audiences included subject matter experts from local chapters of ergonomics and industrial engineering associations, and graduate students. Participants had backgrounds from manufacturing, consulting, government, health care, meat processing, oil and gas, and process improvement, among others. Comments on the tool were, at times, unique to the specialities of an individual workshop, but, at other times, did reflect a commonality across all subject matter experts. Participants on average rated the need for the tool (see \#1 in Figure 21) at 5.7 on the 7 point scale $(\mathrm{n}=45, \mathrm{t}(44)=10.993, \mathrm{p}<0.001)$. Associated positive comments included “I think 
this is a revolutionary tool and I haven't seen anything like this", that "this is quantifying something not previously quantified”, and that "it's needed so long as there are enough personnel that are capable/knowledgeable about HF to use it." Some were "unsure if companies are ready...", that the tool "seems to be useful to more advanced companies (overall scope)" and could "see it(s) use but wonder how effective it would be on a wide scale."

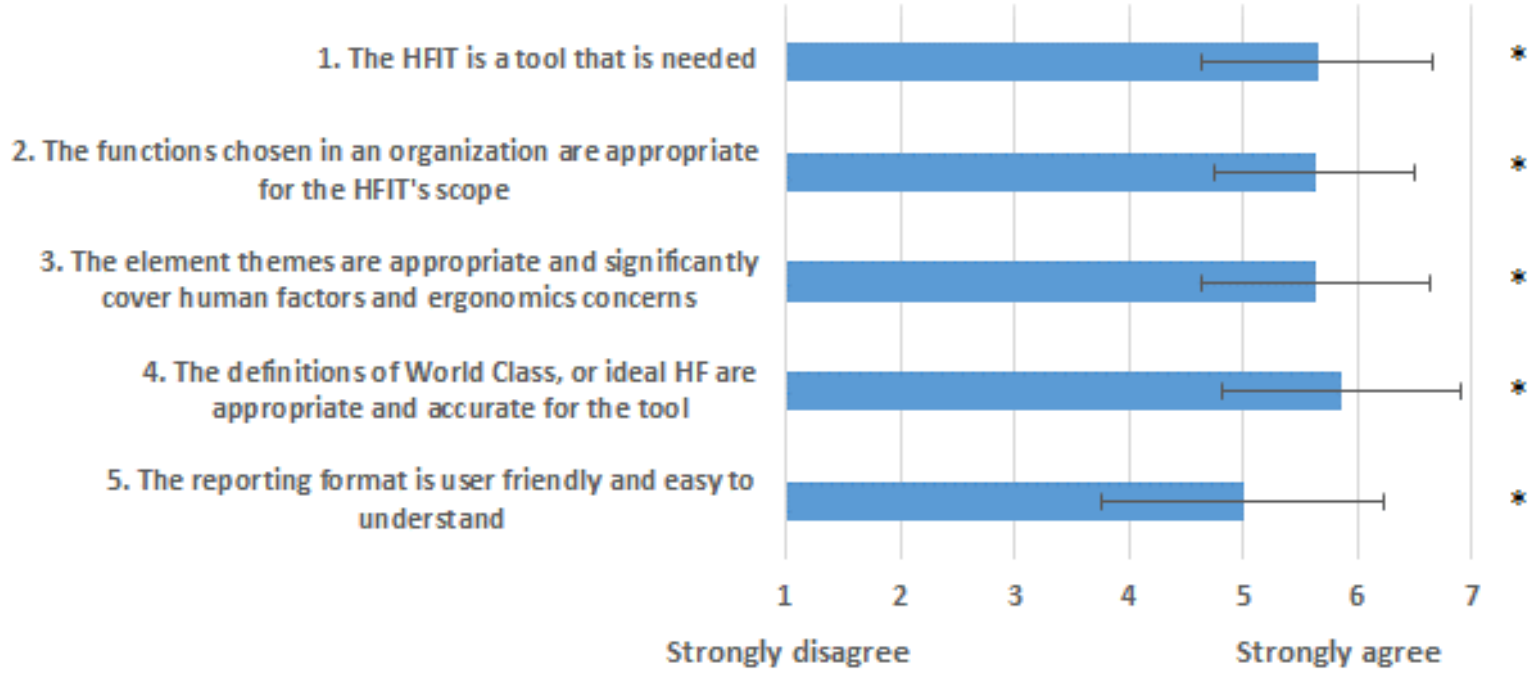

Figure 21. Workshop participant feedback (Average +/- 1 SD; 42 to 45 respondents per item; * denotes significantly different from 4 , a neutral agreement $(\mathrm{p}<0.001)$ ).

Participants suggested that research and development and information technology were two functions to also include. Regardless, workshop participants agreed $(5.6$ out of $7, n=43, t(42)=$ 12.221, $\mathrm{p}<0.001$ ) the functions were appropriate for the HFIT's scope (see \#2 in Figure 21). Some participants thought some functions already included could be expanded on, be "more industry specific" and "include some other health care functions." Others thought that the functions "seem to make sense" and that the "functions will become better defined with more exposure in industry." Comments on HF elements were also positive, though an assessment by participants was difficult to make in the workshop due to the volume of material and the associated time to complete reviews. One participant did suggest that they "would like to see more behavioural aspects of human factors" and another stated that we need to "ensure that (the) user understands human factors applications to ensure accuracy of reporting/rating." The idea of World Class HF (see \#4 in Figure 21) was the most agreed with feedback statement (5.9 
out of $7, \mathrm{n}=43, \mathrm{t}(42)=11.765, \mathrm{p}<0.001$ ) but left one participant to wonder if we can”...ever really achieve World Class...." and another to state that "because no standards exist for application of HF it's hard to define world class"; however the participant followed that statement by adding that World Class "is defined well for the tool though." The least agreed with feedback statement (\#5 in Figure 21) regarded the reporting format (5.0 out of 7, n = 42, $\mathrm{t}(41)=5.270, \mathrm{p}<0.001)$.

Five themes evolved from the comments. These included information clarity, tool length and volume of information, differentiation of information, and use of an electronic format. Some suggested the reporting clarity was concise and clear while others stated it could be confusing and needed explanations and specific examples. Others commented that the terminology and content was "geared towards educated individuals" and "not friendly at all” and more easily understood by ergonomic or HF specialists. Similarly, some participants commented on the need for more clear section divisions and that some users may have "trouble differentiating the different score categories.” The volume of information was considered daunting and long and that future versions of the tool should consider an electronic format.

\subsubsection{Field testing results}

Sixteen documented dedicated meetings or informal conversations were held on the HFIT in the partner company. Five functions, with six total subject matter expert participants, were able to be tested in the field (Environmental, Health \& Safety, Medical Services \& Claims Management, Product/Service Design, Marketing/External Communications/Advertising/Retail/Sales, and Human Resource Management) with researcher participation in three of the sessions. Attempts made to test other functions were unsuccessful due to resource and personnel constraints in the company. Comments from the interactions are clustered into three key themes representing the need for the tool, the reaction to the HFIT, and tool design issues.

\subsubsection{The need to measure HF across an organization:}

The need for a method to assess ergonomics in an organization was evident from participant comments. After an exercise of rating the organization's HF level of integration, one engineer commented "I would like to get to 10 and I don't know what 10 looks like and that is why we need your help." An engineering director commented in a separate meeting on the desire to be able to show how the impact of multiple human factors projects could be demonstrated to their 
leadership team - "[We] can demonstrate why we're doing things.... We can say this is why we're choosing this project. There [are] benefits for it but we also have to grow in this area. This is an area we are weak in."

\subsubsection{The reaction to the HFIT}

The introduction of the tool to the HF group was positive, and appeared to fill a need and be appropriate in scope. One HF specialist stated "I would love to see this applied...across our entire organization...it's just going to keep moving, in terms of overall, the evolution of ergonomics." Another HF specialist believed "any tool where [you] can ask pertinent questions to introduce [people] to ergonomics is good....I find the information is fine.....I think it's general enough where the person using it can make some adjustments for the work that's being done in your place." Similarly, an engineering director was also supportive "...I like it. I think the grades are good...[it] is a great evaluation tool for me."

An HF specialist highlighted the challenge with this being applied in other industries where there is "still heavy injury focus with most people", though they thought that in large manufacturing (e.g. automotive assembly) HF is seen differently so the tool may apply there sooner. The HF specialist believed that the tool would not be picked up and used by just anyone, especially if they did not see the value "in human factors as a whole" and make the connection to their work "if it wasn't related to anything that helped them make their job easier or quantify stuff or improve, whatever their service or product was." They believed this was in part due to the complexity of HF, people's general knowledge of HF and not completely a fault of the tool. Even for those with some familiarity of ergonomics they might not understand the connection with human factors. Two HF specialists commented that even the term ergonomics would be more well-known than human factors in their organization with one of them stating that there would be a need to "be sure that people have a good foundation of understanding of what human factors is and what this is designed to accomplish to make sure that they receive the results.....I can see the context might be misinterpreted." As a result, one HF specialist believed that in its current form the tool is best used by ergonomists, in part because “...I have difficulty [explaining] ergonomics to people other than injury prevention....I think most people understand the physicality of work.....looking at it from a productivity standpoint they don't....grasp that because I don't think in their mind they are able to measure that kind of thing." The tool was 
also highlighted to have benefits beyond the case organization as a tool for consultants proposing work, shown when one HF specialist commented “...as a consultant it would be an extremely beneficial tool. To be able to go in and say 'Look. As part of our services we will do a survey.... an audit up front and when we're done you should have X\% improvement'.”

\subsubsection{Application and benefits of the tool}

Different stakeholders envisioned different applications of the tool. One engineering director thought its application could be similar to lean assessments, where effectiveness is evaluated across industries and organizations. HF specialists saw application in understanding the current state of HF in the organization, developing conversations around HF, and planning projects to further develop HF capability in the organization. When establishing the current state of HF, one specialist thought the tool could help to change the culture of HF beyond focussing solely on well-being "to talk about human performance and efficiency" as the company transitioned to a more lean workforce. This specialist had found it "hard for me to push [the performance agenda] up the line" but that "ultimately if we can show the company that integrating human factors and getting everybody to four, that's where you're going to have your most success in eliminating the conditions that would prevent people from performing." In this way, it was possible to use the tool to develop conversations with groups in the organization who the HF specialists were "not sure how much they see the relation [to HF]." The view of an HF specialist was that they were "for the most part...on the outside looking in" but with the tool they now have a "means to quantify where that is for us", giving other stakeholders "some incentive” to change, as well as provide a means for the HF group of selling their services.

Regardless of the score from the tool, the HF group perceived there was good output. One HF specialist illustrated the benefit when they commented "We would benefit from this in some way whether it's the actual score, [or] whether it's ...having those discussions." With experience, the one HF specialist "started to figure out how to use [the tool]." They recognized that "each one of these [spreadsheet] tabs...in themselves...is a project" and began to plan projects to improve the HF capability as they started to realize "there were deficiencies and/or good things coming out of the tool and understanding that this is where we want to go from an ergonomics program perspective that helped shape it into a project." Building on this perspective they commented how the results inspire projects that are guided by the information within the levels of maturity of 
the HF elements and enable discussions to move HF forward; "I feel like the questions itself are enough to say 'Okay. You're at this level. You know you want to go to this level. Here are some metrics to show you the difference and these are the things that we're going to do to get you there'... as opposed to now where we don't even know where that is and how to get to the next point." In this case, the tool would also help to assess and audit their change progress through the levels of maturity. The scores and the statements on HF maturity could also help to provide a guide to change and a method to quantify that change. This had been previously unavailable at the organization level.

\subsubsection{Tool design issues}

Development ideas were shared with the industrial partner during stages of development for feedback. Issues on the choice of elements, clarity of language in the document, layout, the addition of information to capture knowledge workers, and the addition of an IT function were highlights of the views on the tool. Design issues were not always consistent among stakeholders. For example, though an engineering director wanted more specificity to statements on frequency (e.g. specific definitions of rarely and occasionally), an HF specialist was comfortable with the frequency terms as they were because they used their professional experience from creating physical demands description reports as the basis for how they interpreted frequency.

Discussion with stakeholders about the concepts and the idea for the tool led to an initial version far different from what is currently in place. The adoption of functions and HF elements with multiple levels reflecting HF maturity coincided with comments of an engineering director to put more thought into the basic contributing elements used in the initial version of the tool. The resulting large document created, understandably, a concerned reaction amongst HF specialists that was similar to the workshop participants. The tool appeared to be received as more manageable when the components of the document were broken down into short sections (i.e. the individual functions) which could be completed in 30-60 minutes each. Even when working with the individual functions, the tool layout was considered "clunky" since the HF specialists had to switch between pages for definitions and descriptions. The creation of a digital version, both pdf and an automatically tabulating Excel based version, addressed this deficiency. 
A "large chasm" between the two highest maturity levels was perceived by one HF specialist though a value between three and four was not considered a solution. They believed that users need to "complete that [level] to get to the next level" and that "[People] are going to have to read the question to be able to understand... what it would take." In their opinion, providing guidance on making the transition between levels, such as the "No, Some, All but minor, All but major, All in major" within the tool, would prevent users from unrealistically choosing the higher number.

Further development suggestions focused around improving support information as well as improving the clarity of the language in certain elements. This included the need for a thorough explanation of what $\mathrm{HF}$ is and its relation to the person being interviewed. In elements tested in the field, it was evident that this tool may not be straightforward enough to be used by non-HF specialists, or even in different industries. Improving the ability for people to "conceptualize and understand" the tool and "figure out how it would be applied" was highlighted in order to "get that ramp up quicker" to be able to move forward. One stakeholder that was interviewed for a function commented on the need to "make it as simple as possible. Dumb it down. Do whatever you can" because they had been simultaneously figuring out the tool and recounting earlier answers as the discussion moved along. Use by non-HF specialists could be potentially addressed by providing more support documentation to educate the user, while, as suggested by an HF specialist, plainly explaining the benefits to the company and perhaps supporting the benefits with cost savings and statistical information could help to improve interest.

\subsection{Discussion}

The Human Factors Integration Tool (HFIT) has been developed to allow users to better understand the HF-related capabilities existing in their organization and the level of integration and maturity of these capabilities relative to a defined world class ideal. The tool does not specifically prescribe what should be done in an organization, but instead provides guiding HF

elements as defined by subject matter experts using a consensus-based approach. These guiding principles are expected to benefit employee well-being and organizational performance. In the Authors' view, this principle approach, and the use of functions instead of departments, allows the flexibility to apply the tool in organizations of different sizes, and perhaps transfer to industries beyond manufacturing as well. Though not an audit, the tool provides a 
comprehensive assessment of HF performance execution and process knowledge in an organization, which makes this unique when compared to other HF tools which provide knowledge of performance outcomes (for example Takala, et al., 2010).

Although the attempted comprehensiveness of the HFIT makes for a large document which was of some concern to Stakeholders, the review time of 30-60 minutes per function and total estimated review time of 8-16 hours is comparable to occupational health and safety management audits reviewed by Robson et al. (2012). Regardless of concerns, HF specialists working with the tool highlighted its ability to initiate conversations with other organization stakeholders, a similar finding in engineering tool adaptation by Village, et al. (2014b) and also speaks to the boundary spanning needs within HF identified by Garrety and Badham (1999). These conversations can help to demonstrate that HF extends beyond health and safety and connects to the performance of the whole organization, both in short term performance disruptions and in the loss of expertise from long term injuries (Rose, et al., 2013). In this case, the tool could support the organization spanning work of macroergonomics as described by Kleiner (2004), the integration of HF as identified by Dul and Neumann (2009), and the development of the HF discipline (Dul, et al., 2012; Norros, 2014). The conversations within the organization can also help HF specialists to better appreciate how their knowledge is, and can be, integrated in other domains in the organization, such as Village, et al. (2013a) found with the use of cognitive mapping. This appreciation of HF in the organization allowed the HF group in our case study to better focus future program changes, education outreach and prioritize resources accordingly. Further, the associated challenge to increase the HF score, along with the nonprescriptive, guiding criteria to move to a higher score, creates a target goal for the organization. The use of goal setting has been shown to affect performance across a range of environments with the setting of specific goals potentially providing improved performance (Latham and Yukl, 1975; Doran, 1981; Latham and Locke, 2007). Hierarchically arranging goals, as the maturity levels within the HF elements and the HF elements themselves create for the function, creates a short term feedback on progress while simultaneously extending the user's vision to the future (Zimmerman, 2008).

The tool development by consensus approach has been demonstrated in the development of standards, performance management systems, and organizational assessments. For example, the 
International Organization for Standardization (ISO) (International Organization for Standardization, 2013) and Canadian Standards Association (CSA) (Canadian Standards Association Group, 2015) utilize a panel of subject matter experts to develop their standards. Similarly, Kaplan and Norton (1996) and the European Foundation for Quality Management (2015) are examples of a management system and organizational assessment, respectively, that convened panels business experts for development.

Field testing of this tool facilitated development of a stakeholder approved and usable approach while simultaneously developing understanding of the potential use of the tool in the field. Style suggestions for the tool mostly focused on language clarity, managing the volume of information, and improving ease of data entry. For example, definitions were added alongside each of the HF elements in the Excel version to prevent page switching, while the addition of comment boxes for each element in a function allowed the user to provide context to the scoring and document key information, such as HF practices in place, for future comparison. In its current form, the tool would appear to score well with respect to applicable key HF tool factors (e.g. time to use, cost, training needs, difficulty to use, flexibility, usefulness relative to needs, and report usefulness) highlighted by Perez and Neumann (2015) in their examination of virtual HF tools. Further testing of the predictive validity of the tool with respect to organizational HF and performance outcomes, meaning the ability for the scores to predict future organization performance (Trochim and Donnelly, 2007), remains a task for future research and will be important to help support future implementation and use of the tool in organization processes.

\subsubsection{Limitations}

While those functions tested had positive results, the size of the document, volume of information, and time for the workshops limited a complete testing of content validity and HF maturity. The testing of the functions in the case field trial was conducted at the discretion of the industry partner. Resource constraints, organization restructuring and personal choice resulted in the testing of five functions. The current tool may show a manufacturing bias to HF due to the industry partner focus and common experience of some of the research team. Caution may need to be exerted when applying the tool at face value in other industries. Adding comment sections for each element potentially mitigates some of the concern when applying the tool outside of manufacturing as the user is able to describe the context in which they interpreted the individual 
element. The added consideration in the tool for knowledge employees alongside front line workers, a company suggested revision, further improves transferability of the tool and also demonstrates how the framework can extend to meet other needs, such as the inclusion of an IT function or further adaptation for organizations in other sectors. Regardless of the preceding tool additions, broader testing of the tool is needed with more users and organizations. Currently, the use of the tool in the field was primarily from the perspective of one person, in a case organization that was supported by one or two subject matter experts in the field. Without broader testing it is not possible to provide comments on both transferability to other organization sectors (e.g. service) or on the inter-rater reliability of the current format of the tool.

\subsection{Conclusion}

This chapter has presented the development and preliminary testing of a novel tool to assess the human factors (HF) capability and integration across an organization, an ability that has not been available with other HF tools. The creation of the Human Factors Integration Tool (HFIT) allows the user to score the integration and capability of HF in a number of supporting HF elements across five levels of maturity for up to 16 functions within an organization. Consensusbased development combined research and industry experience to develop and field test the tool while subject matter expert workshops contributed to face and content validity testing. The partnered design approach identified opportunities to improve tool content and usability which were implemented and reviewed. Industry participants expressed a need for this type of assessment tool and an appreciation and interest in what was developed. Workshop participants had a statistically significant non-neutral, supporting level of agreement with statements on the need for the tool and appropriateness of contents, definitions and reporting format. Scoring HF in the partner organization allowed HF specialists to better appreciate the level of understanding of HF in the organization as well as the effectiveness of HF programs. The ability of the tool to develop meaningful discussions with other organizational stakeholders about the contribution of HF in organizational performance simultaneous to employee well-being was a highlighted benefit of the use of the tool. Specialists saw the tool as supporting HF-related development projects that could be guided, with progress scored, by the tool. The tool is ready for broader validity testing across different organizations. 


\section{Chapter 8}

\section{Discussion, future work, and conclusion}

\section{Discussion and future work}

\subsection{Case and methods approach}

This dissertation presents views on metrics within the case organization and considers issues for HF metrics development (Chapter 3). The development of two HF tools demonstrates methods for assessing HF at the level of the workstation (Chapter 4) and the organization (Chapter 7). These also demonstrate approaches for developing new tools collaboratively with the user and their organization (Chapters 6 and 7). The limitation of this dissertation research is that it is from the case perspective. Case studies have been seen as limited in their study rigor and the ability to generate generalizable research (Yin, 2009). However, because of their specific focus, case studies have been lauded for their more in-depth investigations of single, complex phenomena (Yin, 2009). Though the findings from this research can be applied elsewhere in some instances and used to initiate further inquiry, future work is needed to confirm if similar results can be expected from other manufacturing organizations or completely different organizations such as health care.

As a whole, this dissertation used mixed methods research. The type of research method and data gathering depended on the approach necessary to understand the research questions. Though qualitative data was most common to address the research questions, quantitative results, such as in the testing of the workstation efficiency evaluator tool (Chapter 5) were more appropriate in some cases. The overarching action research (AR) approach in this dissertation, is used infrequently in HF research (Neumann, et al., 2012). AR has been promoted as means to generate better understanding of the implications of HF interventions (Rosecrance and Cook, 2000; Neumann, et al., 2012; Neumann and Village, 2012). The use of an AR based approach is advantageous in that it embeds the researcher within the change process to generate 
understanding from within as opposed to only observing from the outside (McTaggart, 1991; Ottosson, 2003). However, AR has been dismissed as soft science due to its lack of methodological rigour compared to hypothesis testing (Pasmore, 2001; Neumann, et al., 2012). Using AR in this dissertation provided an effective means to understand the functions of an organization and the implications of HF from a perspective within the organization. It complemented the user-centred development of tools and approaches and allowed the researchers to seek out collaborators while adjusting to an organization tasked with near continual restructuring for most of the project. Hypothesis based testing or case-control research would not have been able to adapt as well to the changes because of the rigid structure of those methods. The less rigid, more organic approach to exploratory research that AR affords could raise concern for results repeatability as the research evolved unscripted.

\subsection{Addressing the three objectives}

This dissertation strove to improve the understanding of HF metrics within a production organization by targeting three objectives. Each objective is discussed specifically in the following sections.

Objective 1: Determine the company stakeholder views of HF metrics, metrics development and HF application

The first objective in the study, presented in Chapter 3, found that engineers were more familiar with metrics in the case organization compared to HF specialists. The HF specialists had a health focus to their work and measurements that was consistent with Canadian HF specialist and ergonomist work (Theberge and Neumann, 2013). The relationship of HF metrics to the organization demonstrated the existence of HF in the 'ergonomics sidecar', meaning HF is a personnel related health concern of human resources (HR) and health and safety (H\&S), and HF being disconnected with design decisions (Jensen, 2002; Neumann, et al., 2009). Updating the framework illustrated in Figure 1, the weighting of the arrows representing HF metrics have been adjusted to reflect a greater number of reactive metrics compared to the non-existent predictive HF metrics (Figure 22). 


\section{Human Factors Metrics}

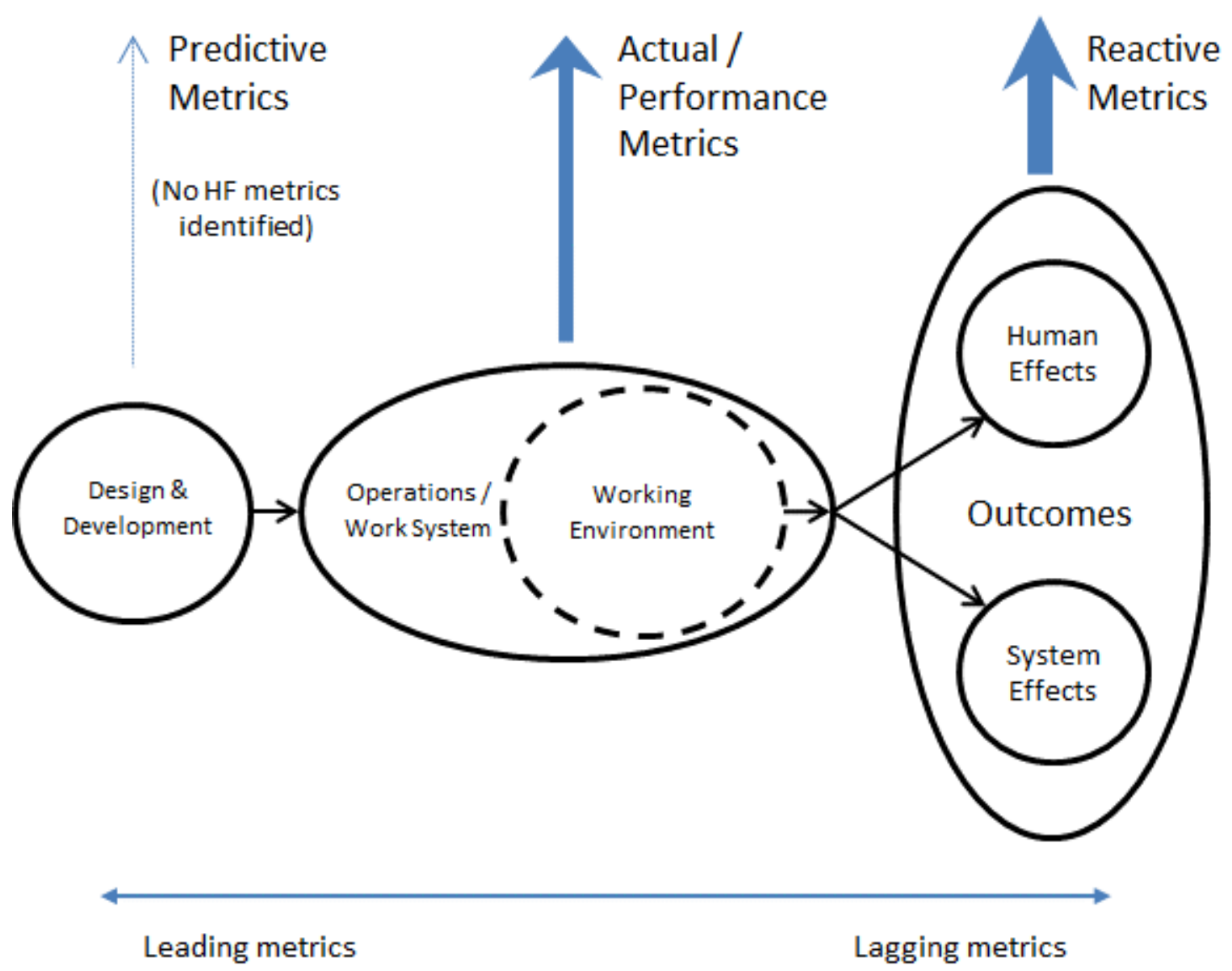

Figure 22. Updated framework from Figure 1 to illustrate the number of HF metrics observed in the case organization. Thicker arrows represent more metrics observed. Note that no predictive HF metrics were initially identified.

The HF focus on the human and system interaction in their environment means the potential exists for HF to move beyond HR and H\&S and span organizational boundaries (Dul, et al., 2012). Moving HF throughout an organization and away from an organization mindset of HF providing a health only benefit is substantial organizational change. Organizational change is a lengthy process with a low rate of success (Smith, 2003). Similarly, organizational initiatives like performance measurement system implementation are not always successful either (McCunn, 1998; Bourne et al., 1999; Hudson, et al., 2001).

Part of making the shift of HF in the organization is providing value to the new audience, an audience that may not be familiar with the contributions of HF beyond H\&S. For example, 
improving the integration of HF metrics into the design of the production systems in the case organization would require addressing gaps between engineering and HF specialists. Engineers were not fully aware of the contributions of HF to worker performance and work quality and HF specialists in the organization did not have the tool capability to generate metrics that related to the leading, status, lagging, and response themes of engineering metrics that were observed.

The lack of HF measures and indicators as a whole demonstrated a lack of metrics to create metrics sets and an HF metrics system. A lack of HF metrics limits the ability of HF information to contribute to engineering and management processes. Performance measurement systems focus on financial information, customer satisfaction, among others, but show little evidence for HF beyond employee engagement or typical H\&S metrics such as lost time injuries. In a management through measurement mindset, the contributions of HF can get left behind if the HF measures are disconnected. Neely, et al. (1995) have commented on the need for performance measures to be "positioned in a strategic context" since the result of measurement is more than quantification - it stimulates action. Connection to the organization and supporting the organization were two factors important for metric development observed in this case study. Understanding whether the absence of HF in performance measurement is due to ingrained thinking of managers and directors, a lack of awareness of the HF contribution, or lack of HF metrics could also be an avenue of future research to explore. Steps were taken to improve HF metrics through development of HF assessment and prediction capabilities in the organization, including adapting engineering tools (see Village, et al., 2014b), developing a workstation assessment tool (Chapter 4), and developing an organization assessment tool (Chapter 7). The organization assessment tool aside, these tools provided information predominantly for production system design in the case organization. More work is needed in the organization though to develop tools that provide information that contributes to product design and the associated performance metrics. Research in other organizations is also needed to better understand the types of organization critical metrics to which the HF discipline needs to contribute if HF tools are to be used by engineering and management teams. Further, research is needed to determine if the factors key to developing metrics are unique to the case organization or transferable to other organizations as well. It is also worth considering in future research the context of HF information required to improve HF integration in other engineering functions, or beyond engineering into business and strategy focussed functions within an organization. With 
improved understanding of HF metrics needs in this case and other organizations, the methods and tools to create a base of HF metrics can be established. These base HF metrics can be potentially used to create HF metrics sets and systems. Research can then be pursued to develop understanding of the needs for HF metric sets and systems and the integration of the sets and systems throughout an organization which can then help to get a better understanding of how HF contributes to performance and quality.

Objective 2: Develop a workstation level HF assessment tool for light assembly work

The workstation efficiency evaluator (WEE) tool developed and tested as described in Chapters 4 to 6 , addressed the need in the case organization to assess the effect of light assembly workstation design on the worker and work system. Other assessment tools that were available to the HF specialists in the organization (e.g. RULA (McAtamney and Corlett, 1993), REBA (Hignett and McAtamney, 2000), ACGIH HAL-TLV (American Congress of Governmental Industrial Hygienists, 2001; Marras and Karwowski, 2006)) were observation based, requiring a work system or workstation to be present to use the tools. These tools were not design oriented and did not allow for an assessment of health risks of light assembly workstation at the design stage, a gap noted by one HF specialist. The WEE tool addressed this gap and incorporated time prediction capabilities to improve its relevance to design decisions and estimating system time requirements. Though virtual HF tools were also commercially available to the organization, those tools are aimed primarily at manual material handling and heavy manufacturing, are more expensive and require specialized users (Lockett, et al., 2005; Lämkull et al., 2009; Perez and Neumann, 2015).

Important to the WEE tool design was the input from users and managers targeting specific information and a style of information to assist their operations (Chapter 6). These discussions contributed to the type of information collected and guided the results presentation format. Achieving this goal necessitated a participatory ergonomics or user-centred design approach, both known to improve design and adoption of new products and processes (Haims and Caryon, 1998; Mannonen and Nieminen, 2006). The range of stakeholders involved in the tool development in the case organization demonstrated top down and bottom up approaches to tool development. In the top down approach managers, for example, guided the focus of the tool, whereas in the bottom up approach the usability of the tool was more of a focus. The inclusion 
of engineers, managers and directors into the review and development of the tool highlighted how presentation of HF information needs to be better considered in HF tool development. For example, raw data should be summarized with the three most important issues highlighted at the top of the output information summary. More research is needed to better understand the HF connection to other organization interests since the context of HF information needs to contribute beyond health and safety concerns and address the dual HF goals of performance and well-being. Productivity and quality were important considerations by managers and directors for HF tool development (Village, et al., 2014b). Health information was also important to the managers and directors as a complement to the system information, highlighting the business and health alignment need suggested by Dul and Neumann (2009). This may mean that future HF tools need to have less focus on reporting about tissue loads, which do not relate as well, nor is the importance understood as well, to those without an extensive HF background. As an example in the WEE tool, the use of a simplified net shoulder moment prediction model may seem less valid to biomechanists. A truly realistic shoulder model would provide information on all principal shoulder movement axes. Outside of the comparison of two design options (A to B comparison), however, three-dimensional shoulder information has little context for most people. In part this is due to the complexity of the shoulder joint, the lack of appropriate load and moment threshold values and limited training of decision makers. Until more research is completed to fill this gap this simplified tool provides a "good enough" function that provides information to support conversations on implications of design choices.

Too often the researcher's focus on scientific precision and validity in a practitioner tool inhibits them from achieving a functional, usable 'good enough' tool which some practitioner's desire. Buckle (2011) has commented that the 'perfect is the enemy of the good' as it pertains to ergonomics research, where researcher goals and publishing requirements are disconnected from practitioner needs. A similar search for perfection of measures has been said about the development of metrics. Melnyk, et al. (2004) have noted that managers searching for quick, useful information are often satisfied with a measure that is 'good enough'. With this mindset, managers are demonstrating their satisfaction with measures that are sufficient to guide decisions, even when a given measure is not optimal, which is a decision process referred to as 'satisficing' (Simon, 1956; Simon, 1997). Applying this logic to tool development leads the developer towards 'satisficing' the tool requirements, meaning creating something satisfactory 
instead of optimized (Simon, 1956; Simon, 1997). With this in mind, further research should perhaps examine the 'good enough' threshold for tools. It is possible that there is precisionutility trade-off, where the threshold is related to the accuracy of the output information the audience requires.

With respect to the WEE tool, future work should investigate its use in more extensive field trials. A better understanding of factors contributing to the tool usability can be obtained from a more directed study of its use in other organizations. Future development of the tool capabilities could include the integration of a more mechanically complex shoulder load prediction model that also included a greater range of hand loads, with the caveat that a simultaneous investigation of the effective presentation of the outputs is needed. Other musculoskeletal risk factors could be included in the tool, such as gaze and head angles and improved fatigue prediction. Further, with the increased adoption of sit/stand workstations the tool could be adapted to work in these scenarios and tested for utility. To improve the tool relevance to system output, a comparison between predicted time and actual time in light assembly could be completed to provide commentary on the tool time prediction accuracy. Lastly, an investigation into the addition of human error prediction as an outcome, with similar logic as Givi et al. (2015) have used for dualresource constrained system modelling, could further strengthen the tool contributions to system outcomes.

Objective 3: Create a tool that reports the level of HF integration and maturity in an organization

Chapter 7 described the creation and testing of an approach to assess HF at the organization level. The application of the tool allows the tool user to gain an appreciation of the level of HF integration in the organization. This type of approach has not yet been available.

Testing in the case organization and in workshops demonstrated a desire from participants for this type of tool. The tool facilitates thinking of HF throughout the organization beyond the more common HF involvement at the workstation level. The macro-focussed tool thus contributes to moving HF thinking from the micro, workstation level, to the macro, organization level. When HF is thought of at a macro level, as with macroergonomics, HF is seen in its organizational context (Hendrick and Kleiner, 2001; Kleiner, 2006). 
User suggested modifications to the tool ensured that the assessment captured more than health benefits to a frontline worker. The assessments of knowledge worker activities and the capability of worker improvement along with physical and psychosocial risk factors were added based on the interactions and expressed needs of tool users. These modifications develop the potential for the tool to provide a more complete macroergonomic assessment and potentially look at the HF contributions an organization creates beyond the organizational boundaries.

Future research with the tool could investigate how well an organization's score relates to the employee satisfaction at work and outside of work. Perhaps more pressing research for the tool is to determine how outcome scores relate to organizational performance and how the approach developed in the case organization transfers to organizations with a different focus. Direct testing in other organizations is required to determine tool transferability to organizations beyond the case. Workshop participants, who were not members of the case organization, expressed an interest for the tool and commented on its relevance to their particular organizations. Changes implemented from workshop feedback means the transferability of the tool is better than if the tool had only been reviewed with the case organization. One last point for research to consider in the short term is an investigation into the importance of the different HF elements to organizational performance. Knowing more of how individual HF elements directly contribute to organization and worker performance would have two substantial benefits to future versions of the tool. The knowledge would provide guidance with respect to weighting of the scoring and which elements to target first in HF continuous improvement initiatives.

\subsection{General discussion and future work}

The findings of this exploratory case research provide a basis for further investigation and reflection on HF measurement and reporting in organizations. Combined, the results demonstrated that the full impact of HF is still not well known and that HF metrics do not merge well with organization metrics in their current form. Common though, in all of the results, is that HF metrics, measures, and reporting needs HF approaches in their development. These approaches include participative ergonomics, user involvement, and user-centered design, where the requirements and interests of users and target audiences are considered during development. Participative approaches, such as participative ergonomics and user-centred design, have been shown to benefit development and uptake of organizational process (Haims and Caryon, 1998; 
Mannonen and Nieminen, 2006). These approaches are not without challenges due to techniques used, personnel involved, or resource constraints (Eason, 1995; Mankin et al., 1997; Garrety and Badham, 1999; Cole et al., 2009; Driessen, et al., 2010; van Eerd, et al., 2010). In this dissertation, a project manager developing new performance metrics found a range of different ideas related to the specific interests of stakeholders. Garrety and Badham (1999) have noted something similar in technology development. Development challenges in their case examination were not due to HF methodologies but were a function of individual stakeholder power and personal interest and the political process necessary for sociotechnical change. Even with these identified challenges, new HF tools should ideally be developed in collaboration with potential users so that researchers can better understand the design needs. This includes understanding the necessity of precision and accuracy in the reporting of tool outputs that is needed for making business decisions.

The collaborative development of the two tools in this dissertation demonstrated a researcherpractitioner partnership, addressing gaps in measurement and reporting but also allowed the investigation of design requirements from the user's perspective. Even with this information, more work is needed to better understand the requirements of effective HF tool design across a broader audience of organizations. Feedback from engineers and practitioners in this dissertation assisted with creating more relevant tools and hopefully increased the potential for tool adoption. The benefits of user-centered design to improve product and process design are well documented (for example, Kujala, 2003; Abras et al., 2004). Little research has been done though into the process of designing HF tools "in vivo". This work has highlighted the need for HF reporting to better combine performance and well-being in tool design. Previous HF tools have not effectively addressed this issue and instead often focused only on reporting on musculoskeletal disorder risk factors. This was evident in the case organization as the HF specialists did not have the ability to provide other forms of HF information beyond a commentary on health risk. It was not that the information being reported was the wrong information, there was no other option available to present another type of HF information. The HF specialists were left with piecemeal measures to assess workstation designs and waiting until work structures were in place to review the layout and apply the existing HF guidelines. Though the available tools were valid, they limited the application of HF and the capability to move HF beyond health and safety concerns and into design decisions. 
Tools are needed to better connect HF to the organization without losing employee well-being as a consideration. These types of HF tools and measures are not a change of thinking since HF is promoted as benefiting performance and well-being (Dul, et al., 2012; International Ergonomics Association, 2014). New research needs to include light assembly tool development that includes prediction for well-being and performance outcomes in a format that is affordable for all practitioners. More work is also needed to improve the assessment of HF capability across the organization. Few in the case organization understood the performance benefits of HF consideration in assembly operations and even fewer participants understood the implications of HF to organization functions like human resources, finance or sales. Practitioner development of new tools is possible though development success may be dependent on time available. The focus on day-to-day operations that deliver value to the customer makes tool development by practitioners in the field difficult. Tool creation should also not be left solely to researchers. As evident by the tools that exist, the focus of research-based tools are primarily on health risk factors and have little connection to employee performance. Further, some tools have great precision, biological relevance, and are no doubt valid and reliable, but lack a context for non-HF specialists. Collaborative tool creation by researchers and practitioners would appear to be ideal. This arrangement is not easy. More work is required to understand how best to foster these interactions for tool development.

Tool designers are not solely to blame though on their lack of performance reporting measures. HF research is increasingly demonstrating the connection between quality and other measures of performance (for example: Helander and Burri, 1995; Drury, 2000; Meerding, et al., 2005; Yeow and Sen, 2006; Erdinc and Vayvay, 2008b; Falck, et al., 2010). Connecting design decisions to both HF risk and worker performance in the short or long term is still not well developed. Even with the increasing amount of evidence of the connection between HF and system performance, research is needed to better understand the direct impact on worker performance from poor HF application (i.e. a worker health risk ' $X$ ' leads to quality deficit of magnitude ' $Y$ '). As found in the case experience, the precision and detail of these connections need not be perfect but should provide a means of generating discussions on the impact of design decisions on worker performance and well-being. The ability to better quantify the financial impact of HF will be greatly improved once the HF-performance relationship is better understood. This improvement opens the door to increased interest in HF and, ultimately, more HF integration throughout 
organizations. With improved HF integration, employee performance and well-being should increase.

This dissertation highlighted the need for more work to improve basic HF measures in order to generate HF metrics. Moving beyond HF metrics to integrating within the different aspects of an organization provides room for further exploration. Researchers have called for HF to be a part of strategy decisions (for example: Jensen, 2002; Dul and Neumann, 2009; Dul, et al., 2012), while others have promoted the value of stakeholders in strategic decisions (for example: Freeman, 1994; Freeman et al., 2004). Involving many stakeholders in strategic decisions highlights the importance of actor network theory, where a complex network of actors all contribute to organisational change efforts (Kaghan and Bowker, 2001).

HF integrated as a part of strategy begs the question of how HF metrics would integrate in the reporting structure at the strategic level of an organization. HF currently has more reporting capabilities at the workstation level. More leading, design metrics necessitate a move up the organization structure, with strategy metrics even further up from the workstation level. How HF measures fit and interact at the different levels within this hierarchy requires further research.

Organizational scorecards provide an overview of subordinate groups, as do metric sets (Melnyk, et al., 2004). Conceivably scorecards of higher levels are supported by lower level scorecards (Huwe, 2010). The tiered formation funnels aggregated lower level information up the hierarchy of the organization. Aligning metrics in organizations from top to bottom is challenging because of the noise created and the aggregation required of metrics (Melnyk, et al., 2005). Conceivably HF information would need to be similarly aggregated which requires further research to how this is done effectively. HF reporting and impact on higher level scorecards will be challenged even if there is an understanding of HF information aggregation. One point for investigation is how HF competes for scorecard space at higher levels, since 15-20 metrics are considered near the threshold for a person to manage (Brown, 2007). A second point is that if HF is a means to an end then will HF impact be built within other reporting measures, leaving it a non-measured, non-managed contribution at the upper organization levels. This system aspect of HF metrics warrants further research. 


\subsection{Contributions}

The findings from this dissertation contribute to the industry partner, practitioners and the research community. The contributions from each objective and the dissertation as a whole are as follows:

Objective 1: Stakeholder views of HF metrics, metrics development and HF application.

- This dissertation has improved the understanding of HF metrics in the case organization with suggestions for future metrics and tool development.

- HF metrics in the case organization have been identified as reactive, health supporting information that is of a different focus compared to the four identified engineering metric categories of leading, status, lagging, and response.

- Evidence has been provided in the case organization that HF and engineering metrics are disconnected, in part due to a lack of predictive tools, an HF focus on health outcomes, and operation silos that reinforce HF stereotypes.

- Five important issues have been identified for developing HF metrics - knowledge, connection, support, resources, and communication.

- Practical advice has been created for HF practitioners interested in developing HF metrics.

Objective 2: Developing a workstation level HF assessment tool for light assembly work.

- A novel virtual HF tool for light assembly workstation assessment was created that improves assessment capabilities of HF practitioners and engineers. The tool enables the simultaneous assessment of worker risk and system outputs, which is uncommon in most HF tools.

- Testing of the tool showed that there is a low impact of measurement input error on tool outputs of movement time and shoulder load.

- The application of the tool allows layout prediction and workflow planning from light assembly worker and system information, which can be completed at the design stage using design information. The tool is an improvement over existing observational methods, as was demonstrated using a Swedish electronics assembly system case example found in the scientific literature. 
- It was demonstrated within this dissertation how a user-centered design approach to HF tool development can improve the design of the tool and the relevance and display of output information.

Objective 3: Creating a tool reporting the level of HF integration and maturity in an organization.

- A novel macroergonomic assessment tool has been developed that has demonstrated good face and content validity. The tool, which was expressed as needed by subject matter experts, provides an organization level assessment capability of HF integration and maturity that has been previously unavailable for practitioners, especially when assessing HF integration beyond health and safety.

- The tool provides a mechanism to facilitate dialogue about the impact of HF on organization performance and worker well-being throughout an organization, as evident from the process of trialing the tool in the case organization. This dialogue furthered the awareness of HF and educated people that were unfamiliar with HF, while simultaneously providing a venue to capture and score the integration and maturity of HF in different functions of the organization.

- The workshops to assess validity provided exposure to the tool beyond the case organization which improved the transferability of the tool to other organizations.

- The development process of the tool provided a demonstration of how a consensus and participative approach to tool design helps to develop a valid tool and enhance the relevance of the tool to future users.

Overarching contribution of the dissertation:

- Taken as a whole, this dissertation provides evidence that HF metrics and tools development need HF principles applied in their development in order to create HF information that is relevant and applicable to engineers and other organization decision makers. Further, this dissertation provides two examples, one from a micro HF perspective and the other from a macro HF perspective, of how to develop new metrics and tools with input from those who collect and use the information. 


\subsection{Conclusion}

This dissertation takes an exploratory look at HF metrics within a product and production design electronics organization. From this case investigation, a better understanding of the requirements for HF metric development and HF measurement and reporting needs in the organization was created. Engineers were more familiar with the idea of metrics than HF practitioners. HF metrics identified did not match with all of the established engineering metrics categories. No leading HF metrics existed for design and most HF metrics were lagging and health focused. In part, this was due to HF metrics focussing more on health and safety measures and activities being completed. The differences pointed to further gaps in the understanding of HF contributions and the need for new HF tools to generate reporting measures. Stakeholder interactions highlighted five key points to the development of HF metrics. These included knowledge, communication, resources, connection, and support. Two approaches to generating new HF metrics were developed and tested. A workstation efficiency evaluator tool was one tool created. It allowed assessment of seated light assembly workstations in the design stage and reported on employee health risk factors along with predicted performance time. The tool performed well in a comparison to an observation-based analysis and also demonstrated tolerance to input errors on workstation outcomes. Development of the tool was guided by a collaborative user-centered design approach which helped determine data of interest and effective communication of output variables. A second tool that was developed allowed for the assessment of the maturity of HF capability and integration in the organization. The consensusbased tool received positive review in the case organization and, as one of its benefits, provided a means for the human factors specialist to initiate dialogue about the contribution of HF within the organization. Field testing and workshop-based review tested face and content validity. Workshop participant ratings showed a statistically significant agreement with statements on the need for the tool, and the appropriateness of contents, definitions, and reporting format. The two developed tools created new means to measure and report HF and also demonstrated approaches to the development of future HF tools. As a whole, these dissertation findings illustrate the need for more HF metric work, especially the development of HF measures that can contribute to organizational outcomes. This dissertation also illustrates that any development of HF measures and processes need HF in the development, meaning a collaborative approach that designs for the user and encapsulates the dual HF goals of performance and well-being. 


\section{Appendices}

\section{Appendix A. Example of data input tables for WEE tool.}

Table A. Example of hand location and element MTM-1 classification input data to tool.

\begin{tabular}{clcccccc}
\hline & & \multicolumn{3}{c}{ Left Hand } & \multicolumn{3}{c}{ Right Hand } \\
Element \# & \multicolumn{1}{c}{ Description } & Right/Left & Fwd/Bkwd & Up/Down & Right/Left & Fwd/Bkwd Up/Down \\
\hline 1 & Handling Board/frame (get frame) & -40 & -20 & -21.7 & 20 & 17.2 & 0 \\
2 & Place frame & -20 & 17.2 & 0 & 20 & 17.2 & 0 \\
3 & Handling Board/frame (get board) & -40 & -20 & -21.7 & 20 & 17.2 & 0 \\
4 & Framing activity & -20 & 17.2 & 0 & 20 & 17.2 & 0 \\
5 & Reach component 1 & -40 & 8 & 4.5 & 20 & 17.2 & 0 \\
6 & Place component & -15 & 17.2 & 0 & -15 & 17.2 & 0 \\
7 & Reach component 2 & -37 & 18 & 4.5 & -15 & 17.2 & 0 \\
$:$ & & $:$ & $:$ & $:$ & $:$ & $:$ & $:$ \\
40 & Handling Board/frame (asiding) & 20 & 0 & 0 & 40 & -20 & -21.7 \\
41 & Transport (spin/move) & 20 & 0 & 0 & 40 & 11.8 & 7.8 \\
\hline
\end{tabular}

\begin{tabular}{|c|c|c|c|c|c|}
\hline \multirow[b]{2}{*}{ Element \# } & \multirow[b]{2}{*}{ Description } & \multicolumn{4}{|c|}{ Left Hand } \\
\hline & & 3D Distance & Reach/Move & Grasp or Release (R) & Class of Fit \\
\hline 1 & Handling Board/frame (get frame) & 0.00 & Reach & G - very small, flat on surface & (n/a) \\
\hline 2 & Place frame & 47.48 & Move & $\mathrm{R}$ - normal release & Close - Light Press. - NS-E \\
\hline 3 & Handling Board/frame (get board) & 47.48 & Reach & G - very small, flat on surface & $(\mathrm{n} / \mathrm{a})$ \\
\hline 4 & Framing activity & 47.48 & Move & $\mathrm{R}$ - normal release & Close - Light Press. - NS-E \\
\hline 5 & Reach component 1 & 22.47 & Reach & G - interference - diameter $>1 / 2 "$ & $(\mathrm{n} / \mathrm{a})$ \\
\hline 6 & Place component & 27.02 & Move & $\mathrm{R}$ - normal release & Exact - Heavy Press. - NS-E \\
\hline 7 & Reach component 2 & 22.47 & Reach & G - interference - diameter $>1 / 2 "$ & $(n / a)$ \\
\hline : & : & : & : & : & $:$ \\
\hline 40 & Handling Board/frame (asiding) & 43.54 & Move & $\mathrm{R}$ - normal release & Loose - No Press. - SS-E \\
\hline 41 & Transport (spin/move) & 0.00 & Reach & (n/a) & (n/a) \\
\hline
\end{tabular}




\section{Appendix B. Coefficients for models with different ranges.}

Data presented in Table B1 and B2 provide the coefficients for the following equations:

Net Shoulder Moment $(\mathrm{Nm})$

$$
\begin{aligned}
& =\text { Intercept }+X \text { Coefficient } \times x\left(\text { hand }_{(m)}-\text { shoulder }_{(m)}\right)+Y \text { Coefficient } \\
& \times y\left(\operatorname{hand}_{(m)}-\operatorname{shoulder}_{(m)}\right)
\end{aligned}
$$

Net $\% M V C(\%)$

$$
\begin{aligned}
& =\left(\text { Intercept }+X \text { Coefficient } \times x\left(\text { hand }_{(m)}-\text { shoulder }_{(m)}\right)+Y\right. \text { Coefficient } \\
& \left.\times y\left(\operatorname{hand}_{(m)}-\operatorname{shoulder}_{(m)}\right)\right) * 100 \%
\end{aligned}
$$

Where: $x\left(\right.$ hand $_{(\mathrm{m})}$-shoulder $\left.(\mathrm{m})\right)$ is the forward/backward hand location with respect to the shoulder joint and $y\left(\right.$ hand $_{(\mathrm{m})}$-shoulder $\left.(\mathrm{m})\right)$ is the hand location distance from the shoulder in the up/down direction.

Table B1. Set of equations and $\mathrm{R}^{2}$ values for Full Range developed shoulder model. Where, $\mathrm{X}$ and $\mathrm{Y}$ Coefficients are for horizontal and vertical components of the equation, respectively.

\begin{tabular}{cccccc}
\hline & Data Set & $\mathrm{R}^{2}$ & Intercept & X Coefficient & Y Coefficient \\
\hline & 5\%ile Female & 0.84 & 0.321 & 8.437 & 1.709 \\
& 50\%ile Female & 0.84 & 0.426 & 10.702 & 2.117 \\
Net Shoulder & 95\%ile Female & 0.84 & 0.678 & 14.608 & 2.886 \\
Moment (Nm) & 5\%ile Male & 0.85 & 0.416 & 10.883 & 2.053 \\
& 50\%ile Male & 0.85 & 0.590 & 14.489 & 2.724 \\
& 95\%ile Male & 0.86 & 0.858 & 19.167 & 3.592 \\
\hline 5\%ile Female & 0.81 & 0.009 & 0.288 & 0.080 \\
Net \%MVC & 50\%ile Female & 0.82 & 0.012 & 0.365 & 0.099 \\
(\%) & 95\%ile Female & 0.81 & 0.021 & 0.494 & 0.136 \\
& 5\%ile Male & 0.81 & 0.006 & 0.180 & 0.053 \\
& 50\%ile Male & 0.81 & 0.009 & 0.239 & 0.071 \\
& 95\%ile Male & 0.82 & 0.013 & 0.316 & 0.094 \\
\hline
\end{tabular}


Table B2. Set of equations and $\mathrm{R}^{2}$ values for Below Shoulder developed shoulder model. Where, $\mathrm{X}$ and $\mathrm{Y}$ Coefficients are for the horizontal and vertical components of the equation, respectively.

\begin{tabular}{cccccc}
\hline & Data Set & $\mathrm{R}^{2}$ & Intercept & X Coefficient & Y Coefficient \\
\hline & 5\%ile Female & 0.91 & -1.658 & 11.717 & -1.691 \\
& 50\%ile Female & 0.91 & -2.310 & 14.715 & -2.060 \\
Net Shoulder & 95\%ile Female & 0.91 & -3.525 & 20.492 & -2.960 \\
Moment (Nm) & 5\%ile Male & 0.92 & -2.184 & 14.726 & -1.925 \\
& 50\%ile Male & 0.92 & -3.120 & 19.573 & -2.645 \\
& 95\%ile Male & 0.92 & -4.477 & 25.861 & -3.470 \\
\hline 5\%ile Female & 0.85 & -0.059 & 0.395 & -0.047 \\
Net \%MVC & 50\%ile Female & 0.85 & -0.083 & 0.496 & -0.058 \\
95) & 95ile Female & 0.85 & -0.127 & 0.691 & -0.083 \\
& 5\%ile Male & 0.84 & -0.035 & 0.234 & -0.019 \\
& 50\%ile Male & 0.84 & -0.050 & 0.310 & -0.025 \\
& 95\%ile Male & 0.84 & -0.071 & 0.410 & -0.033 \\
\hline
\end{tabular}




\section{Appendix C. $\mathrm{R}^{2}$ values and equation coefficients for additional models created.}

Data presented in Table C1 provide the coefficients for the following equations:

Load Specific Models - for a given hand load

Net Shoulder Moment $(\mathrm{Nm})$

$$
\begin{aligned}
& =\text { Intercept }+X \text { Coefficient } \times x\left(\text { hand }_{(m)}-\text { shoulder }_{(m)}\right)+Y \text { Coefficient } \\
& \times y\left(\operatorname{hand}_{(m)}-\operatorname{shoulder}_{(m)}\right)
\end{aligned}
$$

Net $\% M V C(\%)$

$$
\begin{aligned}
& =\left(\text { Intercept }+X \text { Coefficient } \times x\left(\text { hand }_{(m)}-\text { shoulder }_{(m)}\right)+Y\right. \text { Coefficient } \\
& \left.\times y\left(\operatorname{hand}_{(m)}-\operatorname{shoulder}_{(m)}\right)\right) * 100 \%
\end{aligned}
$$

General Load Models - Hand load as an input

Net Shoulder Moment (Nm)

$$
\begin{aligned}
& =\text { Intercept }+X \text { Coefficient } \times x\left(\text { hand }_{(m)}-\text { shoulder }_{(m)}\right)+Y \text { Coefficient } \\
& \times y\left(\operatorname{hand}_{(m)}-\text { shoulder }_{(m)}\right)+\text { Load Coefficient } \times\left(\operatorname{hand~load}_{(g)}\right)
\end{aligned}
$$

Net $\% M V C(\%)$

$$
\begin{aligned}
& =\left(\text { Intercept }+X \text { Coefficient } \times x\left(\text { hand }_{(m)}-\text { shoulder }_{(m)}\right)+Y\right. \text { Coefficient } \\
& \left.\times y\left(\operatorname{hand}_{(m)}-\operatorname{shoulder}_{(m)}\right)+\text { Load Coefficient } \times\left(\text { hand load }_{(g)}\right)\right) \times 100 \%
\end{aligned}
$$

Where: $x$ (hand-shoulder) is the forward/backward hand location (m) with respect to the shoulder joint, $y$ (hand-shoulder) is the hand location distance (m) from the shoulder in the up/down direction and hand load is the hand load (g) lifted. 
Table C1. Set of equations and $\mathrm{R}^{2}$ values for $25 \mathrm{~g}$ hand load specific shoulder models. Where, $\mathrm{X}$ and $\mathrm{Y}$ Coefficients are for horizontal and vertical components of the equation, respectively.

\begin{tabular}{cccccc}
\hline & Data Set & & & $\begin{array}{c}\text { X } \\
\text { Coefficient }\end{array}$ & $\begin{array}{c}\text { Y } \\
\text { Coefficient }\end{array}$ \\
\hline & 5\%ile Female & 0.86 & 0.656 & 8.109 & 2.380 \\
Net & 50\%ile Female & 0.86 & 0.871 & 10.188 & 2.925 \\
Shoulder & 95\%ile Female & 0.86 & 1.295 & 13.857 & 3.887 \\
Moment & 5\%ile Male & 0.87 & 0.855 & 10.395 & 2.811 \\
$(\mathrm{Nm})$ & 50\%ile Male & 0.88 & 1.169 & 13.820 & 3.755 \\
& 95\%ile Male & 0.88 & 1.659 & 18.329 & 4.964 \\
\hline & 5\%ile Female & 0.83 & 0.019 & 0.276 & 0.099 \\
Net & 50\%ile Female & 0.83 & 0.027 & 0.344 & 0.124 \\
$(\%)$ & 95\%ile Female & 0.81 & 0.042 & 0.464 & 0.166 \\
& 5\%ile Male & 0.81 & 0.013 & 0.169 & 0.064 \\
& 50\%ile Male & 0.82 & 0.019 & 0.224 & 0.085 \\
& 95\%ile Male & 0.82 & 0.027 & 0.295 & 0.112 \\
\hline
\end{tabular}

Table C2. Set of equations and $\mathrm{R}^{2}$ values for $50 \mathrm{~g}$ hand load specific shoulder models. Where, $\mathrm{X}$ and $\mathrm{Y}$ Coefficients are for horizontal and vertical components of the equation, respectively.

\begin{tabular}{cccccc}
\hline & Data Set & & & $\mathrm{X}$ & Y \\
& 5\%ile Female & 0.87 & 0.609 & 8.452 & 2.356 \\
Coefficient & Coefficient \\
Net & 50\%ile Female & 0.87 & 0.868 & 10.398 & 2.946 \\
Moulder & 95\%ile Female & 0.86 & 1.235 & 14.214 & 3.881 \\
$(\mathrm{Nm})$ & 5\%ile Male & 0.88 & 0.803 & 10.757 & 2.784 \\
& 50\%ile Male & 0.88 & 1.188 & 14.070 & 3.732 \\
& 95\%ile Male & 0.88 & 1.696 & 18.495 & 4.961 \\
\hline 5et & 5\%ile Female & 0.83 & 0.019 & 0.284 & 0.100 \\
\%VC & 50\%ile Female & 0.83 & 0.027 & 0.353 & 0.125 \\
& 95\%ile Female & 0.81 & 0.042 & 0.472 & 0.167 \\
& 5\%ile Male & 0.81 & 0.013 & 0.173 & 0.064 \\
& 50\%ile Male & 0.82 & 0.019 & 0.228 & 0.085 \\
& 95\%ile Male & 0.82 & 0.027 & 0.299 & 0.113 \\
\hline
\end{tabular}


Table C3. Set of equations and $\mathrm{R}^{2}$ values for $100 \mathrm{~g}$ hand load specific shoulder models. Where, $\mathrm{X}$ and $\mathrm{Y}$ Coefficients are for horizontal and vertical components of the equation, respectively.

\begin{tabular}{cccccc}
\hline & Data Set & $\mathrm{R}^{2}$ & Intercept & $\begin{array}{c}\mathrm{X} \\
\text { Coefficient }\end{array}$ & $\begin{array}{c}\text { Y } \\
\text { Coefficient }\end{array}$ \\
\hline & 5\%ile Female & 0.89 & 0.590 & 9.022 & 2.319 \\
Net & 50\%ile Female & 0.88 & 0.858 & 10.937 & 2.914 \\
Shoulder & 95\%ile Female & 0.87 & 1.300 & 14.574 & 3.911 \\
Moment & 5\%ile Male & 0.89 & 0.878 & 11.053 & 2.823 \\
$(\mathrm{Nm})$ & 50\%ile Male & 0.89 & 1.152 & 14.605 & 3.744 \\
& 95\%ile Male & 0.88 & 1.673 & 19.002 & 4.923 \\
\hline & 5\%ile Female & 0.84 & 0.019 & 0.301 & 0.101 \\
Net & 50\%ile Female & 0.83 & 0.027 & 0.370 & 0.126 \\
$(\%)$ & 95\%ile Female & 0.82 & 0.042 & 0.489 & 0.168 \\
& 5\%ile Male & 0.82 & 0.013 & 0.181 & 0.065 \\
& 50\%ile Male & 0.82 & 0.018 & 0.237 & 0.086 \\
\hline
\end{tabular}

Table C4. Set of equations and $\mathrm{R}^{2}$ values for $200 \mathrm{~g}$ hand load specific shoulder models. Where, $\mathrm{X}$ and $\mathrm{Y}$ Coefficients are for horizontal and vertical components of the equation, respectively.

\begin{tabular}{cccccc}
\hline & Data Set & & & $\begin{array}{c}\mathrm{X} \\
\text { Coefficient }\end{array}$ & $\begin{array}{c}\text { Y } \\
\text { Coefficient }\end{array}$ \\
\hline & 5\%ile Female & 0.89 & 0.646 & 9.817 & 2.358 \\
Net & 50\%ile Female & 0.89 & 0.848 & 11.945 & 2.940 \\
Shoulder & 95\%ile Female & 0.88 & 1.263 & 15.665 & 3.836 \\
Moment & 5\%ile Male & 0.90 & 0.837 & 12.123 & 2.814 \\
$(\mathrm{Nm})$ & 50\%ile Male & 0.90 & 1.154 & 15.585 & 3.746 \\
& 95\%ile Male & 0.89 & 1.691 & 19.923 & 4.954 \\
\hline & 5\%ile Female & 0.85 & 0.018 & 0.335 & 0.103 \\
Net & 50\%ile Female & 0.84 & 0.026 & 0.404 & 0.128 \\
$(\%)$ & 95\%ile Female & 0.82 & 0.041 & 0.522 & 0.170 \\
& 5\%ile Male & 0.83 & 0.013 & 0.197 & 0.066 \\
& 50\%ile Male & 0.83 & 0.018 & 0.253 & 0.087 \\
\hline 95\%ile Male & 0.83 & 0.027 & 0.323 & 0.115 \\
\hline
\end{tabular}


Table C5. Set of equations and $\mathrm{R}^{2}$ values for $400 \mathrm{~g}$ hand load specific shoulder models. Where, $\mathrm{X}$ and $\mathrm{Y}$ Coefficients are for horizontal and vertical components of the equation, respectively.

\begin{tabular}{cccccc}
\hline & Data Set & $\mathrm{R}^{2}$ & Intercept & $\begin{array}{c}\mathrm{X} \\
\text { Coefficient }\end{array}$ & $\begin{array}{c}\text { Y } \\
\text { Coefficient }\end{array}$ \\
\hline & 5\%ile Female & 0.92 & 0.601 & 11.933 & 2.313 \\
Net & 50\%ile Female & 0.91 & 0.859 & 13.903 & 2.956 \\
Shoulder & 95\%ile Female & 0.89 & 1.285 & 17.501 & 3.881 \\
Moment & 5\%ile Male & 0.92 & 0.808 & 14.202 & 2.799 \\
$(\mathrm{Nm})$ & 50\%ile Male & 0.91 & 1.125 & 17.583 & 3.728 \\
& 95\%ile Male & 0.90 & 1.679 & 21.951 & 4.954 \\
\hline & 5\%ile Female & 0.86 & 0.017 & 0.403 & 0.108 \\
Net & 50\%ile Female & 0.86 & 0.024 & 0.472 & 0.133 \\
$(\%)$ & 95\%ile Female & 0.84 & 0.039 & 0.589 & 0.174 \\
& 5\%ile Male & 0.84 & 0.012 & 0.230 & 0.069 \\
& 50\%ile Male & 0.84 & 0.017 & 0.286 & 0.090 \\
\hline
\end{tabular}

Table A6. Set of equations and $\mathrm{R}^{2}$ values for $700 \mathrm{~g}$ hand load specific shoulder models. Where, $\mathrm{X}$ and $\mathrm{Y}$ Coefficients are for horizontal and vertical components of the equation, respectively.

\begin{tabular}{cccccc}
\hline & Data Set & & & $\begin{array}{c}\text { X } \\
\text { Coefficient }\end{array}$ & $\begin{array}{c}\text { Y } \\
\text { Coefficient }\end{array}$ \\
\hline & 5\%ile Female & 0.94 & 0.593 & 14.913 & 2.293 \\
Net & 50\%ile Female & 0.93 & 0.891 & 16.771 & 3.009 \\
Shoulder & 95\%ile Female & 0.91 & 1.266 & 20.447 & 3.789 \\
Moment & 5\%ile Male & 0.94 & 0.850 & 17.034 & 2.858 \\
$(\mathrm{Nm})$ & 50\%ile Male & 0.93 & 1.153 & 20.460 & 3.688 \\
& 95\%ile Male & 0.92 & 1.726 & 24.808 & 4.979 \\
\hline & 5\%ile Female & 0.88 & 0.015 & 0.505 & 0.115 \\
Net & 50\%ile Female & 0.87 & 0.022 & 0.573 & 0.140 \\
$(\%)$ & 95\%ile Female & 0.85 & 0.037 & 0.688 & 0.180 \\
& 5\%ile Male & 0.85 & 0.011 & 0.279 & 0.074 \\
& 50\%ile Male & 0.85 & 0.016 & 0.334 & 0.094 \\
& 95\%ile Male & 0.85 & 0.025 & 0.405 & 0.123 \\
\hline
\end{tabular}


Table C7. Set of equations and $\mathrm{R}^{2}$ values for $1000 \mathrm{~g}$ hand load specific shoulder models. Where, $\mathrm{X}$ and $\mathrm{Y}$ Coefficients are for horizontal and vertical components of the equation, respectively.

\begin{tabular}{cccccc}
\hline & Data Set & & & $\begin{array}{c}\text { X } \\
\text { Coefficient }\end{array}$ & $\begin{array}{c}\text { Y } \\
\text { Coefficient }\end{array}$ \\
\hline & 5\%ile Female & 0.96 & 0.654 & 17.649 & 2.348 \\
Net & 50\%ile Female & 0.95 & 0.878 & 19.715 & 2.911 \\
Shoulder & 95\%ile Female & 0.92 & 1.236 & 23.359 & 3.719 \\
Moment & 5\%ile Male & 0.95 & 0.802 & 20.073 & 2.820 \\
$($ Nm) & 50\%ile Male & 0.94 & 1.174 & 23.370 & 3.726 \\
& 95\%ile Male & 0.93 & 1.715 & 27.757 & 4.973 \\
\hline & 5\%ile Female & 0.89 & 0.013 & 0.607 & 0.122 \\
Net & 50\%ile Female & 0.89 & 0.020 & 0.675 & 0.147 \\
$(\%)$ & 95\%ile Female & 0.86 & 0.035 & 0.788 & 0.186 \\
& 5\%ile Male & 0.86 & 0.010 & 0.327 & 0.079 \\
& 50\%ile Male & 0.86 & 0.016 & 0.382 & 0.100 \\
& 95\%ile Male & 0.85 & 0.024 & 0.454 & 0.128 \\
\hline
\end{tabular}

Table C8. Set of equations and $\mathrm{R}^{2}$ values for general hand load shoulder models. Where, $\mathrm{X}$ and $\mathrm{Y}$ Coefficients are for horizontal and vertical components of the equation, respectively, and Load Coefficient is for the hand load being lifted.

\begin{tabular}{ccccccc}
\hline & Data Set & & & $\begin{array}{c}\text { X } \\
\text { Coefficient }\end{array}$ & $\begin{array}{c}\text { Y } \\
\text { Coefficient }\end{array}$ & Load Coefficient \\
\hline & 5\%ile Female & 0.90 & -0.330 & 10.972 & 2.341 & 0.00308 \\
Net & 50\%ile Female & 0.89 & -0.204 & 12.979 & 2.941 & 0.00346 \\
Shoulder & 95\%ile Female & 0.88 & 0.109 & 16.667 & 3.843 & 0.00374 \\
Moment & 5\%ile Male & 0.90 & -0.236 & 13.232 & 2.815 & 0.00345 \\
$(\mathrm{Nm})$ & 50\%ile Male & 0.90 & 0.027 & 16.636 & 3.734 & 0.00366 \\
& 95\%ile Male & 0.90 & 0.454 & 21.028 & 4.962 & 0.00401 \\
\hline 5\%ile Female & 0.85 & -0.012 & 0.372 & 0.106 & 0.00010 \\
\%MVC & 50\%ile Female & 0.84 & -0.009 & 0.441 & 0.131 & 0.00011 \\
95) & 5\%ile Female & 0.83 & 0.003 & 0.559 & 0.172 & 0.00012 \\
& 50\%ile Male & 0.83 & 0.001 & 0.270 & 0.089 & 0.00006 \\
& 95\%ile Male & 0.83 & 0.008 & 0.341 & 0.117 & 0.00006 \\
\hline
\end{tabular}




\section{Appendix D. Example of Training function scoring.}

The following shows the scoring approach for the Training function. A summary scoring checklist precedes the rubrics for the 15 associated HF elements. Elements with an 'N/A' label in the checklist denotes those elements deemed not applicable in this iteration of tool development. 


\section{Training Scoring Checklist}

\begin{tabular}{|c|c|c|c|}
\hline Human Factors Element & Score & Human Factors Element & Score \\
\hline $\begin{array}{l}\text { HF for performance not only injury } \\
\text { prevention }\end{array}$ & & Continuous review of process & \\
\hline $\begin{array}{l}\text { Cost of injuries/problem related to } \\
\text { source }\end{array}$ & N/A & Review as early as possible & N/A \\
\hline $\begin{array}{l}\text { Total HF cost considered } \\
\text { (direct and indirect) }\end{array}$ & & Multiple people input & N/A \\
\hline $\begin{array}{l}\text { Application reason (e.g. legislated or } \\
\text { culture) }\end{array}$ & & Level of subject matter expert need & \\
\hline Justification for change & & HF two-way communication & N/A \\
\hline HF Guidelines & & Reactive results tracking & N/A \\
\hline HF specific training & & $\begin{array}{l}\text { Lessons learned are logged and acted } \\
\text { upon }\end{array}$ & N/A \\
\hline HF training timeline and repetition & & Feed forward of information & N/A \\
\hline Employee development & & HF relevant metrics & \\
\hline $\begin{array}{l}\text { HF in experience delivery (employee's } \\
\text { work deliverables) }\end{array}$ & & $\begin{array}{l}\text { HF connection to traditional metrics } \\
\text { understood }\end{array}$ & N/A \\
\hline $\begin{array}{l}\text { HF includes physical and psychosocial } \\
\text { (employee's workplace) }\end{array}$ & & Strategy integration & \\
\hline $\begin{array}{l}\text { Control and adjustability of work } \\
\text { environment }\end{array}$ & N/A & HF culture & \\
\hline Maintenance for HF problem prevention & N/A & $\mathrm{HF}$ as value promotion & N/A \\
\hline $\begin{array}{l}\text { Feedback questionnaires that investigate } \\
\text { HF }\end{array}$ & & Celebrated - project promotion and ideal & N/A \\
\hline $\begin{array}{l}\text { HF review process } \\
\text { (for HF specific improvement) }\end{array}$ & N/A & $\begin{array}{l}\text { HF considerate hiring and development } \\
\text { package }\end{array}$ & N/A \\
\hline $\begin{array}{l}\text { HF review process applied } \\
\text { (outcome measure) }\end{array}$ & N/A & & \\
\hline
\end{tabular}

Grand Total

\% World Class (100* Grand Total / 64) 
HF for performance not only injury prevention (\#1):

\begin{tabular}{l|c}
\hline Level of Human Factors Maturity & Score \\
\hline No consideration of HF for performance in the function of Training - only related to injury & 0 \\
\hline HF rarely considered for performance in the function of Training & 1 \\
\hline HF occasionally considered for performance in the function of Training & 2 \\
\hline HF frequently considered for performance in the function of Training & 3 \\
\hline $\begin{array}{l}\text { HF considerations in the function of Training always considered for maximising individual } \\
\text { performance and minimising injury risk }\end{array}$ & 4 \\
\hline
\end{tabular}

Comments:

Total HF cost considered (direct and indirect) (\#3):

\begin{tabular}{l|c}
\hline Level of Human Factors Maturity & Score \\
\hline HF consideration in Training does not include cost of poor HF & 0 \\
\hline HF consideration in Training occasionally includes direct costs(injury related) of HF & 1 \\
No consideration of indirect costs & 2 \\
\hline HF consideration in Training always includes direct costs. & \\
No consideration of indirect costs. & 3 \\
\hline HF consideration in Training occasionally includes indirect costs and always includes direct \\
costs
\end{tabular}

Comments:

\begin{tabular}{l|c} 
Application reason (e.g. legislated or culture) (\#4): & Score \\
\hline Level of Human Factors Maturity & 0 \\
\hline No HF applied. & 1 \\
\hline HF should only be applied based on legislative requirements. & 2 \\
\hline HF should only be applied solely for injury prevention. & 3 \\
\hline HF applied for injury prevention and occasionally performance improvement. & 4 \\
\hline HF applied as part of culture of organization - maximize employee performance and well- \\
being.
\end{tabular}

Comments: 
Justification for change (\#5):

\begin{tabular}{l|c}
\hline Level of Human Factors Maturity & Score \\
\hline HF justification for change occurring within Training is at a level required by legislation. & \\
HF justification for change as promoted within Training content is at a level required by & 0 \\
legislation. & \\
\hline HF justification for change occurring within Training is at a level that occasionally exceeds & \\
legislation requirements (injury risk). & \\
HF justification for change promoted within Training is at a level that occasionally exceeds & \\
legislation requirements (injury risk). & \\
\hline HF justification for change occurring within Training is at a level that frequently exceeds \\
legislation requirements and considers human performance. \\
HF justification for change promoted within Training is at a level that frequently exceeds \\
legislation requirements and considers human performance. \\
\hline HF justification for change occurring within Training is at a level that always exceeds \\
legislation requirements and occasionally includes cost-benefit/return on investment. \\
HF justification for change promoted within Training is at a level that always exceeds \\
legislation requirements and occasionally includes cost-benefit/return on investment. \\
\hline HF justification for change occurring within Training is at a level that always exceeds \\
legislation requirements and non-injury, cost-benefit/return on investment. \\
HF justification for change promoted within Training is at a level that always exceeds \\
legislation requirements and non-injury, cost-benefit/return on investment. \\
\hline
\end{tabular}

Comments:

HF Guidelines (\#6):

\begin{tabular}{l|c}
\hline Level of Human Factors Maturity & Score \\
\hline HF training does not include HF guidelines. & 0 \\
\hline HF training occasionally includes generic HF guidelines. & 1 \\
\hline HF training frequently includes generic HF guidelines. & 2 \\
\hline HF training occasionally includes organization specific HF guidelines. & 3 \\
\hline HF training frequently includes organization specific HF guidelines. & 4 \\
\hline
\end{tabular}

Comments:

HF specific training (\#7):

\begin{tabular}{l|c}
\hline Level of Human Factors Maturity & Score \\
\hline No component of training considers HF. & 0 \\
\hline Generic HF component exists within health and safety. & 1 \\
\hline Designated HF component to training that is injury focused. & 3 \\
\hline $\begin{array}{l}\text { HF training component includes performance and well-being and content has one of } \\
\text { organization or function specific examples. }\end{array}$ & 4 \\
\hline $\begin{array}{l}\text { HF training component includes performance and well-being and content has organization } \\
\text { and multiple function specific examples. }\end{array}$ & \\
\hline
\end{tabular}

Comments: 
HF training timeline and repetition (\#8):

\begin{tabular}{l|c}
\hline Level of Human Factors Maturity & Score \\
\hline No HF related training completed. & 0 \\
\hline HF related training completed after first year. & 1 \\
HF training is rarely refreshed. & 2 \\
\hline HF training is completed within first year. & 3 \\
HF training is occasionally refreshed. & 3 \\
\hline HF training is completed prior to first required use. & 4 \\
HF training is refreshed every other year. & 4 \\
\hline HF training is completed as part of introductory training. &
\end{tabular}

Comments:

\section{Employee Development (\#9):}

\begin{tabular}{|c|c|}
\hline Level of Human Factors Maturity & Score \\
\hline No technical and personnel development or space for creativity. & 0 \\
\hline $\begin{array}{l}\text { Employee development rarely extends beyond personal work focus and rarely improves } \\
\text { capability and understanding in other aspects of work and life. } \\
\text { Creative space allows employee to think beyond prescribed work for continuous } \\
\text { improvement. }\end{array}$ & 1 \\
\hline $\begin{array}{l}\text { Employee development occasionally extends beyond personal work focus and occasionally } \\
\text { improves capability and understanding in other aspects of work and life. } \\
\text { Creative space allows employee to think beyond prescribed work for continuous } \\
\text { improvement. }\end{array}$ & 2 \\
\hline $\begin{array}{l}\text { Employee development frequently extends beyond personal work focus and frequently } \\
\text { improves capability and understanding in other aspects of work and life. } \\
\text { Creative space allows employee to think beyond prescribed work for continuous } \\
\text { improvement. }\end{array}$ & 3 \\
\hline $\begin{array}{l}\text { Employee development always extends beyond personal work focus and always improves } \\
\text { capability and understanding in other aspects of work and life. } \\
\text { Creative space allows employee to think beyond prescribed work for continuous } \\
\text { improvement. }\end{array}$ & 4 \\
\hline
\end{tabular}

Comments:

HF in experience delivery (employee's work deliverables) (\#10):

\begin{tabular}{l|c}
\hline Level of Human Factors Maturity & Score \\
\hline Employee's work has no consideration of internal/external customer experience. & 0 \\
\hline Employee's work rarely considers internal/external customer experience. & 1 \\
\hline Employee's work occasionally considers internal/external customer experience. & 2 \\
\hline Employee's work frequently considers internal/external customer experience. & 3 \\
\hline Employee's work always considers internal/external customer experience. & 4 \\
\hline
\end{tabular}

Comments: 
HF includes physical and psychosocial (employee's workplace) (\#11)

\begin{tabular}{l|c}
\hline Level of Human Factors Maturity & Score \\
\hline $\begin{array}{l}\text { HF consideration for the employee includes only some physical aspects and no psychosocial } \\
\text { considerations. }\end{array}$ & 0 \\
\hline $\begin{array}{l}\text { HF consideration for the employee includes many physical aspects and few psychosocial } \\
\text { considerations. }\end{array}$ & 1 \\
\hline $\begin{array}{l}\text { HF consideration for the employee includes physical and psychosocial aspects with } \\
\text { psychosocial findings considered of little value. }\end{array}$ & 2 \\
\hline $\begin{array}{l}\text { HF consideration for the employee includes physical and psychosocial aspects with } \\
\text { psychosocial considerations considered significant in majority of cases. }\end{array}$ & 3 \\
\hline $\begin{array}{l}\text { HF consideration for the employee includes physical and psychosocial considerations } \\
\text { equally. }\end{array}$ & 4 \\
\hline
\end{tabular}

Comments:

Feedback questionnaires that investigate HF (\#14):

\begin{tabular}{l|c}
\hline Level of Human Factors Maturity & Score \\
\hline No HF questionnaires review HF training content, procedures and outcomes. & 0 \\
\hline Questionnaires rarely review HF training content, procedures and outcomes. & 1 \\
\hline Questionnaires occasionally review HF training content, procedures and outcomes. & 2 \\
\hline Questionnaires frequently review HF training content, procedures and outcomes. & 3 \\
\hline Questionnaires always review HF training content, procedures and outcomes. & 4 \\
\hline
\end{tabular}

Comments:

Continuous review of process (\#17):

\begin{tabular}{l|c}
\hline Level of Human Factors Maturity & Score \\
\hline HF component of training is established and not reviewed. & 0 \\
\hline HF component of training is rarely reviewed for improvement. & 1 \\
\hline HF component of training is occasionally reviewed for improvement. & 2 \\
\hline HF component of training is frequently reviewed for improvement. & 3 \\
\hline HF component of training is always reviewed for improvement. & 4 \\
\hline
\end{tabular}

Comments:

Level of subject matter expert need (\#20):

\begin{tabular}{l|c}
\hline Level of Human Factors Maturity & Score \\
\hline HF component of training is completed by external subject matter expert. & 0 \\
\hline $\begin{array}{l}\text { HF component of training is completed jointly by external and internal subject matter } \\
\text { expert. }\end{array}$ & 1 \\
\hline $\begin{array}{l}\text { HF component of training is completed primarily by internal subject matter expert with } \\
\text { external expert recruited as required. }\end{array}$ & 2 \\
\hline HF component of training completed by internal subject matter expert. & 3 \\
\hline HF component of training facilitated by internal subject matter expert as needed. & 4 \\
\hline
\end{tabular}

Comments: 
HF relevant metrics (\#25):

\begin{tabular}{l|c}
\hline Level of Human Factors Maturity & Score \\
\hline No HF relevant metrics exist in Training. & 0 \\
\hline HF Training completion is tracked and not presented. & 1 \\
\hline HF Training completion is tracked and is presented. & 2 \\
\hline HF Training completion rate is tracked and not presented. & 3 \\
HF Training completed is tracked by organization function and not presented. & \\
\hline HF Training completion rate is tracked and is presented. & 4 \\
HF Training completed is tracked by organization function and is presented.
\end{tabular}

Comments:

Strategy integration (\#27):

\begin{tabular}{l|c}
\hline Level of Human Factors Maturity & Score \\
\hline No connection of HF to organization strategy. & \\
No communicated connection of HF to organization strategy. & 0 \\
No HF training connects HF to strategy. & 1 \\
\hline HF rarely connected to organization strategy. & \\
Communications occasionally connects HF to organization strategy. & \\
HF training occasionally connects HF to organization strategy. & 2 \\
\hline HF occasionally connected to organization strategy. & \\
Communications frequently connects HF to organization strategy. & \\
HF training frequently connects HF to organization strategy. & 3 \\
\hline HF frequently connected to organization strategy. & \\
Communications connects HF in strategy decisions to a generic organization outcome. \\
HF training always connects HF to organization strategy with examples of strategy decisions \\
to generic organization outcomes.
\end{tabular}

Comments: 
HF culture (\#28):

\begin{tabular}{|c|c|}
\hline Level of Human Factors Maturity & Score \\
\hline $\begin{array}{l}\text { HF impact in Training is unknown and not applied. } \\
\text { HF of suppliers and external partners or contractors are not considered important or } \\
\text { relevant. }\end{array}$ & 0 \\
\hline $\begin{array}{l}\text { HF impact in Training is understood by few, rarely applied, and only when mandated. } \\
\text { HF of suppliers and external partners or contractors are rarely considered important or } \\
\text { relevant. }\end{array}$ & 1 \\
\hline $\begin{array}{l}\text { HF impact in Training is understood by some, moderately applied, and only when mandated. } \\
\text { HF of suppliers and external partners or contractors are occasionally considered important } \\
\text { or relevant. }\end{array}$ & 2 \\
\hline $\begin{array}{l}\text { HF impact in Training is understood by most and frequently applied when mandated. } \\
\text { HF of suppliers and external partners or contractors are frequently considered important or } \\
\text { relevant. }\end{array}$ & 3 \\
\hline $\begin{array}{l}\text { HF impact in Training is understood by all and applied without being mandated. } \\
\text { HF of suppliers and external partners or contractors are always considered important or } \\
\text { relevant. }\end{array}$ & 4 \\
\hline
\end{tabular}

\section{Comments:}




\section{References}

Abras, C., Maloney-Krichmar, D., and Preece, J. (2004) 'User-centered design', in Bainbridge, W.S. (Ed.), Berkshire encyclopedia of human-computer interaction, Berkshire Publishing Company, Limited, Great Barrington, Massachusetts, pp. 763-768.

Allen, D.G., Lamb, G.D., and Westerblad, H. (2008) Skeletal muscle fatigue: cellular mechanisms. Physiological Review, 88(1), 287-332. doi: 10.1152/physrev.00015.2007.Repeated

American Cancer Society 'Economic impact of cancer' [online] http://www.cancer.org/cancer/cancerbasics/economic-impact-of-cancer (Accessed July 18 2013).

American Congress of Governmental Industrial Hygienists (2001) Threshold limit values and biological exposure indices for 2001. ACGIH, Cincinnati, OH.

Andersson, R., Eriksson, H., and Torstensson, H. (2006) Similarities and differences between TQM, six sigma and lean. The TQM Magazine, 18(3), 282-296. doi: $10.1108 / 09544780610660004$

Belbin, R.M. (1955) Older people and heavy work. British Journal of Industrial Medicine, 12(4), 309-319.

Ben-Gal, I., and Bukchin, J. (2002) The ergonomic design of workstations using virtual manufacturing and response surface methodology. IIE Transactions, 34(4), 375-391. doi: $10.1080 / 07408170208928877$

Bernard, M.D. (1997) Musculoskeletal disorders and workplace factors: A critical review of the epidemiologic evidence for work-related musculoskeletal disorders of the neck, upper extremity, and low back. US Department of Health and Human Services, National Institute for Occupational Safety and Health, Cinncinnati, $\mathrm{OH}$.

Bierwirth, M., Bruder, R., and Schaub, K. (2010) 'Total Ergonomics Management', Advances in Human Factors, Ergonomics, and Safety in Manufacturing and Service Industries, CRC Press, pp. 108-118.

Bigelow, P., and Robson, L., Occupational Health and Safety Management Audit Instruments: A Literature Review. (2005). Toront. (Access 2005)

Bititci, U.S., Carrie, A.S., and McDevitt, L. (1997) Integrated performance measurement systems: a development guide. International Journal of Operations \& Production Management, 17(5), 522-534.

Bititci, U.S., Mendibil, K., Nudurupati, S., Garengo, P., and Turner, T. (2006) Dynamics of performance measurement and organisational culture. International Journal of Operations \& Production Management, 26(12), 1325-1350. doi: 10.1108/01443570610710579

Bititci, U.S., Turner, T., and Begemann, C. (2000) Dynamics of performance measurement systems. International Journal of Operations \& Production Management, 20(6), 692704.

Bosch, T., Mathiassen, S.E., Visser, B., de Looze, M.P., and van Dieen, J.H. (2011) The effect of work pace on workload, motor variability and fatigue during simulated light assembly work. Ergonomics, 54(2), 154-68. doi: 10.1080/00140139.2010.538723 
Bourne, M., Mills, J., Neely, A., Platts, K., Wilcox, M., Hamblin, D., and Bicheno, J. (1999) Performance measurement system design: testing a process approach in manufacturing companies. International Journal of Business Performance Management, 1(2), 154-170.

Bourne, M., Mills, J., Wilcox, M., Neely, A., and Platts, K. (2000) Designing, implementing and updating performance measurement systems. International Journal of Operations \& Production Management, 20(7), 754-771.

Bourne, M., Neely, A., Mills, J., and Platts, K. (2003) Implementing performance measurement systems: a literature review. International Journal of Business Performance Management, 5(1).

Bourne, M., Neely, A., Platts, K., and Mills, J. (2002) The success and failure of performance measurement initiatives. International Journal of Operations \& Production Management, 22(11), 1288-1310. doi: 10.1108/01443570210450329

Braun, W.J., Rebollar, R., and Schiller, E.F. (1996) Computer aided planning and design of manual assembly systems. International Journal of Production Research, 34(8), 23172333.

Broberg, O. (2007) Integrationg ergonomics into engineering: empirical evidence and implications for the ergonomists. Human Factors and Ergonomics in Manufacturing, 17(4), 353-366. doi: 10.1002/hfm

Brown, M.G. (1996) Keeping Score: Using the Right Metrics to Drive World-Class Performance, Quality Resources, New York, NY.

Brown, M.G. (2007) Beyond the balanced scorecard: improving business intelligence with analytics, Productivity Press, New York.

Bucheim, R.K. (2000) Developing performance metrics for a design engineering department. IEEE Transactions on Engineering Management, 47(3), 309-320.

Buckle, P. (2011) 'The perfect is the enemy of the good' - ergonomics research and practice. Institute of Ergonomics and Human Factors Annual Lecture 2010. Ergonomics, 54(1), 111. doi: 10.1080/00140139.2010.542251

Canadian Standards Association (2012):2012: CSA Z1004, - Workplace ergonomics - A management and implementation Standard, Canadian Standards Association, Mississauga, Canada.

Canadian Standards Association Group 'How standards are developed' [online] http://www.csagroup.org/global/en/services/codes-and-standards/standardsdevelopment/how-standards-are-developed (Accessed July 28, 2015).

Caragnano, G., and Lavatelli, I. (2012) ERGO-MTM model: an integrated approach to set working times based upon standardized working performance and controlled biomechanical load. Work, 41(Suppl 1), 4422-4427. doi: 10.3233/WOR-2012-0740-4422

Carnahan, B.J., Norman, B.A., and Redfern, M.S. (2001) Incorporating physical demand criteria into assembly line balancing. IIE Transactions, 33(10), 875-887. doi: 10.1080/07408170108936880

Chaffin, D.B., Andersson, G.B.J., and Martin, B.J. (2006) Occupational Biomechanics, 4th ed., Wiley-Interscience.

Chapanis, A. (1995) Ergonomics in product development: a personal view. Ergonomics, 38(8), $1625-1638$.

Cherns, A. (1976) The principles of sociotechnical design. Human Relations, 29(8), 783-792.

Clegg, C.W. (2000) Sociotechnical principles for system design. Applied Ergonomics, 31(5), 463-477. 
Cole, D.C., Theberge, N., Dixon, S.M., Rivilis, I.R., Neumann, W.P., and Wells, R. (2009) Reflecting on a program of participatory ergonomics interventions: A multiple case study. Work, 34, 161-178. doi: 10.3233/WOR-2009-0914

Cross, K.F., and Lynch, R.L. (1988) The "SMART" way to define and sustain success. National Productivity Review, 8(1).

David, G.C. (2005) Ergonomic methods for assessing exposure to risk factors for work-related musculoskeletal disorders. Occupational Medicine (Oxford, England), 55(3), 190-199. doi: 10.1093/occmed/kqi082

Davis, F.D. (1989) Perceived usefulness, perceived ease of use, and user acceptance of information technology. MIS Quarterly, 13(3), 319-340.

Davis, F.D., Bagozzi, R.P., and Warshaw, P.R. (1989) User acceptance of computer technology: A comparison of two theoretical models. Management Science, 35(8), 982-1003.

de Looze, M.P., Vink, P., Koningsveld, E.A.P., Kuijt-Evers, L., and Van Rhijn, J.W. (2010) Cost-effectiveness of ergonomic interventions in production. Human Factors and Ergonomics in Manufacturing \& Service Industries, 20(4), 316-323. doi: 10.1002/hfm.20223

Deeney, C., and O'Sullivan, L. (2009) Work related psychosocial risks and musculoskeletal disorders: Potential risk factors, causation and evaluation methods. Work, 34(2), 239-248. doi: 10.3233/WOR-2009-0821

Dempsey, P.G., McGorry, R.W., and Maynard, W.S. (2005) A survey of tools and methods used by certified professional ergonomists. Applied Ergonomics, 36(4), 489-503. doi: 10.1016/j.apergo.2005.01.007

Diffrient, N., Tilley, A.R., Bardagjy, J.C., and Harman, D. (1973) Humanscale, MIT Press Cambridge, Massachusetts.

Dode, P. (2012) The Integration of Human Factors into Discrete Event Simulation and Technology Acceptance in Engineering Design. Unpublished MASc thesis, Ryerson University, Toronto, ON, Canada.

Doran, G.T. (1981) There's a S.M.A.R.T. way to write management's goals and objectives. Management Review, 70(11), 35-36.

Dreyfuss, H. (1966) The Measure of Man: Human Factors in Design, 2nd ed., Whitney Library of Design, New York.

Driessen, M.T., Groenewoud, K., Proper, K.I., Anema, J.R., Bongers, P.M., and van der Beek, A.J. (2010) What are possible barriers and facilitators to implementation of a Participatory Ergonomics programme? Implement Sci, 5, 64. doi: 10.1186/1748-5908-564

Drury, C.G. (2000) Human factors and quality: integration and new directions. Human Factors and Ergonomics in Manufacturing, 10(1), 45-59.

Dul, J., Bruder, R., Buckle, P., Carayon, P., Falzon, P., Marras, W.S., Wilson, J.R., and van der Doelen, B. (2012) A strategy for human factors/ergonomics: developing the discipline and profession. Ergonomics, 55(4), 377-395. doi: 10.1080/00140139.2012.661087

Dul, J., and Neumann, W.P. (2009) Ergonomics contributions to company strategies. Applied Ergonomics, 40(4), 745-752. doi: 10.1016/j.apergo.2008.07.001

Dym, C.L., Agogino, A.M., Eris, O., Frey, D.D., and Leifer, L.J. (2005) Engineering design thinking, teaching, and learning. Journal of Engineering Education, 94(1), 103-120.

Eason, K.D. (1995) User-centred design: for users or by users? Ergonomics, 38(8), 1667-1673. 
Edwards, K., and Jensen, P.L. (2014) Design of systems for productivity and well being. Applied Ergonomics, 45(1), 26-32. doi: http://dx.doi.org/10.1016/j.apergo.2013.03.022

Eklund, J.A.E. (1995) Relationships between ergonomics and quality in assembly work. Applied Ergonomics, 26(1), 15-20.

El ahrache, K., and Imbeau, D. (2009) Comparison of rest allowance models for static muscular work. International Journal of Industrial Ergonomics, 39(1), 73-80. doi: 10.1016/j.ergon.2008.10.012

El ahrache, K., Imbeau, D., and Farbos, B. (2006) Percentile values for determining maximum endurance times for static muscular work. International Journal of Industrial Ergonomics, 36(2), 99-108. doi: 10.1016/j.ergon.2005.08.003

Elkington, J. (1998) Cannibals with forks : the triple bottom line of 21st century business, New Society Publishers, Gabriola Island, BC.

Epstein, M.J., and Westbrook, R.A. (2001) Linking actions to profits in strategic decision making. MIT Sloan Management Review, 42(3), 39-49.

Erdinc, O., and Vayvay, O. (2008a) Ergonomics interventions improve quality in manufacturing: a case study. International Journal of Industrial and Systems Engineering, 3(6), 727-745.

Erdinc, O., and Vayvay, O. (2008b) Quality improvement through ergonomics methodology: conceptual framework and an application. International Journal of Productivity and Quality Management, 3(3), 311-324.

European Foundation for Quality Management 'The EFQM Excellence Model' [online] http://www.efqm.org/en/tabid/132/default.aspx (Accessed August 4, 2013 2013).

European Foundation for Quality Management 'Our history' [online] http://www.efqm.org/aboutus/our-history (Accessed July 28, 2015 2015).

Falck, A.-C., Örtengren, R., and Högberg, D. (2010) The impact of poor assembly ergonomics on product quality: A cost-benefit analysis in car manufacturing. Human Factors and Ergonomics in Manufacturing \& Service Industries, 20(1), 24-41. doi: 10.1002/hfm.20172

Fitzgerald, L., Johnston, R., Brignall, T.J., Silvestro, R., and Voss, C. (1991) Performance Measurement in Service Businesses, The Chartered Institute of Management Accountants, London.

Fitzgerald, L., and Moon, P. (1996) Performance Measurement in Service Industries: Making it Work, The Chartered Institute of Management Accountants, London.

Flapper, S.D.P., Fortuin, L., and Stoop, P.P.M. (1996) Towards consistent performance management systems. International Journal of Operations \& Production Management, 16(7), 27-37.

Franco, M., and Bourne, M. (2003) Factors that play a role in "managing through measures". Management Decision, 41(8), 698-710.

Freeman, R.E. (1994) The Politics of Stakeholder Theory: Some Future Directions. Business Ethics Quarterly, 4(4), 409-421. doi: 10.2307/3857340

Freeman, R.E., Wicks, A.C., and Parmar, B. (2004) Stakeholder Theory and "The Corporate Objective Revisited”. Organization Science, 15(3), 364-369. doi: 10.1287/orsc.1040.0066

Freivalds, A., and Niebel, B. (2009) Niebel's Methods, Standards, and Work Design, McGrawHill Higher Education, Boston.

Fritzsche, L., Wegge, J., Schmauder, M., Kliegel, M., and Schmidt, K.H. (2014) Good ergonomics and team diversity reduce absenteeism and errors in car manufacturing. Ergonomics. doi: 10.1080/00140139.2013.875597 
Fulton Suri, J. (2001) The Ergonomics Society--the Society Lectures 1999: the next 50 years: future challenges and opportunities for empathy in our science. Ergonomics, 44(14), 1278-89. doi: 10.1080/00140130110105850

Garde, A.H., Hansen, Å.M., and Jensen, B.R. (2003) Physiological responses to four hours of low-level repetitive work. Scandinavian Journal of Work, Environment \& Health, 29(6), 452-460. doi: 10.5271/sjweh.753

Garrety, K., and Badham, R. (1999) Trajectories, social worlds, and boundary objects: A framework for analyzing the politics of technology. Human Factors and Ergonomics in Manufacturing, 9(3), 277-290.

Garvin, D.A. (1991) How the Baldridge Award really works. Harvard Business Review, 69(6), 80-93.

Gilks, J., and Logan, R., Occupational injuries and diseases in Canada, 1996-2008: Injury rates and cost to the economy. (2010). Human Resources and Skills Development Canada, L.P., Occupational Health and Safety Division, Canada. (Access 2010)

Givi, Z.S., Jaber, M.Y., and Neumann, W.P. (2015) Production planning in DRC systems considering worker performance. Computers \& Industrial Engineering, 87, 317-327. doi: 10.1016/j.cie.2015.05.005

Godener, A., and Söderquist, K.E. (2004) Use and impact of performance measurement results in R\&D and NPD: an exploratory study. R\&D Management, 34(2), 191-219.

Goggins, R.W., Spielholz, P., and Nothstein, G.L. (2008) Estimating the effectiveness of ergonomics interventions through case studies: implications for predictive cost-benefit analysis. Journal of Safety Research, 39(3), 339-344. doi: 10.1016/j.jsr.2007.12.006

Gomes, C.F., Yasin, M.M., and Lisboa, J.V. (2004) A literature review of manufacturing performance measures and measurement in an organizational context: a framework and direction for future research. Journal of Manufacturing Technology Management, 15(6), 511-530. doi: 10.1108/17410380410547906

Gómez, J.G., Costa, M.M., and Lorente, Á.R.M. (2011) A critical evaluation of the EFQM model. International Journal of Quality \& Reliability Management, 28(5), 484-502. doi: $10.1108 / 02656711111132544$

Gosselin, M. (2005) An empirical study of performance measurement in manufacturing firms. International Journal of Productivity and Performance Management, 54(5/6), 419-437. doi: 10.1108/17410400510604566

Grachev, M., and Rakitsky, B. (2013) Historic horizons of Frederick Taylor's scientific management. Journal of Management History, 19(4), 512-527. doi: 10.1108/jmh-052012-0043

Greig, M., Village, J., and Neumann, W.P. (2013a) 'Predicting work load and efficiency during light assembly workstation design', Industrial and Systems Engineering Research Sessions (ISERC) at the IIE Annual Conference, San Juan, Peurto Rico.

Greig, M.A., Dixon, S.M., Annett, T.L., Village, J.L., and Neumann, W.P., (2013b), 'Usercentered development of a workstation efficiency evaluation tool' in Conference Usercentered development of a workstation efficiency evaluation tool.

Greig, M.A., and Neumann, W.P. (2015) Testing of a workstation efficiency evaluator tool. International Journal of Industrial Ergonomics, 48, 60-69. doi: 10.1016/j.ergon.2015.03.002

Greig, M.A., Patten, D., and Neumann, W.P., (2015), 'Quantifying Human Factors (HF) capabilities in an organisation - a macro-ergonomic tool for identifying new avenues for 
applying HF' in Conference Quantifying Human Factors (HF) capabilities in an organisation - a macro-ergonomic tool for identifying new avenues for applying HF.

Hackman, J.R., and Wageman, R. (1995) Total quality management: empirical, conceptual, and practical issues. Administrative Science Quarterly, 40(2), 309-342.

Hägg, G.M. (2003) Corporate initiatives in ergonomics — an introduction. Applied Ergonomics, 34(1), 3-15.

Haims, M.C., and Caryon, P. (1998) Theory and practice for the implemntation of 'in-hous', continuous improvement participatory ergonomic programs. Applied Ergonomics, 29(6), 461-472.

Haines, H., Wilson, J.R., Vink, P., and Koningsveld, E. (2002) Validating a framework for participatory ergonomics (the PEF). Ergonomics, 45(2), 309-327. doi: 10.1080/0014013021012351

Hall-Andersen, L.B., and Broberg, O. (2014) Integrating ergonomics into engineering design: The role of objects. Applied Ergonomics, 45(3), 647-654.

Hansson, G.-Å., Balogh, I., Ohlsson, K., Granqvist, L., Nordander, C., Arvidsson, I., Åkesson, I., Unge, J., Rittner, R., Strömberg, U., and Skerfving, S. (2010) Physical workload in various types of work: Part II. Neck, shoulder and upper arm. International Journal of Industrial Ergonomics, 40(3), 267-281. doi: 10.1016/j.ergon.2009.11.002

Hansson, G.Å., Arvidsson, I., Ohlsson, K., Nordander, C., Mathiassen, S.E., Skerfving, S., and Balogh, I. (2006) Precision of measurements of physical workload during standardised manual handling. Part II: Inclinometry of head, upper back, neck and upper arms. Journal of Electromyography and Kinesiology, 16(2), 125-136.

Hauser, J., and Katz, G. (1998) Metrics: You are what you measure! European Management Journal, 16(5), 12.

Heilala, J., Montonen, J., and Väätäinen, O. (2008) Life cycle and unit-cost analysis for modular reconfigurable flexible light assembly systems. Proceedings of the Institution of Mechanical Engineers, Part B: Journal of Engineering Manufacture, 222(10), 12891299. doi: $10.1243 / 09544054$ jem 1034

Helander, M.G. (1999) Seven common reasons to not implement ergonomics. International Journal of Industrial Ergonomics, 25(1), 97-101.

Helander, M.G. (2006) A Guide to Human Factors and Ergonomics, 2nd ed., Taylor \& Francis, Toronto.

Helander, M.G., and Burri, G.J. (1995) Cost effectiveness of ergonomics and quality improvements in electronics manufacturing. International Journal of Industrial Ergonomics, 15, 137-151.

Hendrick, H.W. (2003) Determining the cost-benefits of ergonomics projects and factors that lead to their success. Applied Ergonomics, 34(5), 419-427. doi: 10.1016/s00036870(03)00062-0

Hendrick, H.W., and Kleiner, B.M. (2001) Macroergonomics: An introduction to work system design., Human Factors and Ergonomics Society, Santa Monica, CA.

Hignett, S., and McAtamney, L. (2000) Rapid entire body assessment (REBA). Applied Ergonomics, 31(2), 201-205.

Hudson, M., Smart, A., and Bourne, M. (2001) Theory and practice in SME performance measurement systems. International Journal of Operations \& Production Management, 21(8), 1096-1115. 
Huwe, R.A. (2010) Metics 2.0: creating scorecards for high-performance teams and organizations, Praeger, Santa Barbara, California.

International Ergonomics Association 'The Discipline of Ergonomics' [online] http://www.iea.cc/whats/index.html (Accessed October 8, 2014 2014).

International Organization for Standardization (2002):2002: ISO 19011, Guidelines for quality and/or environmental management systems auditing, ISO, Geneva, Switzerland.

International Organization for Standardization (2010):2010: ISO/TR 7250-2:2010(E): Basic human body measurements for technological design - Part 2: Statistical summaries of body measurements from individual ISO populations, ISO, Geneva, Switzerland.

International Organization for Standardization 'Standards Development' [online] http://www.iso.org/iso/home/standards_development.htm (Accessed July 17 2013).

Jensen, P.L. (1997) Can participatory ergonomics become 'the way we do things in this firm'The Scandinavian approach to participatory ergonomics. Ergonomics, 40(10), 1078-1087. doi: 10.1080/001401397187612

Jensen, P.L. (2002) Human factors and ergonomics in the planning of production. International Journal of Industrial Ergonomics, 29(3), 121-131.

Johnson, H.T., and Kaplan, R.S. (1987) Relevance Lost: the Rise and Fall of Management Accounting, Harvard Business School Press, Boston, MA.

Kaghan, W.N., and Bowker, G.C. (2001) Out of machine age?: complexity, sociotechnical systems and actor network theory. Journal of Engineering and Technology Management, 18(3), 253-269.

Kaplan, R.S. (1984) The evolution of management accounting. The Accounting Review, 59(3), 390-418.

Kaplan, R.S. (1986) Accounting lag: the obsolescence of cost accounting systems. California Management Review, 28(2), 174-199.

Kaplan, R.S., and Norton, D.P. (1992) The balanced scorecard - measures that drive performance. Harvard Business Review, January-February, 71-79.

Kaplan, R.S., and Norton, D.P. (1996) The Balanced Scorecard : Translating Strategy Into Action, Harvard Business School Press, Boston, Mass.

Karasek, R., and Theorell, T. (1990) Healthy work : stress, productivity, and the reconstruction of working life, Basic Books, New York.

Karhu, O., Kansi, P., and Kuorinka, I. (1977) Correcting working postures in industry: a practical method. Applied Ergonomics, 8(4), 199-201.

Kazmierczak, K., Neumann, W.P., and Winkel, J. (2007) A case study of serial-flow car disassembly: Ergonomics, productivity and potential system performance. Human

Factors and Ergonomics in Manufacturing, 17(4), 331-351. doi: 10.1002/hfm.20078

Keegan, D.P., Eiler, R.G., and Jones, C.R. (1989) Are your performance measures obsolete? Management Accounting, 70(12), 45-50.

Keong Choong, K. (2013) Understanding the features of performance measurement system: a literature review. Measuring Business Excellence, 17(4), 102-121. doi: 10.1108/mbe-052012-0031

Kleiner, B.M. (1996) 'Macroergonomics lessons learned from large scale change efforts in industry, government, and academia', in Brown Jr, O. and Hendrick, H.W. (Eds.), Human Factrors in Organizational Design and Management - V, Amsterdam: North-Holland, pp. 483-487. 
Kleiner, B.M. (2004) Macroergonomics as a large work-system transformation technology. Human Factors and Ergonomics in Manufacturing, 14(2), 99-115.

Kleiner, B.M. (2006) Macroergonomics: analysis and design of work systems. Applied Ergonomics, 37(1), 81-9. doi: 10.1016/j.apergo.2005.07.006

Koski, A.V., and McGill, S.M. (1994) Dynamic shoulder flexion strength: for use in occupational risk analysis and clinical assessment. Clinical Biomechanics, 9(2), 99-104.

Kragt, H. (1995) Enhancing industrial performance: experiences of integrating the human factor. Ergonomics, 38(8), 1674-1685.

Kujala, S. (2003) User involvement: a review of the benefits and challenges. Behaviour \& Information Technology, 22(1), 1-16.

Lämkull, D., Hanson, L., and Roland, Ö. (2009) A comparative study of digital human modelling simulation results and their outcomes in reality: A case study within manual assembly of automobiles. International Journal of Industrial Ergonomics, 39(2), 428-441. doi: 10.1016/j.ergon.2008.10.005

Lannersten, L., Harms-Ringdahl, K., Schuldt, K., Ekholm, J., and Stockholm MUSIC 1 Study Group (1993) Isometric strength in flexors, abductors, and external rotators of the shoulder. Clinical Biomechanics, 8(5), 235-242.

Laring, J., Christmansson, M., Kadefors, R., and Örtengren, R. (2005) ErgoSAM: A preproduction risk identification tool. Human Factors and Ergonomics in Manufacturing, 15(3), 309-325. doi: 10.1002/hfm.20028

Larsman, P., and Hanse, J.J. (2009) The impact of decision latitude, psychological load and social support at work on the development of neck, shoulder and low back symptoms among female human service organization workers. International Journal of Industrial Ergonomics, 39(2), 442-446. doi: 10.1016/j.ergon.2009.01.002

Latham, G.P., and Locke, E.A. (2007) New developments in and directions for goal-setting research. European Psychologist, 12(4), 290-300.

Latham, G.P., and Yukl, G.A. (1975) A review of research on the application of goal setting in organizations. Academy of Management Journal, 18(4), 824-845.

Latko, W.A., Armstrong, T.J., Foulke, J.A., Herrin, G.D., Rabourn, R.A., and Ulin, S.S. (1997) Devleopment and evaluation of an observational method for assessing repetition in hand tasks. American Industrial Hygiene Association Journal, 58(4), 278-285.

Lee, K.S. (2005) Ergonomics in total quality management: how can we sell ergonomics to management? Ergonomics, 48(5), 547-58. doi: 10.1080/00140130400029282

Lee, S., and Koubek, R.J. (2012) Users' perceptions of usability and aesthetics as criteria of preand post-use preferences. European Journal of Industrial Engineering, 6(1), 87-117.

Leigh, J.P. (2011) Economic burden of occupational injury and illness in the United States. Millbank Quarterly, 89(4), 728-772.

Lewin, K. (1946) Action Research and Minority Problems. Journal of Social Issues, 2(4), 34-46. doi: 10.1111/j.1540-4560.1946.tb02295.x

Liker, J.K. (2004) The Toyota way : 14 management principles from the world's greatest manufacturer, McGraw-Hill, New York.

Lim, A.J., Village, J., Salustri, F.A., and Neumann, W.P. (2014) Process mapping as a tool for participative integration of human factors into work system design. European Journal of Industrial Engineering, 8(2), 273-290.

Lin, L., Drury, C.G., and Kim, S.-W. (2001) Ergonomics and quality in paced assembly lines. Human Factors and Ergonomics in Manufacturing, 11(4), 377-382. 
Lockett, J.F., Assmann, E., Green, R., Reed, M.P., Raschke, U., and Verriest, J.-P. (2005) 'Digital human modelling research and development user needs panel', Proceedings of the 2005 SAE Digital Human Modeling for Design and Engineering Symposium, Iowa City, USA.

Mankin, D., Cohen, S.G., and Sikson, T.K. (1997) Teams and technology: tensions in participatory design. Organizational Dynamics, 26(1), 63-76.

Mannonen, P., and Nieminen, M.P. (2006) 'User-centered product concept development', in Karwowski, W. (Ed.), International Encyclopedia of Ergonomics and Human Factors, CRC Press, Boca Raton, FL.

Marras, W.S., and Karwowski, W. (2006) Fundamentals and assessment tools for occupational ergonomics, CRC Press, Taylor \& Francis, Boca Raton, FL.

Mathiassen, S.E., and Åhsberg, E. (1999) Prediction of shoulder flexion endurance from personal factors. International Journal of Industrial Ergonomics, 24, 315-329.

McAtamney, L., and Corlett, N. (1993) RULA: a survey method for the investigation of workrelated upper limb disorders. Applied Ergonomics, 24(2), 91-99.

McClellan, A.J., Albert, W.J., Fischer, S.L., Seaman, F.A., and Callaghan, J.P. (2009) Estimating cumulative loads on the shoulder during automotive assembly tasks. Occupational Ergonomics, 8(2), 81-90. doi: 10.3233/oer-2009-0162

McCunn, P. (1998) The balanced scorecard: the eleventh commandment. Management Accounting, 76(11), 34-36.

McDowell, M.A., Fryar, C.D., Ogden, C.L., and Flegal, K.M. (2008) Anthropometric reference data for children and adults: United States, 2003-2006, US Department of Health and Human Services, Centers for Disease Control and Prevention, National Center for Health Statistics, Hyattsville, MD.

McTaggart, R. (1991) Principles for Participatory Action Research. Adult Education Quarterly, 41(3), 168-187. doi: 10.1177/0001848191041003003

Meerding, W.J., IJzelenberg, W., Koopmanschap, M.A., Severens, J.L., and Burdorf, A. (2005) Health problems lead to considerable productivity loss at work among workers with high physical load jobs. Journal of Clinical Epidemiology, 58(5), 517-523. doi: 10.1016/j.jclinepi.2004.06.016

Melnyk, S.A., Calantone, R.J., Luft, J., Stewart, D.M., Zsidisin, G.A., Hanson, J., and Burns, L. (2005) An empirical investigation of the metrics alignment process. International Journal of Productivity and Performance Management, 54(5/6), 312-324. doi: 10.1108/17410400510604494

Melnyk, S.A., Stewart, D.M., and Swink, M. (2004) Metrics and performance measurement in operations management: dealing with the metrics maze. Journal of Operations Management, 22, 209-217. doi: 10.1016/j.jom.2004.01.004

Mendibil, K., and MacBryde, J. (2006) Factors that affect the design and implementation of team-based performance measurement systems. International Journal of Productivity and Performance Management, 55(2), 118-142. doi: 10.1108/17410400610641708

Miles, B.L., and Swift, K. (1998) Design for manufacture and assembly. Manufacturing Engineer, 77(5), 221-224.

National Institute for Occupational Safety and Health (1994) Work practices guide for manual lifting. U.S. Department of Health and Human Services, Cincinnati, Ohio. 
National Institute of Standards and Technology (2011) '2011-2012 Criteria for Performance Excellence', Baldridge Performance Excellence Program, United States Department of Commerce, Gaithersburg, MD, USA.

National Institute of Standards and Technology 'Baldridge Performance Excellence Program' [online] http://www.nist.gov/baldrige/ (Accessed August 4, 2013 2013).

National Research Council (1999) Work-related Musculoskeletal Disorders: Report, Workshop Summary, and Workshop Papers, National Academy Press, Washington, D.C.

National Research Council (2001) Musculoskeletal Disorders and the Workplace - Low Back and Upper Extremities, National Academy Press, Washington DC.

Neely, A., Gregory, M., and Platts, K. (1995) Performance measurement system design: A literature review and research agenda. International Journal of Operations \& Production Management, 15(4), 80-116.

Neely, A., Mills, J., Platts, K., Richards, H., Gregory, M., Bourne, M., and Kennerley, M. (2000) Performance measurement system design: developing and testing a process-based approach. International Journal of Operations \& Production Management, 20(10), 11191145.

Neely, A.D., Adams, C., and Kennerley, M. (2002) The Performance Prism: The Scorecard for Measuring and Managing Business Success, FT Prenice Hall, London.

Neumann, W.P. (2004) Production Ergonomics: Identifying and Managing Risk in the Design of High Performance Work Systems. Doctoral Thesis, Lund University.

Neumann, W.P., Dixon, S.M., and Ekman, M. (2012) Ergonomics action research I: shifting from hypothesis testing to experiential learning. Ergonomics, 55(10), 1127-1139. doi: 10.1080/00140139.2012.700327

Neumann, W.P., and Dul, J. (2010) Human factors: spanning the gap between OM and HRM. International Journal of Operations \& Production Management, 30(9), 923-950. doi: 10.1108/01443571011075056

Neumann, W.P., Ekman, M., and Winkel, J. (2009) Integrating ergonomics into production system development--the Volvo Powertrain case. Applied Ergonomics, 40(3), 527-37. doi: 10.1016/j.apergo.2008.09.010

Neumann, W.P., Greig, M., Village, J., and Wells, R., (2013), 'Indicators for managing human centred manufacturing' in Conference Indicators for managing human centred manufacturing.

Neumann, W.P., Kihlberg, S., Medbo, P., Mathiassen, S.E., and Winkel, J. (2002) A case study evaluating the ergonomic and productivity impacts of partial automation strategies in the electronics industry. International Journal of Production Research, 40(16), 4059-4075. doi: 10.1080/00207540210148862

Neumann, W.P., and Village, J. (2012) Ergonomics action research II: a framework for integrating HF into work system design. Ergonomics, 55(10), 1140-1156. doi: 10.1080/00140139.2012.706714

Neumann, W.P., and Wells, R.P. (2007) 'Mechanical exposure assessment in the design of work', in Kumar, S. (Ed.), Biomechanics in Ergonomics 2nd ed., CRC Press, London.

Neumann, W.P., Wells, R.P., and Norman, R.W. (1999) '4DWATBAK: Adapting research tools and epidemiological findings to software for easy application by industrial personnel', Proceedings of the International Conference on Computer-Aided Ergonomics and Safety, Barcelona, Spain. 
Neumann, W.P., Winkel, J., Medbo, L., Magneberg, R., and Mathiassen, S.E. (2006) Production system design elements influencing productivity and ergonomics: A case study of parallel and serial flow strategies. International Journal of Operations \& Production Management, 26(8), 904-923. doi: 10.1108/01443570610678666

Norman, R., Wells, R., Neumann, P., Frank, J., Shannon, H., and Kerr, M. (1998) A comparison of peak vs cumulative physical work exposure risk factors for the reporting of low back pain in the automotive industry. Clinical Biomechanics, 13(8), 561-573.

Norman, W., and MacDonald, C. (2004) Getting to the bottom of "Triple Bottom Line". Business Ethics Quarterly, 14(2), 243-262.

Norros, L. (2014) Developing human factors/ergonomics as a design discipline. Applied Ergonomics, 45(1), 61-71. doi: 10.1016/j.apergo.2013.04.024

Occhipinti, E. (1998) OCRA: a concise index for the assessment of exposure to repetitive movements of the upper limbs. Ergonomics, 41(9), 1290-1311. doi: $10.1080 / 001401398186315$

Ottosson, S. (2003) Participation action research. Technovation, 23(2), 87-94. doi: 10.1016/s0166-4972(01)00097-9

Pascual, S.A., and Naqvi, S. (2008) An investigation of ergonomis analysis tools used in industry in the identification of work-related musculoskeletal disorders. International Journal of Safety and Ergonomics, 14(2), 237-245.

Pasmore, W. (2001) 'Action research in the workplace: the socio-technical perspective', in Reason, P. and Bradbury, H. (Eds.), Handbook of action research: participative inquiry \& practice, Sage Publications, London, pp. 38-47.

Peaucelle, J.L. (2000) From Taylorism to post-Taylorism. Journal of Organizational Change Management, 13(5), 452-467. doi: 10.1108/09534810010377426

Perez, J., and Neumann, W.P. (2015) Ergonomists' and engineers' views on the utility of virtual human factors tools. Human Factors and Ergonomics in Manufacturing \& Service Industries, 25(3), 279-293.

Perrow, C. (1983) The organizational context of human factors engineering. Administrative Science Quarterly, 28(4), 521-541.

Plagenhoef, S. (1971) Patterns of Human Motion, Prentice-Hall Inc., New Jersey.

Platts, K.W. (1994) Characteristics of methodologies for manufacturing strategy formulation. Computer Integrated Manufacutring Systems, 7(2), 93-99.

Punnett, L., Fine, L.J., Keyserling, W.M., Herrin, G.D., and Chaffin, D.B. (2000) Shoulder disorders and postural stress in automobile assembly work. Scandinavian Journal of Work, Environment \& Health, 26(4), 283-291. doi: 10.5271/sjweh.544

Putz-Anderson, V. (1988) Cumulative trauma disorders: a manual for musculoskeletal diseases of the upper limbs, Taylor \& Francis, London.

Robson, L.S., Macdonald, S., Gray, G.C., Van Eerd, D.L., and Bigelow, P.L. (2012) A descriptive study of the OHS management auditing methods used by public sector organizations conducting audits of workplaces: Implications for audit reliability and validity. Safety Science, 50(2), 181-189. doi: 10.1016/j.ssci.2011.08.006

Rodrigues, C.C. (1999) Ergonomic Tenets and the Bottom Line. Proceedings of the Human Factors and Ergonomics Society Annual Meeting, 43(14), 820-824. doi: $10.1177 / 154193129904301402$ 
Roethlisberger, F.J., and Dickson, W.J. (1966) Management and the worker : an account of a research program conducted by the Western Electric Company, Hawthorne Works, Chicago, Harvard University Press, Cambridge, Mass.

Rose, L., Ericson, M., Glimskär, B., Nordgren, B., and Örtengren, R., (1992), 'Ergo-index: development of a model to determine pause needs after fatigue and pain reactions during work' in Conference Ergo-index: development of a model to determine pause needs after fatigue and pain reactions during work, Elsevier Science Publishers B.V., Amsterdam, North-Holland, pp. 461-468.

Rose, L., Orrenius, U.E., and Neumann, W.P. (2013) Work environment and the bottom line: Survey of tools relating work environment to business results. Human Factors and Ergonomics in Manufacturing \& Service Industries, 23(5), 368-381. doi: 10.1002/hfm.20324

Rosecrance, J.C., and Cook, T.M. (2000) The use of participatory action research and ergonomics in the prevention of work-related musculoskeletal disorders in the newspaper industry. Applied Occupational and Environmental Hygiene, 15(3), 255-62. doi: 10.1080/104732200301575

Schaub, K., Caragnano, G., Britzke, B., and Bruder, R. (2013) The European Assembly Worksheet. Theoretical Issues in Ergonomics Science, 14(6), 616-639. doi: 10.1080/1463922x.2012.678283

Seaman, F.A., Albert, W.J., Weldon, N.R.E., Croll, J., and Callaghan, J.P. (2010) Biomechanical shoulder loads and postures in light automotive assembly workers: Comparison between shoulder pain/no pain groups. Work, 35(1), 39-48. doi: 10.3233/wor-2009-0956

Searcy, C., Dixon, S.M., and Neumann, W.P. (2016) The use of work environment performance indicators in corporate social responsibility reporting. Journal of Cleaner Production, 112, 2907-2921. doi: 10.1016/j.jclepro.2015.10.081

Simon, H.A. (1956) Raional choice and the structure of the environment. Psychological Review, 63(2), 129-138.

Simon, H.A. (1997) 'IV. Behavioral economics and bounded rationality', Models of Bounded Rationality: Emperically Grounded Economic Reason, Volume 3, The MIT Press, pp. 295-298.

Smith, M.E. (2003) Changing an organisation's culture: correlates of success and failure. Leadership \& Organization Development Journal, 24(5), 249-261.

So, R.H., and Lam, S.T. (2014) Factors affecting the appreciation generated through applying human factors/ergonomics (HFE) principles to systems of work. Applied Ergonomics, 45(1), 99-109. doi: 10.1016/j.apergo.2013.04.019

Stanton, N.A., and Stammers, R.B. (2008) Bartlett and the future of ergonomics. Ergonomics, 51(1), 1-13. doi: 10.1080/00140130701801116

Svendsen, S.W., Bonde, J.P., Mathiassen, S.E., Stengaard-Pedersen, K., and Frich, L.H. (2004) Work related shoulder disorders: quantitative exposure-response relations with reference to arm posture. Occupational and Environmental Medicine, 61(10), 844-853. doi: 10.1136/oem.2003.010637

Takala, E.P., Pehkonen, I., Forsman, M., Hansson, G.A., Mathiassen, S.E., Neumann, W.P., Sjøgaard, G., Veiersted, K.B., Westgaard, R.H., and Winkel, J. (2010) Systematic evaluation of observational methods assessing biomechanical exposures at work. Scandinavian Journal of Work, Environmental \& Health, 36(1), 3-24. 
Theberge, N., and Neumann, W.P. (2010) Doing 'organizational work': expanding the conception of professional practice in ergonomics. Applied Ergonomics, 42(1), 76-84. doi: 10.1016/j.apergo.2010.05.002

Theberge, N., and Neumann, W.P. (2013) The Relative Role of Safety and Productivity in Canadian Ergonomists' Professional Practices. Relations Industrielles/Industrial Relations, 68(3), 387-408.

Thomas, D.R. (2006) A General Inductive Approach for Analyzing Qualitative Evaluation Data. American Journal of Evaluation, 27(2), 237-246. doi: 10.1177/1098214005283748

Toccafondi, G., Albolino, S., Bellandi, T., and Venneri, F. (2012) Handover process: how to improve quality and safety through an ergonomic solution. Work, 41 Suppl 1, 2941-2945. doi: 10.3233/WOR-2012-0660-2941

Tompa, E., Culyer, A.J., and Dolinschi, R. (2008) Economic evaluation of interventions for occupational health and safety: developing good practice, Oxford University Press, Oxford.

Trochim, W.M.K., and Donnelly, J.P. (2007) Research methods knowledge base, Thomson Custom Publishing, Mason, $\mathrm{OH}$.

Turney, P.B.B., and Anderson, B. (1989) Accounting for continuous improvement. Sloan Management Review, 30(2), 37-47.

van Eerd, D., Cole, D., Irvin, E., Mahood, Q., Keown, K., Theberge, N., Village, J., St Vincent, M., and Cullen, K. (2010) Process and implementation of participatory ergonomic interventions: a systematic review. Ergonomics, 53(10), 1153-66. doi:

10.1080/00140139.2010.513452

Village, J., Greig, M., Salustri, F., Zolfaghari, S., and Neumann, W.P. (2014a) An ergonomics action research demonstration: integrating human factors into assembly design processes. Ergonomics, 57(10), 1574-89. doi: 10.1080/00140139.2014.938128

Village, J., Greig, M., Zolfaghari, S., Salustri, F., and Neumann, W.P. (2014b) Adapting Engineering Design Tools to Include Human Factors. IIE Transactions on Occupational Ergonomics and Human Factors, 2(1), 1-14. doi: 10.1080/21577323.2014.905884

Village, J., Salustri, F.A., and Neumann, W.P. (2013a) Cognitive mapping: Revealing the links between human factors and strategic goals in organizations. International Journal of Industrial Ergonomics, 43(4), 304-313. doi: 10.1016/j.ergon.2013.05.001

Village, J., Searcy, C., Salustri, F., and Patrick Neumann, W. (2015) Design for human factors (DfHF): a grounded theory for integrating human factors into production design processes. Ergonomics, 58(9), 1529-46. doi: 10.1080/00140139.2015.1022232

Village, J.L., Annett, T., Greig, M.A., and Neumann, W.P., (2013b), 'Ergonomics training for engineers: are we meeting their needs?' in Conference Ergonomics training for engineers: are we meeting their needs?

Vink, P., Koningsveld, E.A., and Molenbroek, J.F. (2006) Positive outcomes of participatory ergonomics in terms of greater comfort and higher productivity. Applied Ergonomics, 37(4), 537-46. doi: 10.1016/j.apergo.2006.04.012

Vogt, J., Leonhardt, J., Koper, B., and Pennig, S. (2010) Human factors in safety and business management. Ergonomics, 53(2), 149-63. doi: 10.1080/00140130903248801

Waters, T.R., Dick, R.B., and Krieg, E.F. (2011) Trends in work-related musculoskeletal disorders: a comparison of risk factors for symptoms using quality of work life data from the 2002 and 2006 general social survey. Journal of Occupational and Environmental Medicine, 53(9), 1013-24. doi: 10.1097/JOM.0b013e3181fc8493 
Waterson, P., and Kolose, S.L. (2010) Exploring the social and organisational aspects of human factors integration: A framework and case study. Safety Science, 48(4), 482-490. doi: 10.1016/j.ssci.2009.12.018

Wells, R., Mathiassen, S.E., Medbo, L., and Winkel, J. (2007) Time--a key issue for musculoskeletal health and manufacturing. Applied Ergonomics, 38(6), 733-44. doi: 10.1016/j.apergo.2006.12.003

Wells, R., Norman, R., Neumann, P., Andrews, D., Frank, J., Shannon, H., and Kerr, M. (1997) Assessment of physical work load in epidemiologic studies: common measurement metrics for exposure assessment. Ergonomics, 40(1), 51-61. doi: 10.1080/001401397188369

Wells, R.P., Neumann, W.P., Nagdee, T., and Theberge, N. (2013) Solution Building Versus Problem Convincing: Ergonomists Report on Conducting Workplace Assessments. IIE Transactions on Occupational Ergonomics and Human Factors, 1(1), 50-65. doi: 10.1080/21577323.2012.708699

Widanarko, B., Legg, S., Devereux, J., and Stevenson, M. (2014) The combined effect of physical, psychosocial/organisational and/or environmental risk factors on the presence of work-related musculoskeletal symptoms and its consequences. Applied Ergonomics, 45(6), 1610-21. doi: 10.1016/j.apergo.2014.05.018

Wilmore, J.H., and Costill, D.L. (1994) Physiology of sport and exercise, Human Kinetics Publisher, United States of America.

Wilson, J.R. (1994) Devolving ergonomics: the key to ergonomics managment programmes. Ergonomics, 37(4), 579-594.

Wilson, J.R. (2014) Fundamentals of systems ergonomics/human factors. Applied Ergonomics, 45(1), 5-13. doi: 10.1016/j.apergo.2013.03.021

Wren, D.A., and Greenwood, R.G. (1998) 'Motivators', Management innovators: the people and ideas that have shaped modern business, Oxford University Press, New York, pp. 171176.

Wulff, I.A., Westgaard, R.H., and Rasmussen, B. (1999a) Ergonomic criteria in large-scale engineering design - I Management by documentation only? Formal organization vs. designers' perceptions. Applied Ergonomics, 30(3), 191-205.

Wulff, I.A., Westgaard, R.H., and Rasmussen, B. (1999b) Ergonomic criteria in large-scale engineering design - II Evaluating and applying requirements in the real world of design. Applied Ergonomics, 30(3), 207-221.

Yeow, P.H.P., and Sen, R.N. (2003) Quality, productivity, occupational health and safety and cost effectiveness of ergonomic improvements in the test workstations of an electronic factory. International Journal of Industrial Ergonomics, 32(3), 147-163. doi: 10.1016/s0169-8141(03)00051-9

Yeow, P.H.P., and Sen, R.N. (2006) Productivity and quality improvements, revenue increment, and rejection cost reduction in the manual component insertion lines through the application of ergonomics. International Journal of Industrial Ergonomics, 36(4), 367377. doi: 10.1016/j.ergon.2005.12.008

Yin, R.K. (2009) Case study research: design and methods, 4th ed., Sage Publications, Inc., Los Angeles, California.

Zatsiorsky, V., and Seluyanov, V. (1983) 'The mass and inertia characteristics of main segments of human body', in Matsi, H. and Kobayashi, K. (Eds.), Biomechanics VIII-B, Human Kinetics Publishers, Champaign, Illinois, pp. 1152-1159. 
Zha, X.F. (2001) A neuro-fuzzy hybrid scheme for desgn and simulation of human machine systems. Applied Artificial Intelligence, 15(9), 797-823.

Zimmerman, B.J. (2008) 'Goal setting: a key proactive source of academic self-regulation', in Schunk, D.H. and Zimmerman, B.J. (Eds.), Motivation and self-regulated learning: theory, research, and applications, Lawrence Erlbaum Associates, New York, pp. 267295.

Zink, K.J., and Seibert, S. (2009) 'Performance Measurement from a Macroergonomics Perspective', in Schlick, C.M. (Ed.), Industrial Engineering and Ergonomics - Visions, Concepts, Methods and Tools, Springer-Verlag Berlin Heidelberg, Heidelberg, pp. 91103. 